\title{
Desingularizing isolated conical singularities: Uniform estimates via weighted Sobolev spaces
}

\author{
Tommaso Pacini
}

\begin{abstract}
We define a very general "parametric connect sum" construction that can be used to eliminate isolated conical singularities of Riemannian manifolds. We then show that various important analytic and elliptic estimates, formulated in terms of weighted Sobolev spaces, can be obtained independently of the parameters used in the construction. Specifically, we prove uniform estimates related to (i) Sobolev Embedding Theorems, (ii) the invertibility of the Laplace operator and (iii) Poincaré and Gagliardo-NirenbergSobolev-type inequalities.

Our main tools are the well-known theories of weighted Sobolev spaces and elliptic operators on "conifolds". We provide an overview of both, together with an extension of the former to general Riemannian manifolds.

For a geometric application of our results we refer the reader to our paper [15] concerning desingularizations of special Lagrangian conifolds in $\mathbb{C}^{m}$.
\end{abstract}

1 Introduction 106

2 Preliminaries 110

Part 1. Sobolev Embedding Theorems 113

3 Review of the theory of standard Sobolev spaces 113

4 Scaled Sobolev spaces 122

5 Weighted Sobolev spaces 128

6 Application: manifolds with ends modelled on cones and cylinders 
Part 2. Elliptic estimates

7 Fredholm results for elliptic operators on A.Cyl. manifolds

8 Weight-crossing 144

9 Fredholm results for elliptic operators on conifolds 146

10 Application: harmonic functions on conifolds 150

Part 3. Conifold connect sums and uniform estimates $\quad 155$

11 Conifold connect sums 155

12 The Laplacian on conifold connect sums 161

13 Further Sobolev-type inequalities on conifold connect sums

Acknowledgments

References

\section{Introduction}

It is a common problem in differential geometry to produce examples of (possibly immersed) Riemannian manifolds $(L, g)$ satisfying a given geometric constraint, usually a non-linear partial differential equation, on the metric (Einstein, constant scalar curvature, etc.) or on the immersion (constant mean curvature, minimal, etc.). If $L$ (or the immersion) happens to be singular, one then faces the problem of "desingularizing" it to produce a new, smooth, Riemannian manifold satisfying the same constraint. Often, one actually hopes to produce a family $\left(L_{t}, g_{t}\right)$ of manifolds satisfying the constraint and which converges in some sense to $(L, g)$ as $t \rightarrow 0$. One typical way to solve this problem is via "gluing". We outline this construction as follows, focusing for simplicity on the situation, where $L$ has only isolated point singularities and the constraint is on the metric.

Step 1: For each singular point $x \in L$, we look for an explicit smooth "local model": i.e., a manifold $(\hat{L}, \hat{g})$ which satisfies a related, scale-invariant, constraint and which, outside of some compact region, is topologically and 
metrically similar to an annulus $B\left(x, \epsilon_{1}\right) \backslash B\left(x, \epsilon_{2}\right)$ in $L$, centred in the singularity. We can then glue $\hat{L}$ onto the manifold $L \backslash B\left(x, \epsilon_{2}\right)$, using the "neck region" $B\left(x, \epsilon_{1}\right) \backslash B\left(x, \epsilon_{2}\right)$ to interpolate between the two metrics. The fact that the neck region is "small" is usually not a problem: one can simply rescale $\hat{g}$ to $t^{2} \hat{g}$, so that now $\left(\hat{L}, t^{2} \hat{g}\right)$ is of similar size. The resulting manifold, which we denote $(\hat{L} \# L, \hat{g} \# g)$, satisfies the constraints outside of the neck region simply by construction. If the interpolation is done carefully we also get very good control over what happens on the neck. We think of $(\hat{L} \# L, \hat{g} \# g)$ as an "approximate solution" to the gluing problem. Rescaling also gives a way to build families: the idea is to glue $\left(\hat{L}, t^{2} \hat{g}\right)$ into $B\left(x, \epsilon_{1}\right) \backslash B\left(x, t \epsilon_{2}\right)$, producing a family $\left(L_{t}, g_{t}\right)$; intuitively, as $t \rightarrow 0$ the compact region in $\hat{L}$ collapses to the singular point $x$ and $L_{t}$ converges to $L$.

Step 2: We now need to perturb each $\left(L_{t}, g_{t}\right)$ so that the resulting family satisfies the constraint globally. Thanks to a linearization process, the perturbation process often boils down to studying a linear elliptic system on $g_{t}$. One of the main problems is to verify that this system satisfies estimates which are uniform in $t$. This is the key for obtaining the desired perturbation for all sufficiently small $t$. Roughly speaking, there is often a delicate balance to be found as $t \rightarrow 0$ : on the one hand, if $L_{t}$ was built properly, as $t \rightarrow 0$ it will get closer to solving the constraint; on the other hand, it becomes more singular. Uniform estimates are important in proving that this balance can be reached.

The geometric problem defines the differential operator to be studied. However, this operator is often fairly intrinsic, and can be defined independently of the geometric specifics. The necessary estimates may likewise be of a much more general nature. Filtering out the geometric "super-structure" and concentrating on the analysis of the appropriate category of abstract Riemannian manifolds will then enhance the understanding of the problem, leading to improved results and clarity. The first goal of this paper is thus to set up an abstract framework for dealing with gluing constructions and the corresponding uniform estimates. Here, "abstract" means: independent of any specific geometric problem. We focus on gluing constructions concerning Riemannian manifolds with isolated conical singularities. These are perhaps the simplest singularities possible, but in the gluing literature they often appear as an interesting and important case. Our framework involves two steps, parallel to those outlined above.

Step A: In Section 11 we define a general connect sum construction between Riemannian manifolds, extrapolating from standard desingularization procedures. 
Step B: We show how to produce uniform estimates on these connect sum manifolds, by presenting a detailed analysis of three important Problems: (i) Sobolev Embedding Theorems, (ii) invertibility of the Laplace operator and (iii) Poincaré and Gagliardo-Nirenberg-Sobolev-type inequalities. The main results are Theorems 11.7, 12.2, 12.3, 13.1 and Corollary 13.2.

Our Step A is actually much more general than Step 1, as described above: it is specifically designed to deal with both compact and non-compact manifolds and it allows us to replace the given singularity not only with smooth compact regions but also with non-compact "asymptotically conical (AC) ends" or even with new singular regions. It also allows for different "neck-sizes" around each singularity. In this sense it offers a very broad and flexible framework to work with.

The range of possible estimates covered by our framework is clearly much wider than the set of Problems (i)-(iii) listed in Step B. Indeed, the underlying, well-known, theory of elliptic operators on conifolds is extremely general. Within this paper, this choice is to be intended as fairly arbitrary: among the many possible, we choose three estimates of general interest but differing one from the other in flavour: Problem (i) is of a mostly local nature; Problems (ii)-(iii) are global. In reality, however, our choice of Problems (i)-(iii) is based on the very specific geometric problems we happen to be interested in. The second goal of this paper is thus to lay down the analytic foundations for our papers $[14,15]$ concerning deformations and desingularizations of submanifolds whose immersion map satisfies the special Lagrangian constraint. The starting point for this work was a collection of gluing results concerning special Lagrangian submanifolds due to Arezzo-Pacard [2], Butscher [3], Lee [9] and Joyce [6, 7], and parallel results concerning coassociative submanifolds due to Lotay [12]. It slowly became apparent, thanks also to many conversations with some of these authors, that several parts of these papers could be simplified, improved or generalized: related work is currently still in progress. In particular, building approximate solutions and setting up the perturbation problem requires making several choices, which then influence the analysis rather drastically. A third goal of the paper is thus to present a set of choices which leads to very clean, simple and general results. One such choice concerns the parametrization of the approximate solutions: parametrizing the necks so that they depend explicitly on the parameter $t$ is one ingredient in obtaining uniform estimates. A second ingredient is the consistent use, even when dealing with compact manifolds, of weighted rather than 
standard Sobolev spaces. Although such choices may seem obvious to some members of the "gluing community", it still seems useful to emphasize this point.

For expository purposes we found it useful to split the paper into three separate parts. Part I is devoted to weighted Sobolev spaces and the corresponding Sobolev Embedding Theorems. The main example we are interested in is the case of "conifolds"; in this special case the Sobolev Embedding Theorems, cf. Corollary 6.8, are well-known. However, Problem (i) requires keeping close track of how the corresponding Sobolev constants depend on the conifolds and on the other data used in the connect sum construction. It is thus useful to step back and investigate exactly which properties of Sobolev spaces are crucial to the validity of Embedding Theorems. In the standard, i.e., non-weighted, case, the book by Hebey [4] provides an excellent introduction to this problem. Given the lack of an analogous reference for weighted Sobolev spaces, we devote a fair amount of attention to their definition and properties. Our main result in Part I is Theorem 5.1, which proves the validity of the Sobolev Embedding Theorems under fairly general hypotheses on the "scale" and "weight" functions with which we define these spaces.

Part II is devoted to the Fredholm theory of elliptic operators on conifolds. This theory is well-known but, for the reader's convenience, we review it (together with its asymptotically cylindrical counterpart) in Sections 7 and 9 . Sections 8 and 10 contain instead some useful consequences of the Fredholm theory.

Part III contains the main results of this paper, corresponding to Steps A and B, above: the definition of "conifold connect sums" and the uniform estimates, Problems (i)-(iii).

We conclude with one last comment. Depending on the details, the connect sum construction can have two outcomes: compact or non-compact manifolds. In the context of weighted spaces, Problem (i) does not note the difference. Problems (ii) and (iii) require instead that the kernels of the operators in question vanish. On non-compact manifolds this can be achieved very simply, via an a priori choice of weights: roughly speaking, we require that there exist non-compact "ends", then put weights on them which kill the kernel. This topological assumption is perfectly compatible with the geometric applications described in [15]. On compact manifolds it is instead necessary to work transversally to the kernel; uniform estimates depend on allowing the subspace itself to depend on the parameter $t$. We refer to Section 12 for details. 


\section{Preliminaries}

Let $(L, g)$ be an oriented $m$-dimensional Riemannian manifold. We can identify its tangent and cotangent bundles via the maps

(2.1) $T_{x} L \rightarrow T_{x}^{*} L, \quad v \mapsto v^{\#}:=g(v, \cdot), \quad$ with inverse $T_{x}^{*} L \rightarrow T_{x} L, \quad \alpha \mapsto \alpha^{b}$.

There are induced isomorphisms on all higher-order tensor bundles over $L$. In particular, the metric tensor $g$, as a section of $\left(T^{*} L\right)^{2}$, corresponds to a tensor $g^{b}$, section of $(T L)^{2}$. This tensor defines a natural metric on $T^{*} L$ with respect to which the map of Equation (2.1) is an isometry. In local coordinates, if $g=g_{i j} d x^{i} \otimes d x^{j}$ then $g^{b}=g^{i j} \partial_{i} \otimes \partial_{j}$, where $\left(g^{i j}\right)$ denotes the inverse matrix of $\left(g_{i j}\right)$.

Given any $x \in L$ we denote by $i_{x}(g)$ the injectivity radius at $x$, i.e., the radius of the largest ball in $T_{x} L$ on which the exponential map is a diffeomorphism. We then define the injectivity radius of $L$ to be the number $i(g):=\inf _{x \in L} i_{x}(g)$. We denote by $\operatorname{Ric}(g)$ the Ricci curvature tensor of $L$ : for each $x \in L$, this gives an element $\operatorname{Ric}_{x}(g) \in T_{x}^{*} L \otimes T_{x}^{*} L$.

Let $E$ be a vector bundle over $L$. We denote by $C^{\infty}(E)$ (respectively, $C_{c}^{\infty}(E)$ ) the corresponding space of smooth sections (respectively, with compact support). If $E$ is a metric bundle we can define the notion of a metric connection on $E$ : namely, a connection $\nabla$ satisfying

$$
\nabla(\sigma, \tau)=(\nabla \sigma, \tau)+(\sigma, \nabla \tau)
$$

where $(\cdot, \cdot)$ is the appropriate metric. We then say that $(E, \nabla)$ is a metric pair.

Recall that coupling the Levi-Civita connection on $T L$ with a given connection on $E$ produces induced connections on all tensor products of these bundles and of their duals. The induced connections depend linearly on the initial connections. Our notation will usually not distinguish between the initial connections and the induced connections: this is apparent when we write, for example, $\nabla^{2} \sigma$ (short for $\nabla \nabla \sigma$ ). Recall also that the difference between two connections $\nabla, \hat{\nabla}$ defines a tensor $A:=\nabla-\hat{\nabla}$. For example, if the connections are on $E$ then $A$ is a tensor in $T^{*} L \otimes E^{*} \otimes E$. Once again, we will not distinguish between this $A$ and the $A$ defined by any induced connections.

Let $E, F$ be vector bundles over $L$. Let $P: C^{\infty}(E) \rightarrow C^{\infty}(F)$ be a linear differential operator with smooth coefficients, of order $n$. We can then write $P=\sum_{i=0}^{n} A_{i} \cdot \nabla^{i}$, where $A_{i}$ is a global section of $(T L)^{i} \otimes E^{*} \otimes F$ and . 
denotes an appropriate contraction. Note that since $P$ is a local operator it is completely defined by its behaviour on compactly-supported sections.

Remark 2.1. Assume $P=\sum_{i=0}^{n} A_{i} \cdot \nabla^{i}$. Choose a second connection $\hat{\nabla}$ on $E$ and set $A:=\nabla-\hat{\nabla}$. Substituting $\nabla=\nabla-\hat{\nabla}+\hat{\nabla}=A+\hat{\nabla}$ allows us to write $P$ in terms of $\hat{\nabla}$. Note that the new coefficient tensors $\hat{A}_{i}$ will depend on $A$ and on its derivatives $\hat{\nabla}^{k} A$.

Now assume $E$ and $F$ are metric bundles. Then $P$ admits a formal adjoint $P^{*}: C^{\infty}(F) \rightarrow C^{\infty}(E)$, uniquely defined by imposing

$$
\int_{L}(P \sigma, \tau)_{F} \operatorname{vol}_{g}=\int_{L}\left(\sigma, P^{*} \tau\right)_{E} \operatorname{vol}_{g}, \quad \forall \sigma \in C_{c}^{\infty}(E), \tau \in C_{c}^{\infty}(F)
$$

$P^{*}$ is also a linear differential operator, of the same order as $P$.

Example 2.2. The operator $\nabla: C^{\infty}(E) \rightarrow C^{\infty}\left(T^{*} L \otimes E\right)$ has a formal adjoint $\nabla^{*}: C^{\infty}\left(T^{*} L \otimes E\right) \rightarrow C^{\infty}(E)$. Given $P=\sum_{i=0}^{n} A_{i} \cdot \nabla^{i}$, we can write $P^{*}$ in terms of $\nabla^{*}$. For example, choose a smooth vector field $X$ on $L$ and consider the operator $P:=\nabla_{X}=X \cdot \nabla: C^{\infty}(E) \rightarrow C^{\infty}(E)$. Then $\left(\nabla_{X}\right)^{*} \sigma=\nabla^{*}\left(X^{\#} \otimes \sigma\right)$.

The $\nabla$-Laplace operator on $E$ is defined as $\Delta:=\nabla^{*} \nabla: C^{\infty}(E) \rightarrow$ $C^{\infty}(E)$. When $E$ is the trivial $\mathbb{R}$-bundle over $L$ and we use the Levi-Civita connection, this coincides with the standard positive Laplace operator acting on functions

$$
\Delta_{g}:=-\operatorname{tr}_{g}\left(\nabla^{2}\right)=-g^{b} \cdot \nabla^{2}: C^{\infty}(L) \rightarrow C^{\infty}(L)
$$

Furthermore $\nabla=d$ and $\nabla^{*}=d^{*}$ so this Laplacian also coincides with the Hodge Laplacian $d^{*} d$. On differential $k$-forms the Levi-Civita $\nabla$-Laplacian and the Hodge Laplacian coincide only up to curvature terms.

To conclude, let us recall a few elements of functional analysis. We now let $E$ denote a Banach space. Then $E^{*}$ denotes its dual space and $\langle\cdot, \cdot\rangle$ denotes the duality map $E^{*} \times E \rightarrow \mathbb{R}$.

Let $P: E \rightarrow F$ be a continuous linear map between Banach spaces. Recall that the norm of $P$ is defined as $\|P\|:=\sup _{|e|=1}|P(e)|=\sup _{e \neq 0}$ $(|P(e)| /|e|)$. This implies that, $\forall e \neq 0,|P(e)| \leq\|P\| \cdot|e|$. If $P$ is injective and surjective then it follows from the Open Mapping Theorem that its inverse $P^{-1}$ is also continuous. In this case $\inf _{|e|=1}|P(e)|>0$ and we can 
calculate the norm of $P^{-1}$ as follows:

$$
\begin{aligned}
\left\|P^{-1}\right\| & =\sup _{f \neq 0} \frac{\left|P^{-1}(f)\right|}{|f|}=\sup _{e \neq 0} \frac{|e|}{|P(e)|}=\sup _{|e|=1} \frac{1}{|P(e)|} \\
& =\frac{1}{\inf _{|e|=1}|P(e)|} .
\end{aligned}
$$

Recall that, given any subspace $Z \leq F$, the annihilator of $Z$ is defined as

$$
\operatorname{Ann}(Z):=\left\{\phi \in F^{*}:\langle\phi, z\rangle=0, \forall z \in Z\right\}
$$

Note that $\operatorname{Ann}(\bar{Z})=\operatorname{Ann}(Z)$. Let $P^{*}: F^{*} \rightarrow E^{*}$ be the dual map, defined by $\left\langle P^{*}(\phi), e\right\rangle:=\langle\phi, P(e)\rangle$. It is simple to check that $\operatorname{Ann}(\operatorname{Im}(P))=\operatorname{Ker}\left(P^{*}\right)$.

Recall that the cokernel of $P$ is defined to be the quotient space Coker $(P):=F / \operatorname{Im}(P)$. Assume the image $\operatorname{Im}(P)$ of $P$ is a closed subspace of $F$, so that $\operatorname{Coker}(P)$ has an induced Banach space structure. The projection $\pi: F \rightarrow \operatorname{Coker}(P)$ is surjective so its dual map $\pi^{*}:(\operatorname{Coker}(P))^{*} \rightarrow F^{*}$ is injective. The image of $\pi^{*}$ coincides with the space $\operatorname{Ann}(\operatorname{Im}(P))$ so $\pi^{*}$ defines an isomorphism between $(\operatorname{Coker}(P))^{*}$ and $\operatorname{Ann}(\operatorname{Im}(P))$. We conclude that there exists a natural isomorphism $(\operatorname{Coker}(P))^{*} \simeq \operatorname{Ker}\left(P^{*}\right)$.

Remark 2.3. It is clear that $\operatorname{Ker}\left(P^{*}\right)$ can be characterized as follows:

$$
\phi \in \operatorname{Ker}\left(P^{*}\right) \Leftrightarrow\langle\phi, P(e)\rangle=0, \quad \forall e \in E
$$

On the other hand, the Hahn-Banach theorem shows that $f \in \bar{Z}$ iff $\langle\phi, f\rangle=$ $0, \forall \phi \in \operatorname{Ann}(Z)$. Applying this to $Z:=\operatorname{Im}(P)$, we find the following characterization of $\overline{\operatorname{Im}(P)}$ :

$$
f \in \overline{\operatorname{Im}(P)} \Leftrightarrow\langle\phi, f\rangle=0, \quad \forall \phi \in \operatorname{Ker}\left(P^{*}\right) .
$$

We say that $P$ is Fredholm if its image $\operatorname{Im}(P)$ is closed in $F$ and both $\operatorname{Ker}(P)$ and $\operatorname{Coker}(P)$ are finite-dimensional. We then define the index of $P$ to be

$$
i(P):=\operatorname{dim}(\operatorname{Ker}(P))-\operatorname{dim}(\operatorname{Coker}(P))=\operatorname{dim}(\operatorname{Ker}(P))-\operatorname{dim}\left(\operatorname{Ker}\left(P^{*}\right)\right) .
$$

Important remarks: Throughout this paper we will often encounter chains of inequalities of the form

$$
\left|e_{0}\right| \leq C_{1}\left|e_{1}\right| \leq C_{2}\left|e_{2}\right| \leq \ldots
$$


The constants $C_{i}$ will often depend on factors that are irrelevant within the given context. In this case we will sometimes simplify such expressions by omitting the subscripts of the constants $C_{i}$, i.e., by using a single constant $C$.

We assume all manifolds are oriented. In Part 2 of the paper, we will work under the assumption $m \geq 3$.

\section{Part 1. Sobolev Embedding Theorems}

The goal of this part is to provide a self-contained overview of certain aspects of the theory of weighted Sobolev spaces on Riemannian manifolds. Aside from the special case of "conifolds", discussed in Section 6 and which is wellknown, the point of view we present here applies to manifolds in general and we would not know where to find it in the literature. In Sections 4 and 5, we find it useful to separate the "scaling factor" $\rho$ from the "weight" $w$ : distinguishing them in this way appears not to be a standard choice in the literature, but we find it useful so as to emphasize their different roles in the theory.

\section{Review of the theory of standard Sobolev spaces}

We now introduce and discuss Sobolev spaces on manifolds. A good reference, which at times we follow closely, is Hebey [4].

Let $(E, \nabla)$ be a metric pair over $(L, g)$. The standard Sobolev spaces are defined by

$$
\begin{gathered}
W_{k}^{p}(E):=\text { Banach space completion of the space } \\
\left\{\sigma \in C^{\infty}(E):\|\sigma\|_{W_{k}^{p}}<\infty\right\}
\end{gathered}
$$

where $p \in[1, \infty), \quad k \geq 0$ and we use the norm $\|\sigma\|_{W_{k}^{p}}:=\left(\Sigma_{j=0}^{k}\right.$ $\left.\int_{L}\left|\nabla^{j} \sigma\right|^{p} \operatorname{vol}_{g}\right)^{1 / p}$. We will sometimes use $L^{p}$ to denote the space $W_{0}^{p}$.

Remark 3.1. At times we will want to emphasize the metric $g$ rather than the specific Sobolev spaces. In these cases, we will use the notation $\|\cdot\|_{g}$.

It is important to find conditions ensuring that two metrics $g, \hat{g}$ on $L$ (corresponding to Levi-Civita connections $\nabla, \hat{\nabla}$ ), define equivalent Sobolev norms, i.e., such that there exists $C>0$ with $(1 / C)\|\cdot\|_{g} \leq\|\cdot\|_{\hat{g}} \leq C\|\cdot\|_{g}$. In this case the corresponding two completions, i.e., the two spaces $W_{k}^{p}$, coincide. 
Definition 3.2. We say that two Riemannian metrics $g, \hat{g}$ on a manifold $L$ are equivalent if they satisfy the following assumptions:

A1: There exists $C_{0}>0$ such that

$$
\left(1 / C_{0}\right) g \leq \hat{g} \leq C_{0} g .
$$

A2: For all $j \geq 1$ there exists $C_{j}>0$ such that

$$
\left|\nabla^{j} \hat{g}\right|_{g} \leq C_{j}
$$

Remark 3.3. It may be useful to emphasize that the conditions of Definition 3.2 are symmetric in $g$ and $\hat{g}$. Assumption A1 is obviously symmetric. Assumption A2 is also symmetric. For $j=1$, for example, this follows from the following calculation which uses the fact that the connections are metric:

$$
|\nabla \hat{g}|_{g}=|\nabla \hat{g}-\hat{\nabla} \hat{g}|_{g}=|A(\hat{g})|_{g} \simeq|A(g)|_{\hat{g}}=|\hat{\nabla} g|_{\hat{g}},
$$

where $\simeq$ replaces multiplicative constants. Note that in Equation (3.2) $A$ is the difference of the induced connections on $T^{*} L \otimes T^{*} L$. This tensor depends linearly on the tensor defined as the difference of the connections on $T L$. It is simple to see that these two tensors have equivalent norms so that Assumption A2 provides a pointwise bound on the norms of either one. From here we easily obtain bounds on the norms of the tensor defined as the difference of the induced connections on any tensor product of $T L$ and $T^{*} L$. Similar statements hold for bounds on the derivatives of $A$.

Assumptions 1 and 2 can be unified as follows. Assume that, for all $j \geq 0$, there exists $C_{j}>0$ such that

$$
\left|\nabla^{j}(\hat{g}-g)\right|_{g} \leq C_{j} .
$$

As long as $C_{0}$ is sufficiently small, for $j=0$ this condition implies Assumption 1 . Since $\nabla^{j} g=0$, it is clear that for $j>0$ it is equivalent to Assumption 2.

Lemma 3.4. Assume $g, \hat{g}$ are equivalent. Then the Sobolev norms defined by $g$ and $\hat{g}$ are equivalent.

Proof. Consider the Sobolev spaces of functions on $L$. Recall that $\nabla u=d u$. This implies that the $W_{1}^{p}$ norms depend only pointwise on the metrics. In this case Assumption A1 is sufficient to ensure equivalence. In general, however, 
the $W_{k}^{p}$ norms use the induced connections on tensor bundles. For example, assume $j=2$. Then

$$
\left|\nabla^{2} u\right|=|(A+\hat{\nabla})(A+\hat{\nabla}) u| \leq\left|A^{2} u\right|+|A \cdot \hat{\nabla} u|+|\hat{\nabla}(A u)|+\left|\hat{\nabla}^{2} u\right|,
$$

where $A:=\nabla-\hat{\nabla}$ is the difference of the appropriate connections. It is clearly sufficient to obtain pointwise bounds on $A$ and its derivative $\hat{\nabla} A$. As mentioned in Remark 3.3, these follow from Assumption A2. The same is true for Sobolev spaces of sections of tensor bundles over $L$.

Now consider the Sobolev spaces of sections of $E$. Since we are not changing the connection on $E$, Assumption A1 ensures equivalence of the $W_{1}^{p}$ norms. The equivalence of the $W_{k}^{p}$ norms is proved as above.

For $p>1$ we define $p^{\prime}$ via

$$
\frac{1}{p}+\frac{1}{p^{\prime}}=1 \text {, i.e., } p^{\prime}=\frac{p}{p-1} .
$$

For $p \geq 1$ we define $p^{*}$ via

$$
\frac{1}{p^{*}}=\frac{1}{p}-\frac{1}{m}, \text { i.e., } p^{*}=\frac{m p}{m-p} .
$$

It is simple to check that

$$
\frac{1}{p^{*}}+\frac{1}{p^{\prime}}=\frac{m-1}{m}
$$

More generally, for $p \geq 1$ and $l=\{1,2, \ldots\}$ we define $p_{l}^{*}$ via

$$
\frac{1}{p_{l}^{*}}=\frac{1}{p}-\frac{l}{m}, \text { i.e., } p_{l}^{*}=\frac{m p}{m-l p},
$$

so that $p^{*}=p_{1}^{*}$. Note that $p_{l}^{*}$ is obtained by $l$ iterations of the operation

$$
p \mapsto p^{*}
$$

and that $\frac{1}{p_{l}^{*}}<\frac{1}{p_{l-1}^{*}}<\frac{1}{p}$, so if $p_{l}^{*}>0$ (equivalently, $l p<m$ ) then $p_{l}^{*}>p_{l-1}^{*}>$ $p$. In other words, under appropriate conditions $p_{l}^{*}$ increases with $l$.

The Sobolev Embedding Theorems come in two basic forms, depending on the product $l p$. The Sobolev Embedding Theorems, Part I concern the 
existence of continuous embeddings of the form

$$
W_{k+l}^{p}(E) \hookrightarrow W_{k}^{p_{l}^{*}}(E) \quad(\text { for } l p<m),
$$

i.e., the existence of some constant $C>0$ such that, $\forall \sigma \in W_{k+l}^{p}(E)$,

$$
\|\sigma\|_{W_{k}^{p_{l}^{*}}(E)} \leq C\|\sigma\|_{W_{k+l}^{p}(E)} .
$$

A standard argument based on Hölder's inequality then shows that $W_{k+l}^{p}$ $(E) \hookrightarrow W_{k}^{q}(E)$, for all $q \in\left[p, p_{l}^{*}\right]$. We call $C$ the Sobolev constant. In words, bounds on the higher derivatives of $\sigma$ enhance the integrability of $\sigma$. Otherwise said, one can sacrifice derivatives to improve integrability; the more derivatives one sacrifices, the larger the integrability range $\left[p, p_{l}^{*}\right]$.

The exceptional case of Part I concerns the existence of continuous embeddings of the form

$$
W_{k+l}^{p}(E) \hookrightarrow W_{k}^{q}(E) \quad(\text { for } l p=m), \quad \forall q \in[p, \infty) .
$$

The Sobolev Embedding Theorems, Part II concern the existence of continuous embeddings of the form

$$
W_{k+l}^{p}(E) \hookrightarrow C^{k}(E) \quad(\text { for } l p>m) .
$$

Roughly speaking, this means that one can sacrifice derivatives to improve regularity.

The validity of these theorems for a given manifold $(L, g)$ depends on its Riemannian properties. It is a useful fact that the properties of $(E, \nabla)$ play no extra role: more precisely, if an Embedding Theorem holds for functions on $L$, it then holds for sections of any metric pair $(E, \nabla)$. This is a consequence of the following result.

Lemma 3.5 Kato's inequality. Let $(E, \nabla)$ be a metric pair. Let $\sigma$ be a smooth section of $E$. Then, away from the zero set of $\sigma$,

$$
|d| \sigma|| \leq|\nabla \sigma|
$$

Proof.

$$
2|\sigma||d| \sigma||=\left.|d| \sigma\right|^{2}|=2(\nabla \sigma, \sigma) \leq 2| \nabla \sigma|| \sigma \mid
$$

The next result shows that if Part I holds in the simplest cases, it then holds in all cases. Likewise, the general case of Part II follows from combining the simplest cases of Part II with the general case of Part I. 


\section{Proposition 3.6.}

(1) Assume Part I, Equation (3.7), holds for all $p<m$ with $l=1$ and $k=0$. Then Part I holds for all $p$ and $l$ satisfying $l p<m$ and for all $k \geq 0$.

(2) Assume Part I, Equation (3.7), holds in all cases and that the exceptional case, Equation (3.9), holds for $l=1$ and $k=0$. Then the exceptional case holds for all $p$ and $l$ satisfying $l p=m$ and for all $k \geq 0$.

(3) Assume Part I, Equation (3.7), and the exceptional case, Equation (3.9), hold in all cases and that Part II, Equation (3.10), holds for all $p>m$ with $l=1$ and $k=0$. Then Part II holds for all $p$ and $l$ satisfying $l p>m$ and for all $k \geq 0$.

Proof. As discussed above, it is sufficient to prove that the result holds for functions: as a result of Kato's inequality it will then hold for arbitrary metric pairs $(E, \nabla)$.

(1) Assume $l=1$. Given $u \in W_{k+1}^{p}$, Kato's inequality shows that $|u| \ldots$ $\left|\nabla^{k} u\right| \in W_{1}^{p}$. Applying Part I to each of these then shows that $W_{k+1}^{p} \hookrightarrow W_{k}^{p^{*}}$. The general case follows from the composition of the embeddings

$$
W_{k+l}^{p} \hookrightarrow W_{k+l-1}^{p^{*}} \hookrightarrow W_{k+l-2}^{p_{2}^{*}} \hookrightarrow \ldots
$$

(2) For $l=1$ we can prove $W_{k+1}^{p} \hookrightarrow W_{k}^{q}$ as in (1) above. Now assume $l p=m$ for $l \geq 2$. Then Part I yields $W_{l}^{p} \hookrightarrow W_{1}^{p_{l-1}^{*}}$. Since $p_{l-1}^{*}=m$ we can now apply the exceptional case in its simplest form.

(3) Let us consider, for example, the case $l=2$ and $k=0$. We are then assuming that $p>m / 2$. Let us distinguish three subcases, as follows. Assume $p \in(m / 2, m)$. Then Part 1 implies that $W_{2}^{p} \hookrightarrow W_{1}^{p^{*}}$. Since $p^{*}>m$ we can now use the embedding $W_{1}^{p^{*}} \hookrightarrow C^{0}$ to conclude. Now assume $p=m$. Then $W_{2}^{p} \hookrightarrow W_{1}^{q}$ for any $q>m$ and we can conclude as above. Finally, assume $p>m$. Then $W_{2}^{p} \hookrightarrow W_{1}^{p} \hookrightarrow C^{0}$. The other cases are similar.

Corollary 3.7. Assume the Sobolev Embedding Theorems hold for $(L, g)$. Let $\hat{g}$ be a second Riemannian metric on $L$ such that, for some $C_{0}>0$, $\left(1 / C_{0}\right) g \leq \hat{g} \leq C_{0} g$. Then the Sobolev Embedding Theorems hold also for $(L, \hat{g})$. 
Proof. According to Proposition 3.6 it is sufficient to verify the Sobolev Embedding Theorems in the case $l=1$ and $k=0$. These involve only $C^{0}$ information on the metric. The conclusion is thus straightforward.

Remark 3.8. Under a certain density condition, Proposition 3.6 can be enhanced as follows.

Assume Part I, Equation (3.7), holds for $p=1, l=1$ and $k=0$, i.e., $W_{1}^{1} \hookrightarrow L^{\frac{m}{m-1}}$. Assume also that, for all $p<m$, the space $C_{c}^{\infty}(L)$ is dense in $W_{1}^{p}$. Then Part I holds for all $p<m$ with $l=1$ and $k=0$, i.e., $W_{1}^{p} \hookrightarrow L^{p^{*}}$. The proof is as follows.

Choose $u \in C_{c}^{\infty}(L)$. One can check that, for all $s>1,|u|^{s} \in W_{1}^{p}$, cf., e.g., [4]. Then, using Part I and Hölder's inequality,

$$
\begin{aligned}
\left\||u|^{s}\right\|_{L^{\frac{m}{m-1}}} & \leq C \int_{L}\left(|u|^{s}+\left.|\nabla| u\right|^{s} \mid\right) \operatorname{vol}_{g} \\
& \leq C \int_{L}\left(|u|^{s-1}|u|+|u|^{s-1}|\nabla u|\right) \operatorname{vol}_{g} \\
& \leq C\left\|\left.u\right|^{s-1}\right\|_{L^{p^{\prime}}}\left(\|u\|_{L^{p}}+\|\nabla u\|_{L^{p}}\right) .
\end{aligned}
$$

Let us now choose $s$ so that $(s-1) p^{\prime}=s m /(m-1)$, i.e., $s=p^{*}(m-1) / m$. Substituting, we find

$$
\left(\int_{L}|u|^{p^{*}}\right)^{\frac{m-1}{m}} \leq C\left(\int_{L}|u|^{p^{*}}\right)^{\frac{1}{p^{\prime}}}\|u\|_{W_{1}^{p}} .
$$

This leads to $\|u\|_{L^{p^{*}}} \leq C\|u\|_{W_{1}^{p}}$, for all $u \in C_{c}^{\infty}(L)$. By density, the same is true for all $u \in W_{1}^{p}$.

To conclude, we mention that if $(L, g)$ is complete, then $C_{c}^{\infty}(L)$ is known to be dense in $W_{1}^{p}$ for all $p \geq 1$, cf. [4] Theorem 3.1.

The most basic setting in which all parts of the Sobolev Embedding Theorems hold is when $L$ is a smooth bounded domain in $\mathbb{R}^{m}$ endowed with the standard metric $\tilde{g}$. Another important class of examples is the following.

Theorem 3.9. Assume $(L, g)$ satisfies the following assumptions: there exists $R_{1}>0$ and $R_{2} \in \mathbb{R}$ such that

$$
i(g) \geq R_{1}, \quad \operatorname{Ric}(g) \geq R_{2} g .
$$


Then:

(1) The Sobolev embeddings Part I, Equation (3.7), hold for all $p$ and $l$ satisfying $l p<m$ and for all $k \geq 0$.

(2) The exceptional case of Part I, Equation (3.9), holds for all $p$ and $l$ satisfying $l p=m$ and for all $k \geq 0$.

(3) The Sobolev embeddings Part II, Equation (3.10), hold for all $p$ and $l$ satisfying $l p>m$ and for all $k \geq 0$.

Furthermore, when $k p>m, W_{k}^{p}$ is a Banach algebra. Specifically, there exists $C>0$ such that, for all $u, v \in W_{k}^{p}$, the product uv belongs to $W_{k}^{p}$ and satisfies

$$
\|u v\|_{W_{k}^{p}} \leq C\|u\|_{W_{k}^{p}} \cdot\|v\|_{W_{k}^{p}}
$$

We will prove Theorem 3.9 below. Roughly speaking, the reason it holds is the following. Given any coordinate system on $L$, the embeddings hold on every chart endowed with the flat metric $\tilde{g}$. Now recall that, given any $(L, g)$ and any $x \in L$, it is always possible to find coordinates $\phi_{x}: B \subset \mathbb{R}^{m} \rightarrow L$ in which the metric $g$ is a small perturbation of the flat metric: this implies that the embeddings hold locally also with respect to $g$. The problem is that, in general, the size of the ball $B$, thus the corresponding Sobolev constants, will depend on $x$. Our assumptions on $L$, however, can be used to build a special coordinate system whose charts admit uniform bounds. One can then show that this implies that the embeddings hold globally. The main technical step in the proof of Theorem 3.9 is thus the following result concerning the existence and properties of harmonic coordinate systems.

Theorem 3.10. Assume $(L, g)$ satisfies the assumptions of Theorem 3.9. Then for all small $\epsilon>0$ there exists $r>0$ such that, for each $x \in L$, there exist coordinates $\phi_{x}: B_{r} \subset \mathbb{R}^{m} \rightarrow L$ satisfying

(1) $\phi_{x}^{-1}$ (seen as a map into $\mathbb{R}^{m}$ ) is harmonic.

(2) $\left\|\phi_{x}^{*} g-\tilde{g}\right\|_{C^{0}} \leq \epsilon$.

Remark 3.11. Theorem 3.10 can be heavily improved, cf. [4] Theorem 1.2. First, it is actually a local result, i.e., one can get similar results for any open subset of $L$ by imposing similar assumptions on a slightly larger subset. Secondly, these same assumptions actually yield certain $C^{0, \alpha}$ bounds. Thirdly, assumptions on the higher derivatives of the Ricci tensor yield certain bounds on the higher derivatives of $\phi_{x}^{*} g-\tilde{g}$, see Remark 4.6 for details. 
To conclude, it may be useful to emphasize that imposing a global lower bound on the injectivity radius of $(L, g)$ implies completeness.

Proof of Theorem 3.9. As seen in Proposition 3.6, it is sufficient to prove the Sobolev Embedding Theorems in the simplest cases. Concerning Part I, let us choose $u \in W_{1}^{p}(L)$. Using the coordinates of Theorem 3.10, $\phi_{x}^{*} u \in$ $W_{1}^{p}\left(B_{r}\right)$. All Sobolev Embedding Theorems hold on $B_{r}$ with its standard metric $\tilde{g}$. Thus there exists a constant $C$ such that, with respect to $\tilde{g}$,

$$
\left\|\phi_{x}^{*} u\right\|_{L^{p^{*}\left(B_{r}\right)}} \leq C\left\|\phi_{x}^{*} u\right\|_{W_{1}^{p}\left(B_{r}\right)}
$$

The fact that $\nabla u=d u$ implies that Equation (3.12) involves only $C^{0}$ information on the metric. Since $\phi_{x}^{*} g$ is $C^{0}$ close to $\tilde{g}$, up to a small change of the constant $C$ the same inequality holds with respect to $\phi_{x}^{*} g$. Let $B_{x}(r)$ denote the ball in $(L, g)$ with centre $x$ and radius $r$. Then $B_{x}(r / 2) \subset \phi_{x}\left(B_{r}\right) \subset$ $B_{x}(2 r)$ so

$$
\begin{aligned}
\int_{B_{x}(r / 2)}|u|^{p^{*}} \operatorname{vol}_{g} \leq & \int_{\phi_{x}\left(B_{r}\right)}|u|^{p^{*}} \operatorname{vol}_{g} \\
\leq & C\left(\int_{\phi_{x}\left(B_{r}\right)}\left(|u|^{p}+|d u|^{p}\right) \operatorname{vol}_{g}\right)^{\frac{p^{*}-p+p}{p}} \\
\leq & C\left(\int_{L}\left(|u|^{p}+|d u|^{p}\right) \operatorname{vol}_{g}\right)^{\frac{p^{*}-p}{p}} \\
& \times\left(\int_{B_{x}(2 r)}\left(|u|^{p}+|d u|^{p}\right) \operatorname{vol}_{g}\right)
\end{aligned}
$$

Let us now integrate both sides of the above equation with respect to $x \in L$. We can then change the order of integration according to the formula

$$
\int_{x \in L}\left(\int_{y \in B_{x}(r)} f(y) \operatorname{vol}_{g}\right) \operatorname{vol}_{g}=\int_{y \in L} f(y)\left(\int_{x \in B_{y}(r)} \operatorname{vol}_{g}\right) \operatorname{vol}_{g}
$$

Reducing $r$ if necessary, the $C^{0}$ estimate on $g$ yields uniform bounds (with respect to $x$ ) on $\operatorname{vol}_{g}\left(B_{x}(r / 2)\right)$ and $\operatorname{vol}_{g}\left(B_{x}(2 r)\right)$ because analogous bounds hold for $\tilde{g}$. This allows us to substitute the inner integrals with appropriate 
constants. We conclude that

$$
\begin{aligned}
\int_{L}|u|^{p^{*}} \operatorname{vol}_{g} & \leq C\left(\int_{L}\left(|u|^{p}+|d u|^{p}\right) \operatorname{vol}_{g}\right)^{\frac{p^{*}-p}{p}}\left(\int_{L}\left(|u|^{p}+|d u|^{p}\right) \operatorname{vol}_{g}\right) \\
& =C\left(\int_{L}\left(|u|^{p}+|d u|^{p}\right) \operatorname{vol}_{g}\right)^{\frac{p^{*}}{p}} .
\end{aligned}
$$

We conclude by raising both sides of the above equation to the power $1 / p^{*}$. Note that the final constant $C$ can be estimated in terms of the volume of balls in $L$ and of the constant $C$ appearing in Equation (3.12).

The exceptional case of Part I is similar: it is sufficient to replace $p^{*}$ with any $q>m$. Part II is also similar, although slightly simpler. Specifically, one finds as above that

$$
\|u\|_{C^{0}\left(\phi_{x}\left(B_{r}\right)\right)} \leq C\|u\|_{W_{1}^{p}\left(\phi_{x}\left(B_{r}\right)\right)} \leq C\|u\|_{W_{1}^{p}(L)} .
$$

Since this holds for all $x \in L$, we conclude that $\|u\|_{C^{0}(L)} \leq C\|u\|_{W_{1}^{p}(L)}$.

The proof that $W_{k}^{p}$ is a Banach algebra relies on the Sobolev Embedding Theorems and some simple algebraic manipulations. For brevity, we present only the case $W_{2}^{p}$ with $2 p>m$, which already contains all the main ideas; [1], Theorem 5.23, gives the general proof for domains in $\mathbb{R}^{m}$.

Recall the Leibniz rule

$$
\nabla^{j}(u v)=\sum_{k=0}^{j}\left(\begin{array}{l}
j \\
k
\end{array}\right)\left(\nabla^{k} u\right) \otimes\left(\nabla^{j-k} v\right)
$$

It thus suffices to estimate each term on the right-hand side, for $j=0,1,2$. The embedding $W_{2}^{p} \hookrightarrow C^{0}$ implies that

$$
\int_{L}|u v|^{p} \operatorname{vol}_{g} \leq\|u\|_{C^{0}}^{p} \cdot \int_{L}|v|^{p} \operatorname{vol}_{g} \leq C\|u\|_{W_{2}^{p}}^{p} \cdot\|v\|_{W_{2}^{p}}^{p}
$$

We can analogously estimate all other terms except perhaps $\int|\nabla u|^{p}|\nabla v|^{p}$. If $p>m$ we can use the stronger embedding $W_{2}^{p} \hookrightarrow C^{1}$ to estimate this term as above. Otherwise we use the following fact.

Fact: Assume $m / 2<p \leq m$. Then there exist $r, r^{\prime}$ such that $1 / r+1 / r^{\prime}=$ 1 and $p r<p^{*}, p r^{\prime}<p^{*}$.

This fact is obvious if $p=m$ (using the convention $p^{*}=\infty$ ). For $p<m$ it suffices to choose $r$ such that $m / p<r<m /(p-m)$ and $r^{\prime}=r /(r-1)$. 
The Sobolev Embedding Theorem, Part I, then yields $W_{1}^{p} \hookrightarrow L^{p r}$ so $|\nabla u|^{p} \in L^{r}$. Likewise, $|\nabla v|^{p} \in L^{r^{\prime}}$ so, using Hölder's inequality,

$$
\begin{aligned}
\int_{L}|\nabla u|^{p}|\nabla v|^{p} \operatorname{vol}_{g} & \leq\left\||\nabla u|^{p}\right\|_{L^{r}} \cdot\left\||\nabla v|^{p}\right\|_{L^{r^{\prime}}}=\|\nabla u\|_{L^{p r}}^{p} \cdot\|\nabla v\|_{L^{p r^{\prime}}}^{p} \\
& \leq C\|\nabla u\|_{W_{1}^{p}}^{p} \cdot\|\nabla v\|_{W_{1}^{p}}^{p} \leq C\|u\|_{W_{2}^{p}}^{p} \cdot\|v\|_{W_{2}^{p}}^{p} .
\end{aligned}
$$

Combining all these estimates proves that $\|u v\|_{W_{2}^{p}} \leq C\|u\|_{W_{2}^{p}} \cdot\|v\|_{W_{2}^{p}}$, as claimed.

Example 3.12. Any compact oriented Riemannian manifold $(L, g)$ satisfies the assumptions of Theorem 3.9. Thus the Sobolev Embedding Theorems hold in full generality for such manifolds. The same is true for the noncompact manifold $\mathbb{R}^{m}$, endowed with the standard metric $\tilde{g}$.

Let $\left(\Sigma, g^{\prime}\right)$ be a compact oriented Riemannian manifold. Consider $L:=$ $\Sigma \times \mathbb{R}$ endowed with the metric $\tilde{h}:=d z^{2}+g^{\prime}$. It is clear that $(L, \tilde{h})$ satisfies the assumptions of Theorem 3.9 so again the Sobolev Embedding Theorems hold in full generality for these manifolds. More generally they hold for the asymptotically cylindrical (A. Cyl.) manifolds of Section 6. Note however that here we are using the Sobolev spaces defined in Equation (3.1). In Section 6, we will verify the Sobolev Embedding Theorems for a different class of Sobolev spaces, cf. Definition 6.14.

\section{Scaled Sobolev spaces}

In applications standard Sobolev spaces are often not satisfactory for various reasons. First, they do not have good properties with respect to rescalings of the sort $\left(L, t^{2} g\right)$. Secondly, uniform geometric bounds of the sort seen in Theorem 3.9 are too strong. Thirdly, the finiteness condition in Equation (3.1) is very rigid and restrictive.

For all the above reasons it is often useful to modify the Sobolev norms. A simple way of addressing the first two problems is to introduce an extra piece of data, as follows.

Let $(L, g, \rho)$ be an oriented Riemannian manifold endowed with a scale factor $\rho>0$ or a scale function $\rho=\rho(x)>0$. Given any metric pair $(E, \nabla)$, the scaled Sobolev spaces are defined by

$$
\begin{aligned}
W_{k ; s c}^{p}(E):= & \text { Banach space completion of the space } \\
& \left\{\sigma \in C^{\infty}(E):\|\sigma\|_{W_{k ; s c}^{p}}<\infty\right\},
\end{aligned}
$$

where we use the norm $\|\sigma\|_{W_{k ; s c}^{p}}:=\left(\Sigma_{j=0}^{k} \int_{L}\left|\rho^{j} \nabla^{j} \sigma\right|_{g}^{p} \rho^{-m} \operatorname{vol}_{g}\right)^{1 / p}$. 
Note that at the scale $\rho \equiv 1$ these norms coincide with the standard norms.

Remark 4.1. Let us slightly change notation, using $g_{L}$ (respectively, $g_{E}$ ) to denote the metric on $L$ (respectively, on $E$ ). The metric $g$ used in the above norms to measure $\nabla^{j} \sigma$ is obtained by tensoring $g_{L}$ (applied to $\nabla^{j}$ ) with $g_{E}$ (applied to $\sigma$ ): let us write $g=g_{L} \otimes g_{E}$. We then find

$$
\left|\rho^{j} \nabla^{j} \sigma\right|_{g_{L} \otimes g_{E}} \rho^{-m} \operatorname{vol}_{g_{L} \otimes g_{E}}=\left|\nabla^{j} \sigma\right|_{\left(\rho^{-2} g_{L}\right) \otimes g_{E}} \operatorname{vol}_{\left(\rho^{-2} g_{L}\right) \otimes g_{E}}
$$

Roughly speaking, the scaled norms thus coincide with the standard norms obtained via the conformally equivalent metric $\rho^{-2} g_{L}$ on $L$. It is important to emphasize, however, that we are conformally rescaling only part of the metric. This can be confusing when $E$ is a tensor bundle over $L$, endowed with the induced metric: it would then be natural to also rescale the metric of $E$. We are also not changing the connections $\nabla$. In general these connections are not metric connections with respect to $\left(\rho^{-2} g_{L}\right) \otimes g_{E}$. This has important consequences regarding the Sobolev Embedding Theorems for scaled Sobolev spaces, as follows.

Naively, one might hope that such theorems hold under the assumptions:

$$
i\left(\rho^{-2} g\right) \geq R_{1}, \quad \operatorname{Ric}\left(\rho^{-2} g\right) \geq R_{2} \rho^{-2} g
$$

Indeed, these assumptions do suffice to prove the Sobolev Embedding Theorems in the simplest case, i.e., $l=1$ and $k=0$. However, the general case requires Kato's inequality, Lemma 3.5, which in turn requires metric connections. To prove these theorems we will thus need further assumptions on $\rho$, cf. Theorem 4.7 .

We now define rescaling to be an action of $\mathbb{R}^{+}$on the triple $(L, g, \rho)$, via $t \cdot(L, g, \rho):=\left(L, t^{2} g, t \rho\right)$. Recall that the Levi-Civita connection $\nabla$ on $L$ does not change under rescaling. Using this fact it is simple to check that $\|\sigma\|_{W_{k ; s c}^{p}}$, calculated with respect to $t \cdot(L, g, \rho)$, coincides with $\|\sigma\|_{W_{k ; s c}^{p}}$, calculated with respect to $(L, g, \rho)$ : in this sense the scaled norm is invariant under rescaling.

Remark 4.2. As in Remark 4.1, our definition of rescaling requires some care. To explain this, let us adopt the same notation as in Remark 4.1. Our notion of rescaling affects only the metric on $L$, not the metric on $E$. As 
before, this can be confusing when $E$ is a tensor bundle over $L$, endowed with the induced metric.

As in Section 3, it is important to find conditions under which $(L, g, \rho)$ and $(L, \hat{g}, \rho)$ define equivalent norms.

Definition 4.3. Let $(L, \rho)$ be a manifold endowed with a scale function. We say that two Riemannian metrics $g, \hat{g}$ are scaled-equivalent if they satisfy the following assumptions:

A1: There exists $C_{0}>0$ such that

$$
\left(1 / C_{0}\right) g \leq \hat{g} \leq C_{0} g
$$

A2: For all $j \geq 1$ there exists $C_{j}>0$ such that

$$
\left|\nabla^{j} \hat{g}\right|_{\rho^{-2} g \otimes g_{E}} \leq C_{j},
$$

where $\nabla$ is the Levi-Civita connection defined by $g, E=T^{*} L \otimes T^{*} L$ and we are using the notation introduced in Remark 4.1.

Remark 4.4. As in Remark 3.3, one can check that

$$
|\nabla \hat{g}|_{\rho^{-2} g \otimes g_{E}} \leq C_{1} \Rightarrow|A(\hat{g})|_{\rho^{-2} g \otimes g_{E}} \leq C_{1} .
$$

In turn this implies that $|A|_{\rho^{-2} g \otimes g_{E}} \leq C_{1}$, where now $A$ denotes the difference $\nabla-\hat{\nabla}$ of the connections on $T L$ and $E=T^{*} L \otimes T L$.

Again as in Remark 3.3, one can check that if for all $j \geq 0$ there exists $C_{j}>0$ such that

$$
\left|\nabla^{j}(\hat{g}-g)\right|_{\rho^{-2} g \otimes g_{E}} \leq C_{j}
$$

and if $C_{0}$ is sufficiently small then $g, \hat{g}$ satisfy Assumptions A1 and A2.

The following result is a simple consequence of Remark 4.1 and Lemma 3.4.

Lemma 4.5. Assume $(L, g, \rho),(L, \hat{g}, \rho)$ are scaled-equivalent in the sense of Definition 4.3. Then the scaled Sobolev norms are equivalent. 
We can also define the scaled spaces of $C^{k}$ sections

$$
C_{s c}^{k}(E):=\left\{\sigma \in C^{k}(E):\|\sigma\|_{C_{s c}^{k}}<\infty\right\}
$$

where we use the norm $\|\sigma\|_{C_{s c}^{k}}:=\sum_{j=0}^{k} \sup _{x \in L}\left|\rho^{j} \nabla^{j} \sigma\right|_{g}$. Once again, these norms define Banach spaces.

Remark 4.6. One can analogously define $C_{s c}^{k, \alpha}$ spaces. Note that Equation (4.2) implies that $C_{s c}^{0}=C^{0}$. It is these spaces which are relevant to the generalization to higher derivatives of Theorem 3.10. Specifically, bounds on the higher derivatives of $\operatorname{Ric}(g)$ yield $C_{s c}^{k, \alpha}$ bounds on $\phi_{x}^{*} g-\tilde{g}$ with respect to the (constant) scale factor $r$ determined by the theorem.

We are now ready to study the Sobolev Embedding Theorems for scaled spaces. As mentioned in Remark 4.1, these theorems require further assumptions on $\rho$.

Theorem 4.7. Let $(L, g)$ be a Riemannian manifold and $\rho$ a positive function on L. Assume there exist constants $R_{1}>0, R_{2} \in \mathbb{R}, R_{3}>1$ and $\zeta>0$ such that:

A1: $\forall x \in L, \quad i_{x}(g) \geq R_{1} \rho(x)$.

A2: $\forall x \in L, \quad \operatorname{Ric}_{x}(g) \geq R_{2} \rho(x)^{-2} g_{x}$.

A3: $\forall x \in L, \forall y \in B(x, \zeta \rho(x))$,

$$
\left(1 / R_{3}\right) \rho(x) \leq \rho(y) \leq R_{3} \rho(x)
$$

Then all parts of the Sobolev Embedding Theorems hold for scaled norms and for any metric pair $(E, \nabla)$. Furthermore, when $k p>m, W_{k ; s c}^{p}$ is a Banach algebra.

Now let $\hat{g}$ be a second Riemannian metric on $L$ such that, for some $C_{0}>0,\left(1 / C_{0}\right) g \leq \hat{g} \leq C_{0} g$. Then the scaled Sobolev Embedding Theorems hold also for $(L, \hat{g}, \rho)$ and for any metric pair $(E, \nabla)$. The Sobolev constants of $\hat{g}$ depend only on the Sobolev constants of $g$ and on $C_{0}$.

Proof. Let us prove Part 1 for functions, assuming $l=1, k=0$. Choose $x \in L$. Set $B_{x}:=B(x, \zeta \rho(x))$. For $y \in B_{x}$, consider the rescaled metric $h$ defined by $h_{y}:=\rho(x)^{-2} g_{y}$. Assumption A1 shows that $i_{y}(g) \geq R_{1} \rho(y)$. Using 
Assumption A3 we find

$$
i_{y}(h)=\rho(x)^{-1} i_{y}(g) \geq R_{1} \rho(y) \rho(x)^{-1} \geq R_{1} / R_{3} .
$$

Now recall that the Ricci curvature Ric is invariant under rescaling, i.e., $\operatorname{Ric}(h)=\operatorname{Ric}(g)$. Then Assumptions A2 and A3 show that

$$
\operatorname{Ric}_{y}(h)=\operatorname{Ric}_{y}(g) \geq R_{2} \rho(y)^{-2} \rho(x)^{2} h \geq\left(R_{2} / R_{3}^{2}\right) h .
$$

We have thus obtained lower bounds on the injectivity radius and Ricci curvature of $\left(B_{x}, h\right)$. Note that these bounds are independent of $x$. Recall from Remark 3.11 that Theorem 3.10 is essentially local. Specifically, set $B_{x}^{\prime}:=$ $B(x,(1 / 2) \zeta \rho(x))$. Then for any $\epsilon>0$ there exists $r=r\left(p, R_{1}, R_{2}, R_{3}, \epsilon, m\right)$ such that, for any $x \in L$, there exist coordinates $\phi_{x}: B_{r} \rightarrow\left(B_{x}^{\prime}, h\right)$ satisfying $\left\|\phi_{x}^{*} h-\tilde{g}\right\|_{C^{0}} \leq \epsilon$.

Exactly as in the proof of Theorem 3.9, we can now use the local Sobolev Embedding Theorems for $B_{r}$ to conclude that

$$
\left(\int_{B_{x}^{\prime}}|u|^{p^{*}} \operatorname{vol}_{h}\right)^{1 / p^{*}} \leq C\left(\int_{B_{x}^{\prime}}\left(|u|^{p}+|d u|_{h}^{p}\right) \operatorname{vol}_{h}\right)^{1 / p} .
$$

Assumption A3 allows us, up to a change of constants, to replace the (locally) constant quantity $\rho(x)$ with the function $\rho(y)$. Remark 4.1 shows how replacing $\rho^{-2} g$ with $g$ leads to the scaled norms. Proceeding as in the proof of Theorem 3.9, via double integration, we then get

$$
\|u\|_{L_{s c}^{p^{*}}} \leq C\|u\|_{W_{1 ; s c}^{p}}
$$

where we are now using the metric $g$.

Now consider the case $k=1$, i.e., assume $u \in W_{2 ; s c}^{p}$. Then $\phi_{x}^{*}|\nabla u|_{h} \in$ $W_{1}^{p}\left(B_{r}\right)$. As before, we obtain

$$
\left(\int_{B_{x}^{\prime}}|\nabla u|_{h}^{p^{*}} \operatorname{vol}_{h}\right)^{1 / p^{*}} \leq C\left(\int_{B_{x}^{\prime}}\left(|\nabla u|_{h}^{p}+\left|d\left(|\nabla u|_{h}\right)\right|_{h}^{p}\right) \operatorname{vol}_{h}\right)^{1 / p} .
$$


Note that the Levi-Civita connections of $g$ and $h$ coincide. We can thus apply Kato's inequality, finding $\left.\left.|d| \nabla u\right|_{h}\right|_{h} \leq\left|\nabla^{2} u\right|_{h}=\left|\rho(x)^{2} \nabla^{2} u\right|_{g}$. This leads to

$$
\begin{aligned}
& \left(\int_{B_{x}^{\prime}}|\rho(x) \nabla u|_{g}^{p^{*}} \rho(x)^{-m} \operatorname{vol}_{g}\right)^{1 / p^{*}} \\
& \quad \leq C\left(\int_{B_{x}^{\prime}}\left(|\rho(x) \nabla u|_{g}^{p}+\left|\rho(x)^{2} \nabla^{2} u\right|_{g}^{p}\right) \rho(x)^{-m} \operatorname{vol}_{g}\right)^{1 / p} .
\end{aligned}
$$

We can now proceed as before, using Assumption A3, to obtain

$$
\|\nabla u\|_{L_{s c}^{p^{*}}} \leq C\|\nabla u\|_{W_{1 ; s c}^{p}}
$$

Together with Equation (4.4), this implies $W_{2 ; s c}^{p} \hookrightarrow W_{1 ; s c}^{p^{*}}$.

The other cases and parts of the Sobolev Embedding Theorems can be proved analogously.

The claim that $W_{k ; s c}^{p}$ is a Banach algebra can be proved as in Theorem 3.9, using Remark 4.1 to write the scaled norms in terms of standard norms. In this case, the fact that the connection $\nabla$ is not a metric connection with respect to the rescaled metric $\rho^{-2} g$ is not a problem: the proof only uses the Leibniz rule (together with Hölder's inequality for $L^{p}$ norms and the Sobolev Embedding Theorems which we have just proved).

The proof of the Sobolev Embedding Theorems for $(L, \hat{g}, \rho)$ is similar. For example, to prove Part I with $l=1$ and $k=0$, we locally define $\hat{h}_{y}:=\rho^{-2}(x) \hat{g}_{y}$. Our assumption on $\hat{g}$ allows us to substitute $h$ with $\hat{h}$ in Equation (4.3). The proof then continues as before. Now consider the case $k=1$, i.e., assume $u \in W_{2 ; s c}^{p}$ with respect to $\hat{g}$. Let $\hat{\nabla}$ denote the Levi-Civita connection defined by $\hat{g}$. We can then study $\phi_{x}^{*}|\hat{\nabla} u|_{\hat{h}}$ as before, obtaining the analogue of Equation (4.5) in terms of $(\hat{h}, \hat{\nabla})$ instead of $(h, \nabla)$. Since the Levi-Civita connections of $\hat{g}$ and $\hat{h}$ coincide we also obtain the analogue of Equation (4.6). The proof then continues as before.

Remark 4.8. Compare the proof of Theorem 4.7 with the ideas of Remark 4.1. The main issue raised in Remark 4.1 concerned Kato's inequality for the rescaled metric $\rho^{-2} g$. In the proof of the theorem this problem is solved by Assumption A3, which essentially allows us to locally treat $\rho$ as a constant. Assumptions A1 and A2 are then similar to the assumptions of Remark 4.1.

Example 4.9. We now want to present two important examples of $(L, g, \rho)$ satisfying Assumptions A1-A3 of Theorem 4.7. 
(1) Let $L$ be a smooth bounded domain in $\mathbb{R}^{m}$, endowed with the standard metric $\tilde{g}$. Given any $x \in L$ we can define $\rho(x):=d(x, \partial L)$. This function satisfies Assumption A1 with $R_{1}=1$ and Assumption A2 with $R_{2}=0$. The triangle inequality shows that, for all $y \in B(x,(1 / 2) \rho(x))$, $(1 / 2) \rho(x) \leq \rho(y) \leq(3 / 2) \rho(x)$. This implies that Assumption A3 is also satisfied.

(2) Given a compact oriented Riemannian manifold $\left(\Sigma, g^{\prime}\right)$, let $L:=\Sigma \times$ $(0, \infty)$ and $\tilde{g}:=d r^{2}+r^{2} g^{\prime}$. Let $\theta$ denote the generic point on $\Sigma$. There is a natural action

$$
\mathbb{R}^{+} \times L \rightarrow L, \quad t \cdot(\theta, r):=(\theta, t r) .
$$

Given any $t \in \mathbb{R}^{+}$, it is simple to check that $t^{*} \tilde{g}=t^{2} \tilde{g}$. For any $x \in L$, note that $i_{t x}(\tilde{g})=i_{x}\left(t^{*} \tilde{g}\right)$. We conclude that $i_{t x}(\tilde{g})=t i_{x}(\tilde{g})$. Analogously, $\operatorname{Ric}_{t x}(\tilde{g})=\operatorname{Ric}_{x}(\tilde{g})$. It follows that, given any strictly positive $f=f(\theta)$, the function $\rho(\theta, r):=r f(\theta)$ satisfies A1 and A2. It is simple to check that it also satisfies Assumption A3. The simplest example is $f(\theta) \equiv 1$, i.e., $\rho(\theta, r)=r$. In Section 6 , we will extend this example to the category of "conifolds".

Remark 4.10. Since the norms $\|\cdot\|_{W_{k ; s c}^{p}}$ are scale-invariant it is clear that if the Sobolev Embedding Theorems hold for $(L, g, \rho)$ then they also hold for $\left(L, t^{2} g, t \rho\right)$ with the same Sobolev constants. This is reflected in the fact that Assumptions A1-A3 of Theorem 4.7 are scale-invariant.

\section{Weighted Sobolev spaces}

In Section 4, we mentioned that the finiteness condition determined by the standard Sobolev norms is very restrictive. This problem can be addressed by introducing a weight function $w=w(x)>0$ into the integrand. Coupling weights with scale functions then produces very general and useful spaces, as follows.

Let $(L, g)$ be a Riemannian manifold endowed with two positive functions $\rho$ and $w$. Given any metric pair $(E, \nabla)$, the weighted Sobolev spaces are defined by

$$
\begin{aligned}
W_{k ; w}^{p}(E):= & \text { Banach space completion of the space } \\
& \left\{\sigma \in C^{\infty}(E):\|\sigma\|_{W_{k ; w}^{p}}<\infty\right\}
\end{aligned}
$$

where we use the norm $\|\sigma\|_{W_{k ; w}^{p}}:=\left(\Sigma_{j=0}^{k} \int_{L}\left|w \rho^{j} \nabla^{j} \sigma\right|_{g}^{p} \rho^{-m} \operatorname{vol}_{g}\right)^{1 / p}$. 
We can also define the weighted spaces of $C^{k}$ sections

$$
C_{w}^{k}(E):=\left\{\sigma \in C^{k}(E):\|\sigma\|_{C_{w}^{k}}<\infty\right\}
$$

where we use the norm $\|\sigma\|_{C_{w}^{k}}:=\sum_{j=0}^{k} \sup _{x \in L}\left|w \rho^{j} \nabla^{j} \sigma\right|_{g}$. Once again, these norms define Banach spaces.

Theorem 5.1. Let $(L, g)$ be a Riemannian manifold endowed with positive functions $\rho$ and $w$. Assume $\rho$ satisfies the assumptions of Theorem 4.7 with respect to constants $R_{1}, R_{2}, R_{3}$ and $\zeta$. Assume also that there exists a positive constant $R_{4}$ such that, $\forall x \in L, \forall y \in B(x, \zeta \rho(x))$,

$$
\left(1 / R_{4}\right) w(x) \leq w(y) \leq R_{4} w(x)
$$

Then all parts of the Sobolev Embedding Theorems hold for the weighted norms defined by $(\rho, w)$ and for any metric pair $(E, \nabla)$.

Now let $\hat{g}$ be a second Riemannian metric on $L$ such that, for some $C_{0}>$ $0,\left(1 / C_{0}\right) g \leq \hat{g} \leq C_{0} g$. Then the weighted Sobolev Embedding Theorems hold also for $(L, \hat{g}, \rho, w)$ and for any metric pair $(E, \nabla)$. The Sobolev constants of $\hat{g}$ depend only on the Sobolev constants of $g$ and on $C_{0}$.

Proof. The proof is a small modification of the proof of Theorem 4.7: one needs simply to take into account the weights by multiplying Equations (4.3) and (4.6) by $w(x)$. The assumption on $w$ allows us, up to a change of constants, to replace the (locally) constant quantity $w(x)$ with the function $w(y)$.

Remark 5.2. Choose any constant $\beta \in \mathbb{R}$. Define rescaling to be an action of $\mathbb{R}^{+}$on $(L, g, \rho, w)$, via $t \cdot(L, g, \rho, w):=\left(L, t^{2} g, t \rho, t^{\beta} w\right)$. Then $\|\sigma\|_{W_{k ; w}^{p}}$, calculated with respect to $t \cdot(L, g, \rho, w)$, coincides with $t^{\beta}\|\sigma\|_{W_{k ; w}^{p}}$, calculated with respect to $(L, g, \rho, w)$ : this shows that these weighted norms are in general not invariant under rescaling. However, if the Sobolev Embedding Theorems hold for $(L, g, \rho, w)$ then, multiplying by the factor $t^{\beta}$, we see that they hold for $\left(L, t^{2} g, t \rho, t^{\beta} w\right)$ with the same Sobolev constant. This is reflected in the fact that the hypotheses of Theorem 5.1 are $t$-invariant.

\section{Application: manifolds with ends modelled on cones and cylinders}

We now introduce the category of "conifolds". These Riemannian manifolds are a well-known example for the theory of weighted Sobolev spaces. They 
will also provide a useful framework for our study of desingularizations. It will also be useful to define the analogous "cylindrical" category, both for its affinities to conifolds and as a tool for studying them.

Definition 6.1. Let $L^{m}$ be a smooth manifold. We say $L$ is a manifold with ends if it satisfies the following conditions:

1) We are given a compact subset $K \subset L$ such that $S:=L \backslash K$ has a finite number of connected components $S_{1}, \ldots, S_{e}$, i.e., $S=\amalg_{i=1}^{e} S_{i}$.

2) For each $S_{i}$ we are given a connected $(m-1)$-dimensional compact manifold $\Sigma_{i}$ without boundary.

3) There exist diffeomorphisms $\phi_{i}: \Sigma_{i} \times[1, \infty) \rightarrow \overline{S_{i}}$.

We then call the components $S_{i}$ the ends of $L$ and the manifolds $\Sigma_{i}$ the links of $L$. We denote by $\Sigma$ the union of the links of $L$.

Definition 6.2. Let $L$ be a manifold with ends. Let $g$ be a Riemannian metric on $L$. Choose an end $S_{i}$ with corresponding link $\Sigma_{i}$.

We say that $S_{i}$ is a conically singular (CS) end if the following conditions hold:

1) $\Sigma_{i}$ is endowed with a Riemannian metric $g_{i}^{\prime}$.

We then let $(\theta, r)$ denote the generic point on the product manifold $C_{i}:=\Sigma_{i} \times(0, \infty)$ and $\tilde{g}_{i}:=d r^{2}+r^{2} g_{i}^{\prime}$ denote the corresponding conical metric on $C_{i}$.

2) There exist a constant $\nu_{i}>0$ and a diffeomorphism $\phi_{i}: \Sigma_{i} \times(0, \epsilon] \rightarrow$ $\overline{S_{i}}$ such that, as $r \rightarrow 0$ and for all $k \geq 0$,

$$
\left|\widetilde{\nabla}^{k}\left(\phi_{i}^{*} g-\tilde{g}_{i}\right)\right|_{\tilde{g}_{i}}=O\left(r^{\nu_{i}-k}\right),
$$

where $\widetilde{\nabla}$ is the Levi-Civita connection on $C_{i}$ defined by $\tilde{g}_{i}$.

We say that $S_{i}$ is an $\mathrm{AC}$ end if the following conditions hold:

1) $\Sigma_{i}$ is endowed with a Riemannian metric $g_{i}^{\prime}$.

We again let $(\theta, r)$ denote the generic point on the product manifold $C_{i}:=\Sigma_{i} \times(0, \infty)$ and $\tilde{g}_{i}:=d r^{2}+r^{2} g_{i}^{\prime}$ denote the corresponding conical metric on $C_{i}$. 
2) There exist a constant $\nu_{i}<0$ and a diffeomorphism $\phi_{i}: \Sigma_{i} \times[R, \infty) \rightarrow$ $\overline{S_{i}}$ such that, as $r \rightarrow \infty$ and for all $k \geq 0$,

$$
\left|\widetilde{\nabla}^{k}\left(\phi_{i}^{*} g-\tilde{g}_{i}\right)\right|_{\tilde{g}_{i}}=O\left(r^{\nu_{i}-k}\right),
$$

where $\widetilde{\nabla}$ is the Levi-Civita connection on $C_{i}$ defined by $\tilde{g}_{i}$.

In either of the above situations we call $\nu_{i}$ the convergence rate of $S_{i}$.

Remark 6.3. Let $(L, g)$ be a manifold with ends. Assume $S_{i}$ is an AC end as in Definition 6.2. Using the notation of Remark 4.1 we can rewrite this condition as follows: for all $k \geq 0$,

$$
\left|\widetilde{\nabla}^{k}\left(\phi_{i}^{*} g-\tilde{g}_{i}\right)\right|_{r^{-2} \tilde{g}_{i} \otimes \tilde{g}_{i}}=O\left(r^{\nu_{i}}\right) .
$$

In particular there exist constants $C_{k}>0$ such that

$$
\left|\widetilde{\nabla}^{k}\left(\phi_{i}^{*} g-\tilde{g}_{i}\right)\right|_{r^{-2}} \tilde{g}_{i} \otimes \tilde{g}_{i} \leq C_{k} R^{\nu_{i}}
$$

By making $R$ larger if necessary, we can assume $C_{0} R^{\nu_{i}}$ is small. This implies that $\phi_{i}^{*} g$ and $\tilde{g}_{i}$ are scaled-equivalent in the sense of Definition 4.3, cf. Remark 4.4. The above conditions are stable under duality and tensor products so one can prove that, for any tensor $\sigma$ on $L$ and as $r \rightarrow \infty$,

$$
|\sigma|_{\phi_{i}^{*} g}=|\sigma|_{\tilde{g}_{i}}\left(1+O\left(r^{\nu_{i}}\right)\right) .
$$

If $\sigma=d f$ for some function $f$ on $L$, we can multiply both sides by $r$ to obtain an analogous estimate in terms of the rescaled metrics:

$$
|d f|_{r^{-2} \phi_{i}^{*} g}=|d f|_{r^{-2} \tilde{g}_{i}}\left(1+O\left(r^{\nu_{i}}\right)\right) .
$$

Furthermore, let $A:=\nabla-\widetilde{\nabla}$ denote the difference of the two connections defined by $\phi_{i}^{*} g$ and $\tilde{g}_{i}$. Then, as in Remark 3.3, Definition 6.2 implies that $|A|_{\tilde{g}_{i}}=O\left(r^{\nu_{i}-1}\right)$. This leads to

$$
\begin{aligned}
\left|\nabla^{2} f\right|_{\phi_{i}^{*} g} & =\left|\widetilde{\nabla}^{2} f\right|_{\tilde{g}_{i}}\left(1+O\left(r^{\nu_{i}}\right)\right)+|d f|_{\tilde{g}_{i}} O\left(r^{\nu_{i}-1}\right), \\
\left|\operatorname{tr}_{\phi_{i}^{*} g} \nabla^{2} f\right| & =\left|\operatorname{tr}_{\tilde{g}_{i}} \widetilde{\nabla}^{2} f\right|\left(1+O\left(r^{\nu_{i}}\right)\right)+|d f|_{\tilde{g}_{i}} O\left(r^{\nu_{i}-1}\right) .
\end{aligned}
$$

Multiplying these equations by $r^{2}$ we can re-write them as

$$
\begin{aligned}
\left|\nabla^{2} f\right|_{r^{-2} \phi_{i}^{*} g} & =\left|\widetilde{\nabla}^{2} f\right|_{r^{-2} \tilde{g}_{i}}+O\left(r^{\nu_{i}}\right)\left(\left|\widetilde{\nabla}^{2} f\right|_{r^{-2} \tilde{g}_{i}}+|d f|_{r^{-2} \tilde{g}_{i}}\right), \\
\left|r^{2} \Delta_{\phi_{i}^{*} g} f\right| & =\left|r^{2} \Delta_{\tilde{g}_{i}} f\right|+O\left(r^{\nu_{i}}\right)\left(\left|r^{2} \Delta_{\tilde{g}_{i}} f\right|+|d f|_{r^{-2} \tilde{g}_{i}}\right) .
\end{aligned}
$$

Analogous comments apply to higher derivatives and to CS ends. 
Definition 6.4. Let $(L, g)$ be a manifold with ends endowed with a Riemannian metric. We say that $L$ is a CS (respectively, AC) manifold if all ends are CS (respectively, AC). We say that $L$ is a CS/AC manifold if all ends are either $\mathrm{CS}$ or AC. We use the generic term conifold to indicate any CS, AC or CS/AC manifold.

When working with a CS/AC manifold we will often index the CS ("small") ends with numbers $\{1, \ldots, s\}$ and the AC ("large") ends with numbers $\{1, \ldots, l\}$. Furthermore we will denote the union of the CS links (respectively, of the CS ends) by $\Sigma_{0}$ (respectively, $S_{0}$ ) and those corresponding to the $\mathrm{AC}$ links and ends by $\Sigma_{\infty}, S_{\infty}$.

Remark 6.5. It is useful to include smooth compact manifolds in the category of conifolds: they are precisely those for which the set of ends is empty.

We now need to choose which function spaces to work with on conifolds. It turns out that the most useful classes of function spaces are precisely those of Section 5. One needs only to choose appropriate functions $\rho$ and $w$ satisfying the assumptions of Theorem 5.1, as follows.

Regarding notation, given a vector $\boldsymbol{\beta}=\left(\beta_{1}, \ldots, \beta_{e}\right) \in \mathbb{R}^{e}$ and $j \in \mathbb{N}$ we set $\boldsymbol{\beta}+j:=\left(\beta_{1}+j, \ldots, \beta_{e}+j\right)$. We write $\boldsymbol{\beta} \geq \hat{\boldsymbol{\beta}}$ iff $\beta_{i} \geq \hat{\beta}_{i}$ for all $i=1, \ldots, e$.

Definition 6.6. Let $L$ be a conifold with metric $g$. We say that a smooth function $\rho: L \rightarrow(0, \infty)$ is a radius function if $\phi_{i}^{*} \rho=r$, where $\phi_{i}$ are the diffeomorphisms of Definition 6.2. Given any vector $\boldsymbol{\beta}=\left(\beta_{1}, \ldots, \beta_{e}\right) \in \mathbb{R}^{e}$, choose a function $\boldsymbol{\beta}: L \rightarrow \mathbb{R}$ which, on each end $S_{i}$, restricts to the constant $\beta_{i}$. Then $\rho$ and $w:=\rho^{-\beta}$ satisfy the assumptions of Theorem 5.1, cf. Example 4.9. We call $(L, g, \rho, \boldsymbol{\beta})$ a weighted conifold.

Given any metric pair $(E, \nabla)$, we define weighted spaces $C_{\boldsymbol{\beta}}^{k}(E)$ and $W_{k, \boldsymbol{\beta}}^{p}(E)$ as in Section 5. We can equivalently define the space $C_{\boldsymbol{\beta}}^{k}(E)$ to be the space of sections $\sigma \in C^{k}(E)$ such that $\left|\nabla^{j} \sigma\right|=O\left(r^{\boldsymbol{\beta}-j}\right)$ as $r \rightarrow 0$ (respectively, $r \rightarrow \infty$ ) along each CS (respectively, AC) end.

In the case of a $\mathrm{CS} / \mathrm{AC}$ manifold we will often separate the $\mathrm{CS}$ and AC weights, writing $\boldsymbol{\beta}=(\boldsymbol{\mu}, \boldsymbol{\lambda})$ for some $\boldsymbol{\mu} \in \mathbb{R}^{s}$ and some $\boldsymbol{\lambda} \in \mathbb{R}^{l}$. We then write $C_{(\boldsymbol{\mu}, \boldsymbol{\lambda})}^{k}(E)$ and $W_{k,(\boldsymbol{\mu}, \boldsymbol{\lambda})}^{p}(E)$.

One can extend to these weighted spaces many results valid for standard Sobolev spaces. Hölder's inequality is one example. 
Lemma 6.7 (Weighted Hölder's inequality). Let $(L, g)$ be a conifold. Then, for all $p>1$ and $\boldsymbol{\beta}=\boldsymbol{\beta}_{\mathbf{1}}+\boldsymbol{\beta}_{\mathbf{2}}$,

$$
\|u v\|_{L_{\boldsymbol{\beta}}^{1}} \leq\|u\|_{L_{\boldsymbol{\beta}_{1}}^{p}} \cdot\|v\|_{L_{\boldsymbol{\beta}_{\mathbf{2}}}^{p^{\prime}}} .
$$

More generally, assume $\frac{1}{q}=\frac{1}{q_{1}}+\frac{1}{q_{2}}$. Then

$$
\|u v\|_{L_{\boldsymbol{\beta}}^{q}} \leq\|u\|_{L_{\boldsymbol{\beta}_{1}}^{q_{1}}} \cdot\|v\|_{L_{\boldsymbol{\beta}_{\mathbf{2}}}^{q_{2}}}
$$

Proof.

$$
\begin{aligned}
\|u v\|_{L_{\boldsymbol{\beta}}^{1}} & =\int_{L}\left(\rho^{-\boldsymbol{\beta}_{1}} u \rho^{-m / p}\right)\left(\rho^{-\boldsymbol{\beta}_{\mathbf{2}}} v \rho^{-m / p^{\prime}}\right) \operatorname{vol}_{g} \\
& \leq\left\|\rho^{-\boldsymbol{\beta}_{1}} u \rho^{-m / p}\right\|_{L^{p}} \cdot\left\|\rho^{-\boldsymbol{\beta}_{\mathbf{2}}} v \rho^{-m / p^{\prime}}\right\|_{L^{p^{\prime}}} \\
& =\|u\|_{L_{\boldsymbol{\beta}_{\mathbf{1}}}^{p}} \cdot\|v\|_{L_{\boldsymbol{\beta}_{\mathbf{2}}}^{p^{\prime}}}
\end{aligned}
$$

The general case is similar.

Corollary 6.8. Let $(L, g, \boldsymbol{\beta})$ be a weighted conifold. Then all parts of the weighted Sobolev Embedding Theorems hold for any metric pair $(E, \nabla)$.

Furthermore, assume $k p>m$. Then the corresponding weighted Sobolev spaces are closed under multiplication, in the following sense. For any $\boldsymbol{\beta}_{1}$ and $\boldsymbol{\beta}_{\mathbf{2}}$ there exists $C>0$ such that, for all $u \in W_{k, \boldsymbol{\beta}_{\mathbf{1}}}^{p}$ and $v \in W_{k, \boldsymbol{\beta}_{\mathbf{2}}}^{p}$,

$$
\|u v\|_{W_{k, \boldsymbol{\beta}_{\mathbf{1}}+\boldsymbol{\beta}_{\mathbf{2}}}^{p}} \leq C\|u\|_{W_{k, \boldsymbol{\beta}_{1}}^{p}} \cdot\|v\|_{W_{k, \boldsymbol{\beta}_{\mathbf{2}}}^{p}} .
$$

Proof. Let $(L, g)$ be a conifold. Write $L=K \cup S$ as in Definition 6.1 and let $C_{i}$ denote the cone corresponding to the end $S_{i}$. Example 4.9 showed that the assumptions for the scaled Sobolev Embedding Theorems hold for $\left(C_{i}, \tilde{g}_{i}, r\right)$. The same is true for the weighted Sobolev Embedding Theorems. Using the compactness of $K$ we conclude that these assumptions, thus the theorems, hold for $L$ with respect to any metric $\hat{g}$ such that $\phi_{i}^{*} \hat{g}=\tilde{g}_{i}$ on each end. As in Remark 6.3 one can assume that $\phi_{i}^{*} g$ and $\tilde{g}_{i}$ are scaledequivalent so there exists $C_{0}>0$ such that $\left(1 / C_{0}\right) \tilde{g}_{i} \leq \phi_{i}^{*} g \leq C_{0} \tilde{g}_{i}$. Again using the compactness of $K$ we may thus assume that $\left(1 / C_{0}\right) \hat{g} \leq g \leq C_{0} \hat{g}$. Theorem 5.1 now shows that the weighted Sobolev Embedding Theorems hold for $(L, g)$. The fact that weighted Sobolev spaces are closed with respect to products can be proved as in Theorem 4.7, using Lemma 6.7.

Remark 6.9. Let $(L, g)$ be an AC manifold. Note that for $\hat{\boldsymbol{\beta}} \geq \boldsymbol{\beta}$ there exist continuous embeddings $W_{k, \boldsymbol{\beta}}^{r} \hookrightarrow W_{k, \hat{\boldsymbol{\beta}}}^{r}$. The analogous statement is true for 
the weighted $C^{k}$ spaces. By composition Corollary 6.8 thus leads to the following statements:

1) If $l p<m$ then there exists a continuous embedding $W_{k+l, \boldsymbol{\beta}}^{p}(E) \hookrightarrow$ $W_{k, \hat{\boldsymbol{\beta}}}^{p_{l}^{*}}(E)$.

2) If $l p=m$ then, for all $q \in[p, \infty)$, there exist continuous embeddings $W_{k+l, \boldsymbol{\beta}}^{p}(E) \hookrightarrow W_{k, \hat{\boldsymbol{\beta}}}^{q}(E)$.

3) If $l p>m$ then there exists a continuous embedding $W_{k+l, \boldsymbol{\beta}}^{p}(E) \hookrightarrow$ $C_{\hat{\boldsymbol{\beta}}}^{k}(E)$.

Note that if $(L, g)$ is a CS manifold then the behaviour on the ends is studied in terms of $r \rightarrow 0$ rather than $r \rightarrow \infty$. In this case the same conclusions hold for the opposite situation $\hat{\boldsymbol{\beta}} \leq \boldsymbol{\beta}$. Finally, let $(L, g)$ be a CS/AC manifold with $\boldsymbol{\beta}=(\boldsymbol{\mu}, \boldsymbol{\lambda})$. Then the same conclusions hold for all $\hat{\boldsymbol{\beta}}=(\hat{\boldsymbol{\mu}}, \hat{\boldsymbol{\lambda}})$ with $\hat{\mu} \leq \boldsymbol{\mu}, \hat{\lambda} \geq \lambda$.

We now want to show that all the above notions and results are scaleindependent, as long as we rescale the weight function correctly to take into account the possibility of variable weights. We start by examining the properties of $\left(L, t^{2} g\right)$.

Lemma 6.10. Let $(L, g)$ be a conifold. For each $A C$ end $S_{i}$ let $\phi_{i}: \Sigma_{i} \times$ $[R, \infty) \rightarrow \overline{S_{i}}$ denote the diffeomorphism of Definition 6.2. In particular, for all $k \geq 0$ there exist $C_{k}>0$ such that, for $r \geq R$,

$$
\left|\widetilde{\nabla}^{k}\left(\phi_{i}^{*} g-\tilde{g}_{i}\right)\right|_{r^{-2}} \tilde{g}_{i} \otimes \tilde{g}_{i} \leq C_{k} r^{\nu_{i}} \leq C_{k} R^{\nu_{i}} .
$$

As seen in Remark 6.3, we can thus assume that $\phi_{i}^{*} g, \tilde{g}_{i}$ are scaled-equivalent.

Choose any $t>0$. Define the diffeomorphism

$$
\phi_{t, i}: \Sigma_{i} \times[t R, \infty) \rightarrow \overline{S_{i}}, \quad \phi_{t, i}(\theta, r):=\phi_{i}(\theta, r / t) .
$$

Then, for $r \geq t R$ and with respect to the same $C_{k}$, there are $t$-uniform estimates

$$
\left|\widetilde{\nabla}^{k}\left(\phi_{t, i}^{*}\left(t^{2} g\right)-\tilde{g}_{i}\right)\right|_{r^{-2} \tilde{g}_{i} \otimes \tilde{g}_{i}} \leq C_{k}(r / t)^{\nu_{i}} \leq C_{k} R^{\nu_{i}} .
$$

Analogously, for each CS end $S_{i}$ let $\phi_{i}$ denote the diffeomorphism of Definition 6.2. Define the diffeomorphism

$$
\phi_{t, i}: \Sigma_{i} \times(0, t \epsilon] \rightarrow \overline{S_{i}}, \quad \phi_{t, i}(\theta, r):=\phi_{i}(\theta, r / t) .
$$

Then there are $t$-uniform estimates as above. 
In particular, with respect to these diffeomorphisms, $\left(L, t^{2} g\right)$ is again a conifold. If $\rho$ is a radius function for $(L, g)$ then $t \rho$ is a radius function for $\left(L, t^{2} g\right)$.

Proof. Define the map

$$
\delta_{t}: \Sigma_{i} \times \mathbb{R}^{+} \rightarrow \Sigma_{i} \times \mathbb{R}^{+}, \quad(\theta, r) \mapsto(\theta, t r) .
$$

Since $\delta_{t}$ is simply a rescaling it preserves the Levi-Civita connection $\tilde{\nabla}$. Note that $\phi_{t, i}=\phi_{i} \circ \delta_{1 / t}$. It is simple to check that $\delta_{1 / t}^{*}\left(t^{2} \tilde{g}_{i}\right)=\tilde{g}_{i}$. Thus, for $r \geq t R$,

$$
\begin{aligned}
\left|\widetilde{\nabla}^{k}\left(\phi_{t, i}^{*}\left(t^{2} g\right)-\tilde{g}_{i}\right)\right|_{\tilde{g}_{i} \otimes \tilde{g}_{i}} & =\left|\widetilde{\nabla}^{k}\left(\delta_{1 / t}^{*} \phi_{i}^{*}\left(t^{2} g\right)-\tilde{g}_{i}\right)\right|_{\tilde{g}_{i} \otimes \tilde{g}_{i}} \\
& =\delta_{1 / t}^{*}\left(\left|\widetilde{\nabla}^{k}\left(\phi_{i}^{*}\left(t^{2} g\right)-t^{2} \tilde{g}_{i}\right)\right|_{\left.t^{2} \tilde{g}_{i} \otimes t^{2} \tilde{g}_{i}\right)}\right. \\
& =\delta_{1 / t}^{*}\left(\left|\widetilde{\nabla}^{k}\left(\phi_{i}^{*} g-\tilde{g}_{i}\right)\right|_{\left.t^{2} \tilde{g}_{i} \otimes \tilde{g}_{i}\right)}\right. \\
& \leq t^{-k} C_{k}(r / t)^{\nu_{i}-k}=C_{k}(r / t)^{\nu_{i}} r^{-k},
\end{aligned}
$$

where in the last line the factor $t^{-k}$ comes from measuring $\tilde{\nabla}^{k}$ using $t^{2} \tilde{g}_{i}$, cf. Remark 4.1. These inequalities can be rescaled as in Remark 6.3 to obtain the desired $t$-uniform estimates.

Now note that

$$
\phi_{t, i}^{*}(t \rho)_{\mid(\theta, r)}=t \rho \circ \phi_{t, i}(\theta, r)=t \rho \circ \phi_{i}(\theta, r / t)=t r / t=r
$$

so $t \rho$ is a radius function in the sense of Definition 6.6. CS ends can be studied analogously.

The following result is a direct consequence of Theorem 5.1 and Remark 5.2.

Corollary 6.11. Let $(L, g)$ be a conifold. Then, for all $t>0$ :

1) Choose a constant weight $\boldsymbol{\beta}$. Define weighted Sobolev spaces $W_{k, \boldsymbol{\beta}}^{p}$ as in Section 5 using the metric $t^{2} g$, the scale function $t \rho$ and the weight function $w:=(t \rho)^{-\boldsymbol{\beta}}$. Then all forms of the weighted Sobolev theorems hold for $\left(L, t^{2} g, t \rho,(t \rho)^{-\boldsymbol{\beta}}\right)$ with $t$-independent Sobolev constants.

2) More generally, let $\boldsymbol{\beta}$ be a function as in Definition 6.6. Choose a constant "reference" weight $\boldsymbol{\beta}^{\prime}$ and define weighted Sobolev spaces $W_{k, \boldsymbol{\beta}}^{p}$ as in Section 5 using the metric $t^{2} g$, the scale function $t \rho$ and the weight function $w_{t}:=\left(t^{\frac{\boldsymbol{\beta}^{\prime}-\boldsymbol{\beta}}{\boldsymbol{\beta}}} t \rho\right)^{-\boldsymbol{\beta}}$. Then the weighted norms $\|\cdot\|_{W_{k, \boldsymbol{\beta}}^{p}}$, 
calculated with respect to these choices, coincide with $t^{-\boldsymbol{\beta}^{\prime}}\|\cdot\|_{W_{k, \boldsymbol{\beta}}^{p}}$, calculated with respect to $\left(L, g, \rho, w:=\rho^{-\boldsymbol{\beta}}\right)$. In particular, all forms of the weighted Sobolev Embedding Theorems hold for $\left(L, t^{2} g, t \rho, w_{t}:=\right.$ $\left.\left(t^{\frac{\boldsymbol{\beta}^{\prime}-\boldsymbol{\beta}}{\boldsymbol{\beta}}} t \rho\right)^{-\boldsymbol{\beta}}\right)$ with $t$-independent Sobolev constants.

Remark 6.12. Compare the weights used in parts (1) and (2) above. Basically, to deal with variable weights we introduce a corrective factor of the form $t^{\boldsymbol{\beta}-\boldsymbol{\beta}^{\prime}}$ : since the exponent is bounded, for fixed $t$ this does not affect the decay/growth condition on the ends. Its effect is simply to yield estimates which are uniform with respect to $t$.

We conclude this section by summarizing the main definitions and properties of a second class of manifolds with ends, modelled on cylinders. We will see that the corresponding theory is closely related to that of conifolds.

Definition 6.13. Let $L$ be a manifold with ends. Let $g$ be a Riemannian metric on $L$. Choose an end $S_{i}$ with corresponding link $\Sigma_{i}$. We say that $S_{i}$ is an A. Cyl. end if the following conditions hold:

1) $\Sigma_{i}$ is endowed with a Riemannian metric $g_{i}^{\prime}$.

We then let $(\theta, z)$ denote the generic point on the product manifold $C_{i}:=\Sigma_{i} \times(-\infty, \infty)$ and $\tilde{h}_{i}:=d z^{2}+g_{i}^{\prime}$ denote the corresponding cylindrical metric on $C_{i}$.

2) There exist a constant $\nu_{i}<0$ and a diffeomorphism $\phi_{i}: \Sigma_{i} \times\left[R^{\prime}, \infty\right) \rightarrow$ $\overline{S_{i}}$ such that, as $z \rightarrow \infty$ and for all $k \geq 0$,

$$
\left|\widetilde{\nabla}^{k}\left(\phi_{i}^{*} g-\tilde{h}_{i}\right)\right|_{\tilde{h}_{i}}=O\left(\mathrm{e}^{\nu_{i} z}\right)
$$

where $\widetilde{\nabla}$ is the Levi-Civita connection on $C_{i}$ defined by $\tilde{h}_{i}$.

We say that $L$ is a A.Cyl. manifold if all ends are A. Cyl.

For the purposes of this paper the function spaces of most interest on A.Cyl. manifolds are not the ones already encountered, cf. Section 3 and Example 3.12. Instead, we use the following.

Definition 6.14. Let $(L, h)$ be a A.Cyl. manifold. We say that a smooth function $\zeta: L \rightarrow[1, \infty)$ is a radius function if $\phi_{i}^{*} \zeta=z$, where $\phi_{i}$ are the diffeomorphisms of Definition 6.2. Given any vector $\boldsymbol{\beta}=\left(\beta_{1}, \ldots, \beta_{e}\right) \in \mathbb{R}^{e}$, choose a function $\boldsymbol{\beta}$ on $L$ which, on each end $S_{i}$, restricts to the constant 
$\beta_{i}$. We call $(L, h, \zeta, \boldsymbol{\beta})$ a weighted A. Cyl. manifold. Given any metric pair $(E, \nabla)$ we define Banach spaces of sections of $E$ in the following two ways. The weighted spaces of $C^{k}$ sections of $E$ are defined by

$$
C_{\boldsymbol{\beta}}^{k}(E):=\left\{\sigma \in C^{k}(E):\|\sigma\|_{C_{\boldsymbol{\beta}}^{k}}<\infty\right\}
$$

where we use the norm $\|\sigma\|_{C_{\beta}^{k}}:=\sum_{j=0}^{k} \sup _{x \in L}\left|\mathrm{e}^{-\boldsymbol{\beta}(x) \zeta(x)} \nabla^{j} \sigma\right|$.

The weighted Sobolev spaces are defined by

$$
\begin{gathered}
W_{k, \boldsymbol{\beta}}^{p}(E):=\text { Banach space completion of the space } \\
\left\{\sigma \in C^{\infty}(E):\|\sigma\|_{W_{k, \boldsymbol{\beta}}^{p}}<\infty\right\}
\end{gathered}
$$

where $p \in[1, \infty), \quad k \geq 0$ and we use the norm $\|\sigma\|_{W_{k, \boldsymbol{\beta}}^{p}}:=\left(\sum_{j=0}^{k}\right.$ $\left.\int_{L}\left|\mathrm{e}^{-\boldsymbol{\beta} \zeta} \nabla^{j} \sigma\right|^{p} \operatorname{vol}_{h}\right)^{1 / p}$.

Both types of spaces are independent of the particular choices made.

Remark 6.15. It is simple to see that the norm $\|\sigma\|_{W_{k, \boldsymbol{\beta}}^{p}}$ is equivalent to the norm defined by $\sum_{j=0}^{k}\left(\int_{L}\left|\nabla^{j}\left(\mathrm{e}^{-\boldsymbol{\beta} \zeta} \sigma\right)\right|^{p} \operatorname{vol}_{h}\right)^{1 / p}$. This leads to the following fact.

Let $W_{k}^{p}(E)$ denote the standard Sobolev spaces for $(L, h)$ introduced in Section 3. Let $\mathrm{e}^{\boldsymbol{\beta} \zeta} \cdot W_{k}^{p}$ denote the space of all sections of $E$ of the form $\sigma=\mathrm{e}^{\boldsymbol{\beta} \zeta} \tau$ for some $\tau \in W_{k}^{p}(E)$, endowed with the norm $\|\sigma\|:=\|\tau\|$. Then $W_{k, \boldsymbol{\beta}}^{p}(E)=\mathrm{e}^{\boldsymbol{\beta} \zeta} \cdot W_{k}^{p}(E)$ as sets and the norms are equivalent. Analogously, the spaces $C_{\boldsymbol{\beta}}^{k}(E)$ are equivalent to the spaces $\mathrm{e}^{\boldsymbol{\beta} \zeta} \cdot C^{k}(E)$, where $C^{k}(E)$ are the standard spaces of $C^{k}$ sections used in Section 3.

As before, weighted spaces defined with respect to A.Cyl. metrics and cylindrical metrics are equivalent. Remark 6.15 allows us to reduce the weighted Sobolev Embedding Theorems for A.Cyl. manifolds to the standard Sobolev Embedding Theorems, obtaining results analogous to Corollary 6.8 and Remark 6.9. According to [4] Theorem 3.1 and Proposition 3.2, the spaces $C_{c}^{\infty}$ are dense in the standard Sobolev spaces defined for manifolds whose ends are exactly cylindrical. The same is then true for weighted Sobolev spaces on A.Cyl. manifolds.

Remark 6.16. It is interesting to compare Definitions 6.14 and 6.6. Assume $(L, h)$ is an A.Cyl. manifold with respect to certain diffeomorphisms $\phi_{i}=$ $\phi_{i}(\theta, z)$ as in Definition 6.2. Since the corresponding weighted Sobolev spaces 
are equivalent we may assume that $h$ is exactly cylindrical on each end, i.e., using the notation of Definition 6.2 it can be written $h=d z^{2}+g_{i}^{\prime}$. Consider the conformally rescaled metric $g:=\mathrm{e}^{2 \zeta} h$. Using the change of variables $r=\mathrm{e}^{z}$ it is simple to check that $g=d r^{2}+r^{2} g_{i}^{\prime}$. This implies that $(L, g)$ is an $\mathrm{AC}$ manifold with respect to the diffeomorphisms $\phi_{i}(\theta, \log z)$. Viceversa, any $\mathrm{AC}$ metric on $L$ defines a conformally equivalent A.Cyl. metric. Note that if $z \in\left(R^{\prime}, \infty\right)$ then $r \in(R, \infty)$ with $R:=e^{R^{\prime}}$ and that $r^{-m} \operatorname{vol}_{g}=\operatorname{vol}_{h}$. Thus, by change of variables,

$$
\int_{R}^{\infty} \int_{\Sigma}\left|r^{-\boldsymbol{\beta}} \sigma\right|^{p} r^{-m} \operatorname{vol}_{g}=\int_{R^{\prime}}^{\infty} \int_{\Sigma}\left|\mathrm{e}^{-\boldsymbol{\beta} z} \sigma\right|^{p} \operatorname{vol}_{h} .
$$

This shows that the spaces $L_{\boldsymbol{\beta}}^{p}(E)$ of sections of $E$ coincide for $(L, g)$ and $(L, h)$, while the corresponding norms are equivalent (but again, as in Remark 4.1, one may need to take into account, which metric is being used on $E$ in the two cases).

The same is true also for Sobolev spaces of higher order. Specifically, an explicit calculation shows that the Levi-Civita connections defined by $h$ and $g$ are equivalent, i.e., the corresponding Christoffel symbols coincide up to constant multiplicative factors. It thus makes no difference which metric is used to define $\nabla$. On the other hand, the norm inside the integral does depend on the choice of metric. For example,

$$
\int_{R}^{\infty} \int_{\Sigma}\left|r^{-\boldsymbol{\beta}+j} \nabla^{j} f \sigma\right|_{g}^{p} r^{-m} \operatorname{vol}_{g}=\int_{R^{\prime}}^{\infty} \int_{\Sigma}\left|\mathrm{e}^{-\boldsymbol{\beta} z} \nabla^{j} \sigma\right|_{h}^{p} \operatorname{vol}_{h}
$$

This proves that the spaces $W_{k, \boldsymbol{\beta}}^{p}(E)$ are equivalent.

Analogous results hold for CS manifolds: if $h$ is A.Cyl. then $g:=\mathrm{e}^{-2 \zeta} h$ is CS. In this case

$$
\int_{0}^{\epsilon} \int_{\Sigma}\left|r^{-\boldsymbol{\beta}} f\right|^{p} r^{-m} \operatorname{vol}_{g}=\int_{-\log \epsilon}^{\infty} \int_{\Sigma}\left|e^{\boldsymbol{\beta} z} f\right|^{p} \operatorname{vol}_{h}
$$

so the space $L_{\boldsymbol{\beta}}^{p}$ for $(L, g)$ coincides with the space $L_{-\boldsymbol{\beta}}^{p}$ for $(L, h)$.

These facts show, for example, that the Sobolev Embedding Theorems for conifolds and A.Cyl. manifolds are simply two different points of view on the same result. They also show that $C_{c}^{\infty}$ is dense in all weighted Sobolev spaces on conifolds because, as already seen, this is true on A.Cyl. manifolds. Finally, they show that in Remark 6.3, we are really using the cylindrical metric $r^{-2} \tilde{g}=\tilde{h}$ to "measure" $\widetilde{\nabla}^{k}$ (in the sense of Remark 4.1). 


\section{Part 2. Elliptic estimates}

We now turn to the theory of elliptic operators via weighted Sobolev spaces, focusing on Fredholm and index results for the manifolds discussed in Section 6. Results of this kind have been proved by various authors, e.g., Lockhart-McOwen [11], Lockhart [10] and Melrose [13]. We will follow the point of view of Lockhart and $\mathrm{McO}$ wen to which we refer for details, see also Joyce-Salur [8].

\section{Fredholm results for elliptic operators on A.Cyl. manifolds}

We start with the case of A.Cyl. manifolds. The theory requires appropriate assumptions on the asymptotic behaviour of the operators, which we roughly summarize as follows.

Definition 7.1. Given a manifold $\Sigma$, consider the projection $\pi: \Sigma \times \mathbb{R} \rightarrow$ $\Sigma$. A vector bundle $E_{\infty}$ on $\Sigma \times \mathbb{R}$ is translation-invariant if it is of the form $\pi^{*} E^{\prime}$, for some vector bundle $E^{\prime}$ over $\Sigma$. We define the notion of translationinvariant metrics and connections analogously.

Let $P_{\infty}: C^{\infty}\left(E_{\infty}\right) \rightarrow C^{\infty}\left(F_{\infty}\right)$ be a differential operator between translation-invariant vector bundles. We say that $P_{\infty}$ is translation-invariant if it commutes with the action of $\mathbb{R}$ on $\Sigma \times \mathbb{R}$ determined by translations; equivalently, writing $P_{\infty}=\sum A_{k}^{\infty} \cdot \nabla^{k}$ with respect to a translationinvariant $\nabla$, if the coefficient tensors $A_{k}^{\infty}$ are independent of $z$.

Let $(L, h)$ be an A.Cyl. manifold with link $\Sigma=\amalg \Sigma_{i}$. Let $E, F$ be vector bundles over $L$. Assume there exist translation-invariant vector bundles $E_{\infty}$, $F_{\infty}$ over $\Sigma \times \mathbb{R}$ such that, using the notation of Definition $6.13, \phi_{i}^{*}\left(E_{\mid S_{i}}\right)$ (respectively, $\phi_{i}^{*}\left(F_{\mid S_{i}}\right)$ ) coincides with the restriction to $\Sigma_{i} \times\left(R^{\prime}, \infty\right)$ of $E_{\infty}$ (respectively, $F_{\infty}$ ).

Let $P_{\infty}=\sum A_{k}^{\infty} \cdot \nabla^{k}: C^{\infty}\left(E_{\infty}\right) \rightarrow C^{\infty}\left(F_{\infty}\right)$ be a translation-invariant linear differential operator of order $n$. Consider a linear operator $P$ : $C^{\infty}(E) \rightarrow C^{\infty}(F)$. We say that $P$ is asymptotic to $P_{\infty}$ if on each end there exists $\nu_{i}<0$ such that, writing $P=\sum A_{k} \cdot \nabla^{k}$ (up to identifications) and as $z \rightarrow \infty$,

$$
\left|\nabla^{j}\left(A_{k}-A_{k}^{\infty}\right)\right|=O\left(\mathrm{e}^{\nu_{i} z}\right),
$$

where $|\cdot|$ is defined by the translation-invariant metrics. We call $\nu_{i}$ the convergence rates of the operator $P$. 
In what follows, to define the spaces $W_{k, \boldsymbol{\beta}}^{p}(E)$, we will assume that $E$ is endowed with a metric and a metric connection which are asymptotic to the translation-invariant data on $E_{\infty}$, in the appropriate sense.

Assume $P$ is a linear operator of order $n$ with bounded coefficients $A_{k}$. It follows from Definition 6.14 that, for all $p>1, k \geq 0$ and $\boldsymbol{\beta}, P$ extends to a continuous map

$$
P: W_{k+n, \boldsymbol{\beta}}^{p}(E) \rightarrow W_{k, \boldsymbol{\beta}}^{p}(F) .
$$

Remark 7.2. It will sometimes be useful to denote by $P_{\boldsymbol{\beta}}$ the extended operator of Equation (7.1), so as to emphasize the particular weight being used.

Now assume $P$ is asymptotic to a translation-invariant operator $P_{\infty}$. Then Equation (7.1) holds also for the operator $\mathrm{e}^{-\boldsymbol{\nu} \zeta}\left(P-P_{\infty}\right)$, where $\boldsymbol{\nu}<0$ denotes the convergence rates of $P$ as in Definition 7.1. This implies that the operator $P-P_{\infty}$ extends to a continuous map

$$
P-P_{\infty}: W_{k+n, \boldsymbol{\beta}}^{p}(E) \rightarrow W_{k, \boldsymbol{\beta}+\boldsymbol{\nu}}^{p}(F) .
$$

Note that if $\boldsymbol{\beta}<\boldsymbol{\beta}^{\prime}$ then $W_{k+n, \boldsymbol{\beta}}^{p}(E) \subset W_{k+n, \boldsymbol{\beta}^{\prime}}^{p}(E)$ and that the operator $P_{\boldsymbol{\beta}^{\prime}}$ extends the operator $P_{\boldsymbol{\beta}}$. Note also that $C_{c}^{\infty}(E) \subset W_{k, \boldsymbol{\beta}}^{p}(E)$ as a dense subset. Dualizing this relation allows us to identify the dual space $\left(W_{k, \boldsymbol{\beta}}^{p}(E)\right)^{*}$ with a subspace of the space of distributions $\left(C_{c}^{\infty}(E)\right)^{*}$. It is customary to denote this space $W_{-k,-\boldsymbol{\beta}}^{p^{\prime}}(E)$. Endowed with the appropriate norm, it again contains $C_{c}^{\infty}(E)$ as a dense subset. The duality map $W_{-k,-\boldsymbol{\beta}}^{p^{\prime}}(E) \times W_{k, \boldsymbol{\beta}}^{p}(E) \rightarrow \mathbb{R}$, restricted to this subset, coincides with the map

$$
C_{c}^{\infty}(E) \times W_{k, \boldsymbol{\beta}}^{p}(E) \rightarrow \mathbb{R}, \quad<\sigma, \sigma^{\prime}>:=\int_{L}\left(\sigma, \sigma^{\prime}\right)_{E} \operatorname{vol}_{h} .
$$

This map extends by continuity to a map defined on $W_{l,-\boldsymbol{\beta}}^{p^{\prime}}(E) \times W_{k, \boldsymbol{\beta}}^{p}(E)$ for all $l \geq 0$, showing that $W_{-k,-\boldsymbol{\beta}}^{p^{\prime}}(E)$ also contains all spaces $W_{l,-\boldsymbol{\beta}}^{p^{\prime}}(E)$. It can be shown that $P$ admits continuous extensions as in Equation (7.1) for any $k \in \mathbb{Z}$.

Lemma 7.3. Let $P: C^{\infty}(E) \rightarrow C^{\infty}(F)$ be a linear differential operator of order $n$, asymptotic to a translation-invariant operator $P_{\infty}$. Let $P^{*}$ : 
$C^{\infty}(F) \rightarrow C^{\infty}(E)$ denote its formal adjoint. Consider the continuous extension of $P^{*}$ to the spaces

$$
P^{*}: W_{-k,-\boldsymbol{\beta}}^{p^{\prime}}(F) \rightarrow W_{-k-n,-\boldsymbol{\beta}}^{p^{\prime}}(E) .
$$

Under the identification of Sobolev spaces of negative order with dual spaces, this operator coincides with the operator dual to that of Equation (7.1),

$$
P^{*}:\left(W_{k, \boldsymbol{\beta}}^{p}(F)\right)^{*} \rightarrow\left(W_{k+n, \boldsymbol{\beta}}^{p}(E)\right)^{*} .
$$

Furthermore if $E=F$ and $P$ is self-adjoint, i.e., $P=P^{*}$ on smooth compactly-supported sections, then $P=P^{*}$ on any space $W_{k, \boldsymbol{\beta}}^{p}$.

Proof. The formal adjoint of $P$ is asymptotic to the formal adjoint of $P_{\infty}$, so the extensions exist as specified. The statement of this lemma can be clarified by adopting the notation of Remark 7.2 : the claim is then that $\left(P^{*}\right)_{-\boldsymbol{\beta}}=$ $\left(P_{\boldsymbol{\beta}}\right)^{*}$, where on the left the superscript $*$ denotes the formal adjoint and on the right it denotes the dual map.

Since both maps are continuous, it is sufficient to show that they coincide on a dense subset: in particular that $\left(P^{*}\right)_{-\boldsymbol{\beta}}(\tau)=\left(P_{\boldsymbol{\beta}}\right)^{*}(\tau)$, for all $\tau \in C_{c}^{\infty}(F)$. Since we are identifying $\left(P^{*}\right)_{-\beta}(\tau)$ with an element of the dual space $\left(W_{k+n, \boldsymbol{\beta}}^{p}(E)\right)^{*}$, we can again invoke continuity to claim that it is sufficient to prove that, for all $e \in C_{c}^{\infty}(E)$,

$$
\left\langle\left(P^{*}\right)_{-\boldsymbol{\beta}}(\tau), e\right\rangle=\left\langle\left(P_{\boldsymbol{\beta}}\right)^{*}(\tau), e\right\rangle .
$$

This claim is now a direct consequence of the definitions and of Equation (7.3).

The claim concerning self-adjoint operators is a simple consequence of continuity.

Remark 7.4. As already remarked, $\boldsymbol{\beta}^{\prime}>\boldsymbol{\beta}$ implies $P_{\boldsymbol{\beta}^{\prime}}$ extends $P_{\boldsymbol{\beta}}$. This shows that the spaces $\operatorname{Ker}\left(P_{\boldsymbol{\beta}}\right)$ grow with $\boldsymbol{\beta}$. On the other hand, as a vector space, the cokernel of $P$ in Equation (7.1) is not canonically a subspace of $W_{k, \boldsymbol{\beta}}^{p}(F)$ so there is no canonical way of relating cokernels corresponding to different weights. However, consider the following construction, for which we assume $P, P^{*}$ are Fredholm. Pick $\tau_{1} \in W_{k, \boldsymbol{\beta}}^{p}(F)$ such that $\left\langle\sigma, \tau_{1}\right\rangle \neq 0$, for some $\sigma \in \operatorname{Ker}\left(P^{*}\right)$. According to Remark 2.3 this implies that $\tau_{1}$ does not belong to $\operatorname{Im}(P)$. By density we can then find $\tilde{\tau_{1}}$ which is smooth and compactly-supported and does not belong to $\operatorname{Im}(P)$. Now choose $\tau_{2}$ satisfy$\operatorname{ing}\left\langle\sigma, \tau_{2}\right\rangle \neq 0$ for some $\sigma \in \operatorname{Ker}\left(P^{*}\right)$ and which is linearly independent of $\tau_{1}$, 
etc. After a finite number of steps we will have found a vector space spanned by $\tilde{\tau_{1}}, \ldots, \tilde{\tau_{k}}$ which defines a complement to $\operatorname{Im}(P)$ and thus is isomorphic to $\operatorname{Coker}(P)$. Note that by construction $\tilde{\tau}_{i}$ belong to all spaces $W_{k, \boldsymbol{\beta}}^{p}(F)$. On the other hand, as $\boldsymbol{\beta}$ decreases the dual weight $-\boldsymbol{\beta}$ increases, so $\operatorname{Ker}\left(P^{*}\right)$ increases, so the $\tilde{\tau}_{i}$ chosen for the weight $\boldsymbol{\beta}$ can be used also for any weight $\boldsymbol{\beta}^{\prime}<\boldsymbol{\beta}$. The conclusion is that we can construct spaces representing the cokernel, which grow as $\boldsymbol{\beta}$ decreases, i.e., as the function spaces become smaller.

Now assume $P$ is elliptic. We are interested in conditions ensuring that the extended map of Equation (7.1) is Fredholm.

Definition 7.5. Let $\Sigma$ be a compact oriented Riemannian manifold with connected components $\Sigma_{1}, \ldots, \Sigma_{e}$. Let $P_{\infty}$ be a translation-invariant operator on $\Sigma \times \mathbb{R}$. Consider the complexified operator $P_{\infty}: E_{\infty} \otimes \mathbb{C} \rightarrow F_{\infty} \otimes \mathbb{C}$. Choose a connected component $\Sigma_{j} \times \mathbb{R}$ and fix $\gamma+i \delta \in \mathbb{C}$. Let us restrict our attention to the space of sections of $E_{\infty} \otimes \mathbb{C}$ of the form $\mathrm{e}^{(\gamma+i \delta) z} \sigma(\theta)$. Consider the subspace $V_{\gamma+i \delta}^{j}$ determined by the solutions to the problem $P_{\infty}\left(\mathrm{e}^{(\gamma+i \delta) z} \sigma(\theta)\right)=0$ on $\Sigma_{j} \times \mathbb{R}$. We define the space $\mathcal{C}_{P_{\infty}}^{j} \subseteq \mathbb{C}$ to be the space of all $\gamma+i \delta$ such that $V_{\gamma+i \delta}^{j} \neq 0$. We then define the space of exceptional weights for $P_{\infty}$ on $\Sigma_{j} \times \mathbb{R}$ to be the corresponding set of real values, $\mathcal{D}_{P_{\infty}}^{j}:=\operatorname{Re}\left(\mathcal{C}_{P_{\infty}}^{j}\right) \subseteq \mathbb{R}$.

Now fix a multi-index $\gamma+i \boldsymbol{\delta} \in \mathbb{C}^{e}$. Let $V_{\gamma+i \boldsymbol{\delta}}:=\oplus_{j=1}^{e} V_{\gamma_{j}+i \delta_{j}}^{j}$. We define the space of exceptional weights for $P_{\infty}$ on $\Sigma \times \mathbb{R}$, denoted $\mathcal{D}_{P_{\infty}} \subseteq \mathbb{R}^{e}$, to be the set of multi-indices $\gamma=\left(\gamma_{1}, \ldots, \gamma_{e}\right)$ such that, for some $j, \gamma_{j} \in \mathcal{D}_{P_{\infty}}^{j}$.

Remark 7.6. Definition 7.5 introduces the exceptional weights via the kernel of $P_{\infty}$ and the space of sections with exponential growth. Along the lines of [11], the exceptional weights can equivalently be defined as follows. Separating the $\partial \theta$ derivatives from the $\partial z$ derivatives and setting $D z=$ $-i \partial z$, we can write

$$
P_{\infty}=\sum A_{k}(\theta, \partial \theta)(\partial z)^{k}=\sum A_{k}(\theta, \partial \theta) i^{k}(D z)^{k},
$$

where, to simplify the notation, $\partial \theta$ denotes any combination of derivatives in the $\theta$ variables. For any $\lambda \in \mathbb{C}$, set $P_{\lambda}:=\sum A_{k}(\theta, \partial \theta) i^{k} \lambda^{k}$. Note that

$$
P_{\infty}\left(\mathrm{e}^{i \lambda z} \sigma(\theta)\right)=\sum A_{k}(\theta, \partial \theta)(i \lambda)^{k} \sigma \mathrm{e}^{i \lambda z}=\left(P_{\lambda}(\sigma)\right) \mathrm{e}^{i \lambda z}
$$

so $P_{\infty}\left(\mathrm{e}^{i \lambda z} \sigma(\theta)\right)=0$ iff $P_{\lambda}(\sigma)=0$. We view the latter as a generalized eigenvalue problem on $\Sigma$ and say that $\lambda$ is an eigenvalue iff the corresponding 
generalized eigenvalue problem admits non-trivial solutions. It follows from the above calculations that a weight $\gamma \in \mathbb{R}$ is exceptional in the sense of Definition 7.5 iff $-\gamma=\operatorname{Im}(\lambda)$, for some eigenvalue $\lambda$.

For elliptic operators it turns out that the exceptional weights of $P_{\infty}$ determine the possible Fredholm extensions of any $P$ asymptotic to $P_{\infty}$.

Theorem 7.7. Let $(L, h)$ be an A.Cyl. manifold with link $\Sigma=\amalg \Sigma_{i}$. Let $P: C^{\infty}(E) \rightarrow C^{\infty}(F)$ be a linear elliptic operator of order $n$, asymptotic to an elliptic operator $P_{\infty}$.

Then each $\mathcal{D}_{P_{\infty}}^{j}$ is discrete in $\mathbb{R}$ so $\mathcal{D}_{P_{\infty}}$ defines a discrete set of hyperplanes in $\mathbb{R}^{e}$. Furthermore, for each $p>1$ and $k \geq 0$, the extended operator $P_{\boldsymbol{\gamma}}: W_{k+n, \boldsymbol{\gamma}}^{p}(E) \rightarrow W_{k, \boldsymbol{\gamma}}^{p}(F)$ is Fredholm iff $\boldsymbol{\gamma} \notin \mathcal{D}_{P_{\infty}}$.

In a similar vein, we can compute how the index of $P$ depends on $\gamma$.

Definition 7.8. Consider the complexified operator $P_{\infty}: E_{\infty} \otimes \mathbb{C} \rightarrow$ $F_{\infty} \otimes \mathbb{C}$. Choose a connected component $\Sigma_{j} \times \mathbb{R}$ of $\Sigma \times \mathbb{R}$ and fix $\gamma+i \delta \in \mathcal{C}_{P_{\infty}}^{j}$. We denote by $\widetilde{V}_{\gamma+i \delta}^{j}$ the space of solutions to the problem $P_{\infty}\left(e^{(\gamma+i \delta) z} \sigma(\theta, z)\right)=0$ on $\Sigma_{j} \times \mathbb{R}$, where $\sigma(\theta, z)$ is polynomial in $z$. We can extend this definition to all $\gamma+i \delta$ by setting $\widetilde{V}_{\gamma+i \delta}^{j}=\{0\}$ if $\gamma+i \delta \notin \mathcal{C}_{P_{\infty}}^{j}$. Note that $V_{\gamma+i \delta}^{j} \leq \widetilde{V}_{\gamma+i \delta}^{j}$. Given any $\gamma \in \mathbb{R}$ we now set $\widetilde{V}_{\gamma}^{j}:=\bigoplus_{\delta \in \mathbb{R}} \widetilde{V}_{\gamma+i \delta}^{j}$, then define the multiplicity of $\gamma$ on $\Sigma_{j} \times \mathbb{R}$ by $m_{P_{\infty}}^{j}(\gamma):=\operatorname{dim}\left(\widetilde{V}_{\gamma}^{j}\right)$.

Now fix a multi-index $\gamma \in \mathbb{R}^{e}$. We define the multiplicity of $\gamma$ on $\Sigma \times \mathbb{R}$ to be $m_{P_{\infty}}(\gamma):=\sum_{j=1}^{e} m_{P_{\infty}}^{j}\left(\gamma_{j}\right)$.

Theorem 7.9. In the setting of Theorem 7.7, each multiplicity $m_{P_{\infty}}(\gamma)$ is finite. Furthermore, choose $\gamma_{1}, \gamma_{2} \in \mathbb{R}^{e} \backslash \mathcal{D}_{P_{\infty}}$ with $\gamma_{1} \leq \gamma_{2}$. Then

$$
i_{\boldsymbol{\gamma}_{2}}(P)-i_{\boldsymbol{\gamma}_{1}}(P)=\sum_{\boldsymbol{\gamma} \in \mathcal{D}_{P_{\infty}, \boldsymbol{\gamma}_{1} \leq \boldsymbol{\gamma} \leq \boldsymbol{\gamma}_{2}}} m_{P_{\infty}}(\boldsymbol{\gamma})
$$

Remark 7.10. Assume we can compute the value of $i_{\boldsymbol{\gamma}}(P)$ for a specific good choice of non-exceptional $\gamma$. Theorem 7.9 then allows us to compute $i_{\gamma}(P)$ for all non-exceptional $\gamma$ in terms of data on the link.

The following result is proved in [11] Section 7, cf. also [8], as a consequence of the Sobolev embedding and change of index theorems.

Proposition 7.11. In the setting of Theorem 7.9, assume $\gamma$ and $\gamma^{\prime}$ belong to the same connected component of $\mathbb{R}^{e} \backslash \mathcal{D}_{P_{\infty}}$. Then $i_{\boldsymbol{\gamma}}(P)=i_{\boldsymbol{\gamma}^{\prime}}(P)$ and 
$\operatorname{Ker}\left(P_{\boldsymbol{\gamma}}\right)=\operatorname{Ker}\left(P_{\boldsymbol{\gamma}^{\prime}}\right)$. Furthermore, the index and kernel are independent of the choice of $p$ and $k$.

Example 7.12. Assume $(L, h)$ is an A.Cyl. manifold with one end with link $\left(\Sigma, g^{\prime}\right)$. Let $P:=\Delta_{h}$ denote the positive Laplace operator on functions. Then $P$ is asymptotic to the Laplace operator $\Delta_{\tilde{h}}$ defined on the product $\left(\Sigma \times \mathbb{R}, \tilde{h}:=d z^{2}+g^{\prime}\right)$. One can check that $\Delta_{\tilde{h}}=-(\partial z)^{2}+\Delta_{g^{\prime}}$ and that $\Delta_{\tilde{h}} \mathrm{e}^{(\gamma+i \delta) z} \sigma(\theta)=0$ iff $\delta=0$ and $\Delta_{g^{\prime}} \sigma=\gamma^{2} \sigma$. In other words, the harmonic functions on the cylinder which have exponential growth are generated by the eigenvalues of $\Delta_{g^{\prime}}$. In particular, the exceptional weights for $\Delta_{h}$ are of the form $\pm \sqrt{e_{n}}$, where $e_{n}$ are the eigenvalues of $\Delta_{g^{\prime}}$.

\section{Weight-crossing}

Let $(L, h)$ be an A.Cyl. manifold. Let $P: C^{\infty}(E) \rightarrow C^{\infty}(F)$ be a linear elliptic operator asymptotic to some $P_{\infty}$ as in Definition 7.1. Consider the extension of $P$ to weighted Sobolev spaces as in Equation (7.1). When $\boldsymbol{\beta}$ changes value crossing an exceptional weight the change of index formula given in Theorem 7.9 leads us to expect that the kernel and/or cokernel of $P$ will change. Specifically, when $\boldsymbol{\beta}$ increases, we expect the kernel of $P$ to increase and the cokernel to decrease. The process by which this occurs can be formalized using the Fredholm and index results stated in Section 7. The notation we rely on was introduced in Definitions 7.5 and 7.8. To simplify the notation, throughout this section we forgo the distinction between bundles (or operators) and their complexifications.

Literally speaking, given any index $\gamma \in \mathbb{R}$ and end $S_{j}$, the sections in each $\widetilde{V}_{\gamma}^{j}$ are defined on $\Sigma_{j} \times \mathbb{R}$. Using the identification $\phi_{j}$, we can alternatively think of them as being defined on $S_{j}$. However, we can also think of them as being globally defined on $L$ by first choosing a basis of sections $\sigma_{i}^{j}$ for each $\widetilde{V}_{\gamma}^{j}$, then interpolating between them so as to get smooth extensions $\sigma_{i}^{j}$ over $L$. In particular, it may be useful to choose the extension of each $\sigma_{i}^{j}$ so that it is identically zero on the other ends. The construction implies that each $P_{\infty}\left(\sigma_{i}^{j}\right)$ has compact support. By choosing the extensions generically over $L \backslash S$, we can assume that all $P\left(\sigma_{i}^{j}\right)$ are linearly independent. This implies that $P$ is injective on $\widetilde{V}_{\boldsymbol{\gamma}}$

Now assume $\gamma \in \mathbb{R}^{e}$ is exceptional. Then, for any $\boldsymbol{\nu}<0$ with $|\boldsymbol{\nu}|<<1$,

$$
P: W_{k+n, \boldsymbol{\gamma}+\boldsymbol{\nu}}^{p}(E) \rightarrow W_{k, \boldsymbol{\gamma}+\boldsymbol{\nu}}^{p}(F)
$$


is Fredholm. In particular, let $\boldsymbol{\nu}<0$ be the convergence rates of $P$ as in Definition 7.1. We will assume that $|\boldsymbol{\nu}|<<1$ as above. Writing $P(\sigma)=$ $\left(P-P_{\infty}\right)(\sigma)+P_{\infty}(\sigma)$ and using Equation (7.2) then shows that $P\left(\widetilde{V}_{\boldsymbol{\gamma}}\right) \subset$ $W_{k, \boldsymbol{\gamma}+\boldsymbol{\nu}}^{p}(F)$. Since $P$ is injective on $\widetilde{V}_{\boldsymbol{\gamma}}$ we can define a decomposition

$$
\tilde{V}_{\gamma}=\widetilde{V}_{\gamma}^{\prime} \oplus \tilde{V}_{\gamma}^{\prime \prime}
$$

by defining $P\left(\widetilde{V}_{\boldsymbol{\gamma}}^{\prime}\right):=P\left(\widetilde{V}_{\boldsymbol{\gamma}}\right) \cap \operatorname{Im}\left(P_{\boldsymbol{\gamma}+\boldsymbol{\nu}}\right)$ and choosing any complement $\widetilde{V}_{\boldsymbol{\gamma}}^{\prime \prime}$. By definition, $P\left(\widetilde{V}_{\boldsymbol{\gamma}}^{\prime \prime}\right) \cap \operatorname{Im}\left(P_{\boldsymbol{\gamma}+\boldsymbol{\nu}}\right)=0$. In other words, we can think of $P\left(\widetilde{V}_{\boldsymbol{\gamma}}^{\prime \prime}\right)$ as belonging to the cokernel of $P_{\boldsymbol{\gamma}+\boldsymbol{\nu}}$. On the other hand, $P\left(\widetilde{V}_{\boldsymbol{\gamma}}^{\prime \prime}\right)$ belongs to the image of $P_{\boldsymbol{\gamma}-\boldsymbol{\nu}}$ because $\widetilde{V}_{\boldsymbol{\gamma}} \subset W_{k+n, \boldsymbol{\gamma}-\boldsymbol{\nu}}^{p}(E)$. Roughly speaking, $P\left(\widetilde{V}_{\boldsymbol{\gamma}}^{\prime \prime}\right)$ thus describes the portion of the cokernel of $P$ which "disappears" when crossing the exceptional weight $\gamma$.

By construction, for any $\sigma \in \widetilde{V}_{\boldsymbol{\gamma}}^{\prime}$ there exists $u_{\sigma} \in W_{k+n, \boldsymbol{\gamma}+\boldsymbol{\nu}}^{p}(E)$ such that $P(\sigma)=P\left(u_{\sigma}\right)$. Note that $u_{\sigma}$ is not necessarily uniquely defined. However, it is sufficient to fix a choice of $u_{\sigma}$ for each element of a basis of $\widetilde{V}_{\boldsymbol{\gamma}}^{\prime}$ to obtain a unique choice of $u_{\sigma}$ for any $\sigma \in \widetilde{V}_{\boldsymbol{\gamma}}^{\prime}$. Note also that $\sigma-u_{\sigma} \in$ $W_{k+n, \boldsymbol{\gamma}-\boldsymbol{\nu}}^{p}(E)$. We have thus defined a map

$$
\widetilde{V}_{\boldsymbol{\gamma}}^{\prime} \rightarrow \operatorname{Ker}\left(P_{\boldsymbol{\gamma}-\boldsymbol{\nu}}\right), \quad \sigma \mapsto \sigma-u_{\sigma} \notin W_{k+n, \boldsymbol{\gamma}+\boldsymbol{\nu}}^{p}(E) .
$$

The image of the map of Equation (8.3) thus defines a space of "new" elements in $\operatorname{Ker}(P)$, generated by crossing the exceptional weight $\boldsymbol{\gamma}$. Note that $u_{\sigma}$ is of strictly lower order of growth compared to $\sigma$. This shows that the map of Equation (8.3) is injective and that the elements in its image admit an asymptotic expansion of the form $e^{\boldsymbol{\gamma} \zeta}+$ lower order. The following result shows that every new element in $\operatorname{Ker}(P)$ arises this way.

Lemma 8.1. Let us identify $\widetilde{V}_{\boldsymbol{\gamma}}^{\prime}$ with its image under the map of Equation (8.3). Then

$$
\operatorname{Ker}\left(P_{\boldsymbol{\gamma}-\boldsymbol{\nu}}\right)=\operatorname{Ker}\left(P_{\boldsymbol{\gamma}+\boldsymbol{\nu}}\right) \oplus \tilde{V}_{\boldsymbol{\gamma}}^{\prime}
$$

Proof. By injectivity, the inequality $\supseteq$ is clear. To prove the lemma it is thus sufficient to prove that the inverse inequality holds on the corresponding dimensions. Choose any $\sigma \in \widetilde{V}_{\boldsymbol{\gamma}}^{\prime \prime}$. According to Remark 2.3,

$$
\begin{aligned}
& P(\sigma) \in \operatorname{Im}\left(P_{\boldsymbol{\gamma}-\boldsymbol{\nu}}\right) \Leftrightarrow\langle\tau, P(\sigma)\rangle=0, \forall \tau \in \operatorname{Ker}\left(P_{-\boldsymbol{\gamma}+\boldsymbol{\nu}}^{*}\right) \\
& P(\sigma) \in \operatorname{Im}\left(P_{\boldsymbol{\gamma}+\boldsymbol{\nu}}\right) \Leftrightarrow\langle\tau, P(\sigma)\rangle=0, \forall \tau \in \operatorname{Ker}\left(P_{-\boldsymbol{\gamma}-\boldsymbol{\nu}}^{*}\right) .
\end{aligned}
$$


From the definition of $\widetilde{V}_{\boldsymbol{\gamma}}^{\prime \prime}$ we know that $P(\sigma) \in \operatorname{Im}\left(P_{\boldsymbol{\gamma}-\boldsymbol{\nu}}\right)$ and that $P(\sigma) \notin$ $\operatorname{Im}\left(P_{\boldsymbol{\gamma}+\boldsymbol{\nu}}\right)$ unless $\sigma=0$. Note also that $\operatorname{Ker}\left(P_{-\boldsymbol{\gamma}+\boldsymbol{\nu}}^{*}\right) \subseteq \operatorname{Ker}\left(P_{-\boldsymbol{\gamma}-\boldsymbol{\nu}}^{*}\right)$. We conclude that the following map is well-defined:

$$
\frac{\operatorname{Ker}\left(P_{-\boldsymbol{\gamma}-\boldsymbol{\nu}}^{*}\right)}{\operatorname{Ker}\left(P_{-\boldsymbol{\gamma}+\boldsymbol{\nu}}^{*}\right)} \times \widetilde{V}_{\boldsymbol{\gamma}}^{\prime \prime}, \quad([\tau], \sigma) \mapsto\langle\tau, P(\sigma)\rangle,
$$

and that the corresponding map

$$
\widetilde{V}_{\boldsymbol{\gamma}}^{\prime \prime} \rightarrow\left(\frac{\operatorname{Ker}\left(P_{-\boldsymbol{\gamma}-\boldsymbol{\nu}}^{*}\right)}{\operatorname{Ker}\left(P_{-\boldsymbol{\gamma}+\boldsymbol{\nu}}^{*}\right)}\right)^{*}
$$

is injective. This proves that

$$
\operatorname{dim}\left(\widetilde{V}_{\boldsymbol{\gamma}}^{\prime \prime}\right) \leq \operatorname{dim}\left(\operatorname{Ker}\left(P_{-\boldsymbol{\gamma}-\boldsymbol{\nu}}^{*}\right)\right)-\operatorname{dim}\left(\operatorname{Ker}\left(P_{-\boldsymbol{\gamma}+\boldsymbol{\nu}}^{*}\right)\right) .
$$

On the other hand, the change of index formula shows that

$$
\begin{aligned}
\operatorname{dim}\left(\widetilde{V}_{\boldsymbol{\gamma}}^{\prime}\right)+\operatorname{dim}\left(\widetilde{V}_{\boldsymbol{\gamma}}^{\prime \prime}\right)= & \operatorname{dim}\left(\operatorname{Ker}\left(P_{\boldsymbol{\gamma}-\boldsymbol{\nu}}\right)\right)-\operatorname{dim}\left(\operatorname{Ker}\left(P_{-\boldsymbol{\gamma}+\boldsymbol{\nu}}^{*}\right)\right) \\
& -\operatorname{dim}\left(\operatorname{Ker}\left(P_{\boldsymbol{\gamma}+\boldsymbol{\nu}}\right)\right)+\operatorname{dim}\left(\operatorname{Ker}\left(P_{-\boldsymbol{\gamma}-\boldsymbol{\nu}}^{*}\right)\right) .
\end{aligned}
$$

Subtracting Equation (8.6) from (8.7) proves the desired inequality.

\section{Fredholm results for elliptic operators on conifolds}

We now want to see how to achieve analogous results for certain elliptic operators on conifolds. In parallel with Section 7 it is possible to develop an abstract definition and theory of AC operators, analogous to that of asymptotically translation-invariant operators on A.Cyl. manifolds. For simplicity, however, we will limit ourselves to the special case of the Laplace operator acting on functions. This already contains the main ideas of the general theory.

Let $(L, g)$ be a conifold. Consider the weighted spaces introduced in Definition 6.6. As in Section 7 we denote the dual space $\left(W_{k, \boldsymbol{\beta}}^{p}\right)^{*}$ by $W_{-k,-\boldsymbol{\beta}-m}^{p^{\prime}}$. This choice of weights is compatible with the identifications of Remark 6.16, and the properties of these dual spaces are analogous to those seen in Section 7. It follows directly from the definitions that

$$
\nabla: W_{k, \boldsymbol{\beta}}^{p} \rightarrow W_{k-1, \boldsymbol{\beta}-1}^{p}
$$


is a continuous operator. Equation (2.3) then implies that $\Delta_{g}$ extends to a continuous map

$$
\Delta_{\boldsymbol{\beta}}: W_{k, \boldsymbol{\beta}}^{p} \rightarrow W_{k-2, \boldsymbol{\beta}-2}^{p}
$$

The following result is closely related to Lemma 7.3 and uses the fact that $\Delta_{g}$ is formally self-adjoint.

Lemma 9.1. Let $(L, g)$ be a conifold. Choose $u \in W_{k, \boldsymbol{\beta}}^{p}, v \in W_{2-k, 2-\boldsymbol{\beta}-m}^{p^{\prime}}$. Then

$$
\left\langle v, \Delta_{g} u\right\rangle=\langle d v, d u\rangle=\left\langle\Delta_{g} v, u\right\rangle
$$

Proof. Using the appropriate dualities, each expression in Equation (9.2) defines by composition a continuous bilinear map $(u, v) \in W_{k, \beta}^{p} \times$ $W_{2-k, 2-\beta-m}^{p^{\prime}} \rightarrow \mathbb{R}$. Since $\Delta_{g}=d^{*} d$ the equalities hold on the dense subsets $C_{c}^{\infty} \times C_{c}^{\infty}$. By continuity the equalities thus continue to hold on the full Sobolev spaces.

We now want to investigate the Fredholm properties of $\Delta_{\beta}$. It is initially useful to distinguish between the AC and CS case. To begin, let $(L, g)$ be an AC manifold with ends $S_{j}$ and links $\Sigma_{j}$. The starting point for the Fredholm theory is then the following observation.

Lemma 9.2. Let $\left(\Sigma, g^{\prime}\right)$ be a Riemannian manifold. Let the corresponding cone $C:=\Sigma \times(0, \infty)$ have the conical metric $\tilde{g}:=d r^{2}+r^{2} g^{\prime}$. Let $\Delta_{\tilde{g}}$ denote the corresponding Laplace operator on functions. Then, under the substitution $r=\mathrm{e}^{z}$, the operator $r^{2} \Delta_{\tilde{g}}$ coincides with the translation-invariant operator

$$
P_{\infty}:=-(\partial z)^{2}+(2-m) \partial z+\Delta_{\Sigma}
$$

on the cylinder $\Sigma \times \mathbb{R}$.

Proof. Recall that in any local coordinate system the Laplace operator on functions is given by the formula

$$
\Delta_{g}=-\frac{1}{\sqrt{g}} \partial_{j}\left(\sqrt{g} g^{i j} \partial_{i}\right)
$$


Let $U$ be a local chart on $\Sigma$ so that $U \times(0, \infty)$ is a local chart on $C$. Equation (9.4) then shows that

$$
\Delta_{\tilde{g}}=-(\partial r)^{2}-\frac{m-1}{r} \partial r+r^{-2} \Delta_{\Sigma} .
$$

The substitution $r=\mathrm{e}^{z}$ implies $r \partial r=\partial z$. The claim is then a simple calculation.

Lemma 9.2 allows us to study the Fredholm properties of $\Delta_{g}$ by building an equivalent problem for an A.Cyl. manifold, as follows. We use the notation of Section 7.

Multiplication by $\rho^{2}$ defines an isometry $W_{k-2, \boldsymbol{\beta}-2}^{p} \simeq W_{k-2, \boldsymbol{\beta}}^{p}$. Thus $\Delta_{\boldsymbol{\beta}}$ in Equation (9.1) is Fredholm iff the operator

$$
\rho^{2} \Delta_{\boldsymbol{\beta}}: W_{k, \boldsymbol{\beta}}^{p} \rightarrow W_{k-2, \boldsymbol{\beta}}^{p}
$$

is Fredholm. Now consider the A.Cyl. manifold $(L, h)$, where $h=\rho^{-2} g$. It follows from Equation (2.3) and Lemma 9.2 that the operator $P:=\rho^{2} \Delta_{g}$ is asymptotic in the sense of Definition 7.1 to the translation-invariant operator $P_{\infty}$ of Equation (9.3). One can check that the convergence rate $\boldsymbol{\nu}$ of $P$ coincides with the convergence rate $\boldsymbol{\nu}$ of the AC manifold, cf. Definition 6.2.

It is simple to verify that the equation $P_{\infty}\left(\mathrm{e}^{(\gamma+i \delta) z} \sigma(\theta)\right)=0$ is equivalent to the following eigenvalue problem on the link:

$$
\Delta_{\Sigma_{j}} \sigma=\left[(\gamma+i \delta)^{2}+(m-2)(\gamma+i \delta)\right] \sigma .
$$

Using the fact that the eigenvalues $e_{n}^{j}$ of $\Delta_{\Sigma_{j}}$ are real and non-negative, it follows that $\delta=0$ and that $\gamma$ satisfies $\gamma^{2}+(m-2) \gamma=e_{n}^{j}$ for some $n$, i.e.,

$$
\gamma=\frac{(2-m) \pm \sqrt{(2-m)^{2}+4 e_{n}^{j}}}{2} .
$$

This shows that, for this particular operator, $\mathcal{C}_{P_{\infty}}^{j}=\mathcal{D}_{P_{\infty}}^{j}$. It also follows from Lemma 9.2 that the equation $P_{\infty}\left(\mathrm{e}^{\gamma z} \sigma(\theta)\right)=0$ is equivalent to $\Delta_{\tilde{g}}\left(r^{\gamma} \sigma\right)=0$. Thus

$$
V_{\gamma}^{j}=\left\{r^{\gamma} \sigma(\theta): \Delta_{\tilde{g}}\left(r^{\gamma} \sigma\right)=0\right\}
$$

i.e., $V_{\gamma}^{j}$ coincides with the space of homogeneous harmonic functions of degree $\gamma$ on the cone $\Sigma_{j} \times(0, \infty)$. 
Varying the choice of eigenvalue $e_{n}^{j}$ gives the set of exceptional weights for $P_{\infty}$ on the end $S_{j}$. Repeating this for each end defines the set $\mathcal{D}_{P_{\infty}} \subset \mathbb{R}^{e}$. According to Theorem 7.7 these are the weights for which the operator $P$ is not Fredholm with respect to the Sobolev spaces of $(L, h)$. However, recall from Remark 6.16 that the Sobolev spaces of $(L, g)$ and $(L, h)$ coincide. Thus $\mathcal{D}_{P_{\infty}} \subset \mathbb{R}^{e}$ are also the weights for which the operators of Equations (9.6), (9.1) are not Fredholm.

Remark 9.3. Note that in this particular case (and in the analogous case presented in Example 7.12) the generalized eigenvalue problem introduced in Remark 7.6 has reduced to an eigenvalue problem in the usual sense.

It is also fairly straightforward to verify that, for this operator $P_{\infty}$, the spaces $\widetilde{V}_{\gamma+i \delta}^{j}$ and $V_{\gamma+i \delta}^{j}$ coincide, cf. Joyce [5] Proposition 2.4 for details. This allows us to simplify the definition of the multiplicity $m(\gamma)$.

The situation for CS manifolds is similar. The change of variables $r=\mathrm{e}^{-z}$ introduces a change of sign in Equation (9.3). This sign is later cancelled by a change of sign in the identification of Sobolev spaces of $(L, g)$ and $(L, h)$. The final result is thus identical to the AC case. Combining these results leads to the following conclusion.

Corollary 9.4. Let $(L, g)$ be a conifold with e ends. For each end $S_{j}$ with link $\Sigma_{j}$ let $e_{n}^{j}$ denote the eigenvalues of the positive Laplace operator $\Delta_{\Sigma_{j}}$ and define the set of "exceptional weights" $\mathcal{D}^{j}:=\left\{\gamma_{j}\right\} \subseteq \mathbb{R}$ as in Equation (9.8). Given any weight $\gamma \in \mathbb{R}$ define $V_{\gamma}^{j}$ as in Equation (9.9) and let $m^{j}(\gamma)$ denote its dimension. Given any weight $\gamma \in \mathbb{R}^{e}$ set $m(\boldsymbol{\gamma}):=\sum_{j=1}^{e} m^{j}\left(\gamma_{j}\right)$. Let $\mathcal{D} \subseteq \mathbb{R}^{e}$ denote the set of weights $\gamma$ for which $m(\gamma)>0$. Then each multiplicity $m(\gamma)$ is finite and the Laplace operator

$$
\Delta_{g}: W_{k, \boldsymbol{\beta}}^{p} \rightarrow W_{k-2, \boldsymbol{\beta}-2}^{p}
$$

is Fredholm iff $\boldsymbol{\beta} \notin \mathcal{D}$.

The analogue of Theorem 7.9 also holds. For example, assume $L$ is a $C S / A C$ manifold and write $\boldsymbol{\beta}=(\boldsymbol{\mu}, \boldsymbol{\lambda})$. Choose $\left(\boldsymbol{\mu}_{1}, \boldsymbol{\lambda}_{1}\right),\left(\boldsymbol{\mu}_{2}, \boldsymbol{\lambda}_{2}\right) \in \mathbb{R}^{e} \backslash \mathcal{D}$ with $\boldsymbol{\mu}_{1} \geq \boldsymbol{\mu}_{2}, \boldsymbol{\lambda}_{1} \leq \boldsymbol{\lambda}_{2}$. Then

$$
i_{\boldsymbol{\mu}_{2}, \boldsymbol{\lambda}_{2}}\left(\Delta_{g}\right)-i_{\boldsymbol{\mu}_{1}, \boldsymbol{\lambda}_{1}}\left(\Delta_{g}\right)=\sum m(\boldsymbol{\mu}, \boldsymbol{\lambda}),
$$

where the sum is taken over all $(\boldsymbol{\mu}, \boldsymbol{\lambda}) \in \mathcal{D}$ such that $\boldsymbol{\mu}_{1} \geq \boldsymbol{\mu} \geq \boldsymbol{\mu}_{2}, \boldsymbol{\lambda}_{1} \leq$ $\lambda \leq \lambda_{2}$.

In the same way one can also prove the analogue of Proposition 7.11. 


\section{Application: harmonic functions on conifolds}

We can use the results of Sections 8 and 9 to reach a good understanding of the properties of the Laplace operator acting on functions on conifolds. Specifically, we will be interested in the kernel and cokernel of $\Delta_{g}$.

Smooth compact manifolds. Let $(L, g)$ be a smooth compact Riemannian manifold. Let $\Delta_{g}$ denote the positive Laplace operator on functions. Consider the map

$$
\Delta_{g}: W_{k}^{p}(L) \rightarrow W_{k-2}^{p}(L)
$$

For all $p>1$ and $k \in \mathbb{Z}$, standard elliptic regularity shows that any $f \in$ $\operatorname{Ker}\left(\Delta_{g}\right)$ is smooth. The maximum principle then proves that $f$ is constant. Thus $\operatorname{Ker}\left(\Delta_{g}\right)=\mathbb{R}$, independently of the choice of $p, k$.

As seen in Section $2, f \in \operatorname{Im}\left(\Delta_{g}\right)$ iff $\langle u, f\rangle=0$, for all $u \in \operatorname{Ker}\left(\Delta_{g}^{*}\right)$, where $\Delta_{g}^{*}$ is the operator dual to that of Equation (10.1). As in Lemma 7.3 we can identify this with the formal adjoint operator. However, $\Delta_{g}$ is formally self-adjoint, i.e., the operators $\Delta_{g}$ and $\Delta_{g}^{*}$ coincide on smooth functions. By continuity they continue to coincide when extended to any Sobolev space. Thus $\operatorname{Ker}\left(\Delta_{g}^{*}\right)=\operatorname{Ker}\left(\Delta_{g}\right)=\mathbb{R}$. As in Equation (7.3) we find $<u, f>=\int_{L} u f \operatorname{vol}_{g}$. It follows that $\operatorname{Im}\left(\Delta_{g}\right)=\left\{f \in W_{k-2}^{p}(L): \int_{L} f \operatorname{vol}_{g}=\right.$ $0\}$. In particular, $\Delta_{g}$ has index zero.

$A C$ manifolds. Let $(L, g)$ be a AC manifold with convergence rate $\boldsymbol{\nu}<0$ as in Definition 6.2. Let $\Delta_{g}$ denote the positive Laplace operator on weighted Sobolev spaces of functions, as in Equation (9.1). For simplicity, we will restrict our attention to the case of $L$ with two ends.

Each end defines exceptional weights, plotted as points on the horizontal and vertical axes of figure 1. Each exceptional weight gives rise to an exceptional hyperplane, plotted as a vertical or horizontal line. The Laplacian is Fredholm for weights $\boldsymbol{\beta}=\left(\beta_{1}, \beta_{2}\right)$ which are non-exceptional, i.e., which do not lie on these lines. The arrow indicates the direction in which the corresponding Sobolev spaces, thus the kernel of $\Delta_{g}$, become bigger.

Choose $\boldsymbol{\beta}$ non-exceptional. For all $p>1$ and $k \in \mathbb{Z}$, standard elliptic regularity proves that any $f \in \operatorname{Ker}\left(\Delta_{g}\right)$ is smooth. Furthermore, since $\operatorname{Ker}\left(\Delta_{g}\right)$ is independent of $p$ and $k$, the Sobolev Embedding Theorems show that $f$ has growth of the order $O\left(r^{\boldsymbol{\beta}}\right)$. If $\boldsymbol{\beta}<0$ we can thus apply the maximum principle to conclude that $f \equiv 0$. In other words, $\Delta_{g}$ is injective throughout the quadrant defined by the lower shaded region. Since $\Delta_{g}$ is formally self-adjoint, the same holds for $\Delta_{g}^{*}$. Recall from Section 9 how weights on AC manifolds change under duality. We conclude, following Section 2, that 


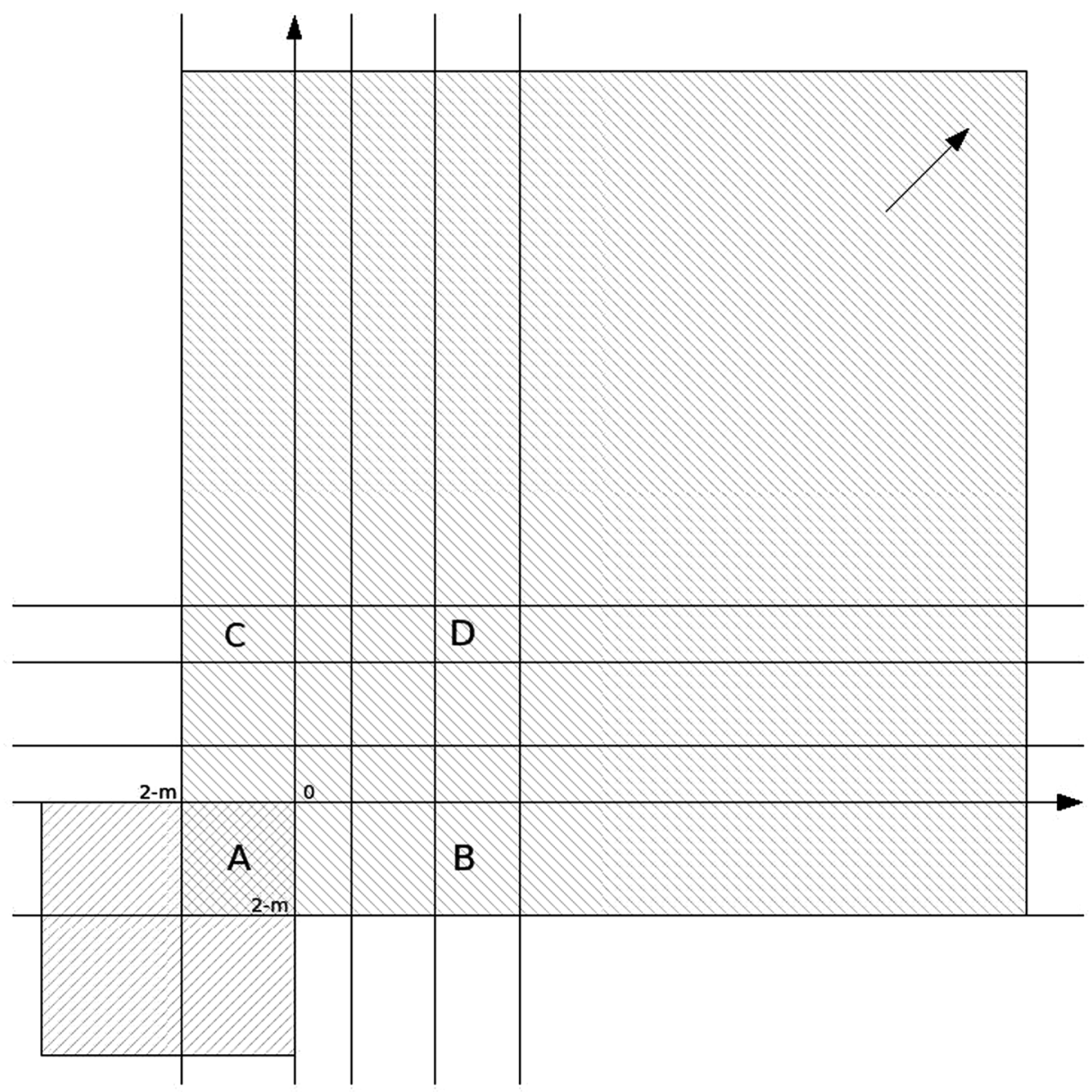

Figure 1: Harmonic functions on AC manifolds.

$\operatorname{Coker}\left(\Delta_{g}\right)=0$ for $\boldsymbol{\beta}>2-m$. In other words, $\Delta_{g}$ is surjective throughout the quadrant defined by the upper shaded region. In particular, the map of Equation (9.1) is an isomorphism and has index zero for $2-m<\boldsymbol{\beta}<0$, i.e., in the region marked by $\mathrm{A}$.

When $\boldsymbol{\beta}>2-m$ the cokernel is independent of the weight. Thus, any change of index corresponds entirely to a change of kernel. Furthermore, $\operatorname{Ker}\left(\Delta_{g}\right)=\operatorname{Ker}\left(\rho^{2} \Delta_{g}\right)$. We can thus use the results of Section 8 to study how the kernel changes as $\boldsymbol{\beta}$ increases. For example, assume we are interested in harmonic functions for some (thus any) $\boldsymbol{\beta}$ in the region $\mathrm{B}$. We can reach this region by keeping $\beta_{2}$ fixed and repeatedly increasing $\beta_{1}$, starting from 
the region A. Each time we cross an exceptional line $x=\gamma$, new harmonic functions on $(L, g)$ are generated by elements $r^{\gamma} \sigma(\theta) \in V_{\gamma}^{1}$. Specifically, these new harmonic functions will be asymptotic to $r^{\gamma} \sigma$ on the first end and to zero on the second end. Using the ideas of Section 8, we can further show that the lower-order terms will have rate $O\left(r^{\gamma+\nu_{1}}\right)$ on the first end and $O\left(r^{\nu_{2}}\right)$ on the second. Analogous results hold for harmonic functions for $\boldsymbol{\beta}$ in the region $\mathrm{C}$. The construction shows that the harmonic functions in the regions $\mathrm{B}$ and $\mathrm{C}$ are linearly independent. We can thus apply the change of index formula to show that harmonic functions in the generic region $\mathrm{D}$ are generated by linear combinations of harmonic functions in the regions B, C.

It may be good to emphasize that the above constructions depend on the specific $(L, g)$ only in terms of the specific exceptional weights, but are otherwise completely independent of $(L, g)$. However, these constructions fail if $\mathrm{D}$ is chosen outside the region where $\Delta_{g}$ is surjective.

CS manifolds. Let $(L, g)$ be a CS manifold with convergence rate $\boldsymbol{\nu}>0$ as in Definition 6.2. As before, let $\Delta_{g}$ denote the positive Laplace operator on weighted Sobolev spaces of functions, as in Equation (9.1). We again restrict our attention to the case of $L$ with two ends.

Figure 2 plots the exceptional weights and lines in this case. Once again the arrow indicates the direction in which the corresponding Sobolev spaces, thus the kernel of $\Delta_{g}$, become bigger. Choose $\boldsymbol{\beta}$ non-exceptional. As before, any $f \in \operatorname{Ker}\left(\Delta_{g}\right)$ is smooth with growth of order $O\left(r^{\boldsymbol{\beta}}\right)$. If $\boldsymbol{\beta}>0$ the maximum principle shows that $f=0$. Now assume $\boldsymbol{\beta}=\frac{2-m}{2}$. In this case $\left(W_{k-2, \boldsymbol{\beta}-2}^{2}\right)^{*}=W_{2-k, \boldsymbol{\beta}}^{2}$. Choose $f \in W_{k, \boldsymbol{\beta}}^{2}$ and assume $\Delta_{g} f=0$. Then, choosing $u=v=f$ in Lemma 9.1 and using regularity, we can conclude $d f=0$ so $f$ is constant. This shows that, for any weight in the region A, $\operatorname{Ker}\left(\Delta_{g}\right)=\mathbb{R}$. As before we also find that, in this region, $\operatorname{Im}\left(\Delta_{g}\right)=\{f \in$ $\left.W_{k-2, \beta-2}^{p}: \int_{L} f \operatorname{vol}_{g}=0\right\}$. In particular, the index of $\Delta_{g}$ is zero.

Now assume $\left(\beta_{1}, \beta_{2}\right)>\left(0, \frac{2-m}{2}\right)$. Then $W_{k, \boldsymbol{\beta}}^{p} \subset W_{k,\left(\frac{2-m}{2}, \frac{2-m}{2}\right)}^{p}$ so our integration by parts argument remains valid. On the other hand, the only constant function in $W_{k, \beta}^{p}$ is zero, so in this case we find that $\Delta_{g}$ is injective. The same holds for $\left(\beta_{1}, \beta_{2}\right)>\left(\frac{2-m}{2}, 0\right)$. Thus $\Delta_{g}$ is injective in the upper shaded region. By duality we deduce that $\Delta_{g}$ is surjective in the lower shaded region.

Now assume $\boldsymbol{\beta}$ crosses from A to B. In this particular case the method used above for AC manifolds fails, because it would require $\Delta_{g}$ to be surjective in the region $\mathrm{A}$. We can however bypass this problem as follows: the change of index formula shows that the index increases by one and we know that the Laplacian is surjective in $\mathrm{B}$, so $\operatorname{Ker}\left(\Delta_{g}\right)=\mathbb{R}$ in $\mathrm{B}$. The same is true for the region $\mathrm{C}$. We can use Section 8 to study the harmonic functions in 


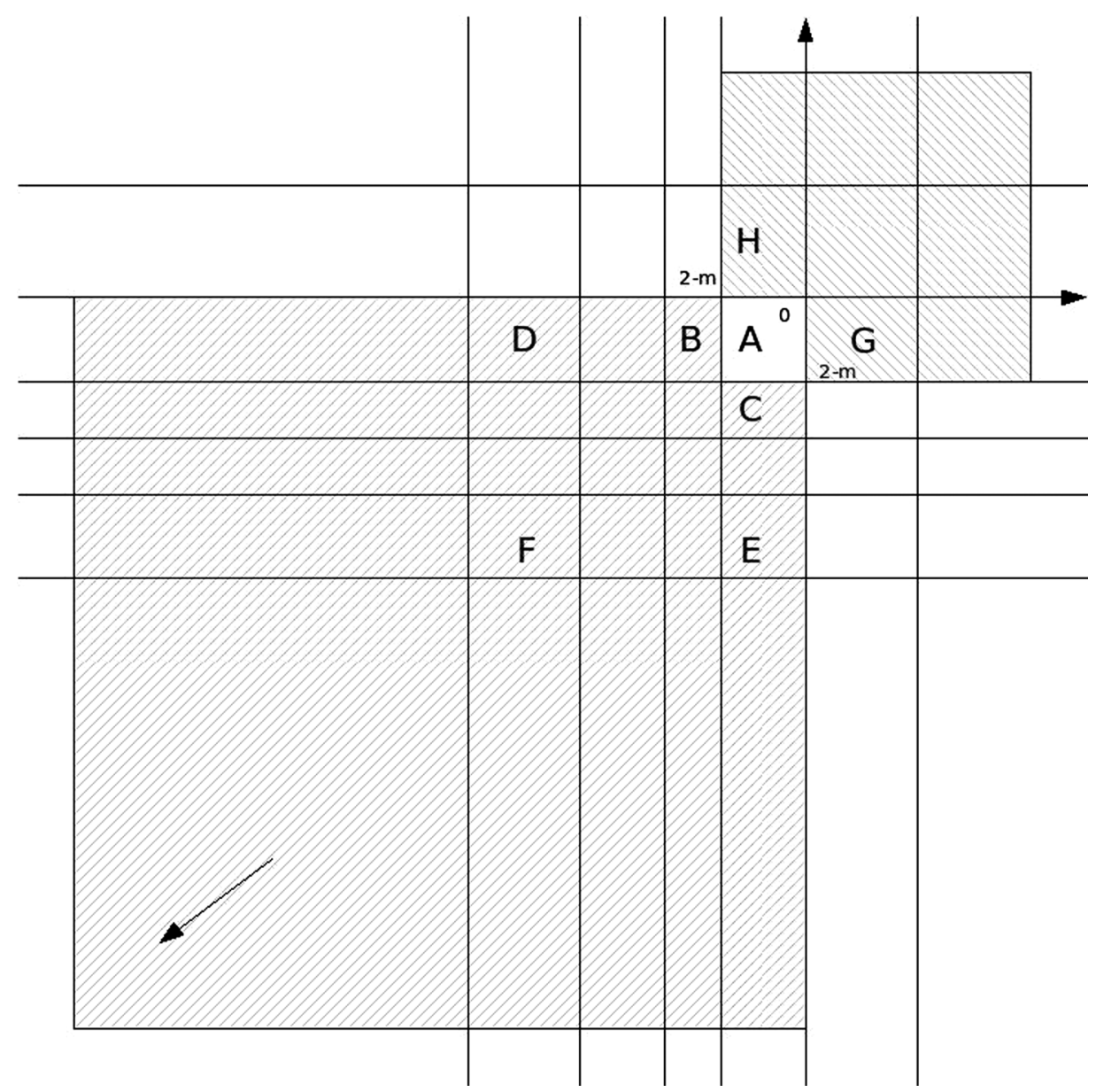

Figure 2: Harmonic functions on CS manifolds.

the lower shaded region. For example, the harmonic functions in $\mathrm{D}$ will be generated by functions which are of the form $r^{\gamma} \sigma+O\left(r^{\gamma+\nu_{1}}\right)$ on the first end and of the form $O\left(r^{\nu_{2}}\right)$ on the second end. Note a difference with respect to AC manifolds: harmonic functions in B and C (more generally, in D and E) are not necessarily linearly independent. Thus we cannot write harmonic functions in $\mathrm{F}$ as the direct sum of harmonic functions in $\mathrm{D}$ and $\mathrm{E}$, as in the $\mathrm{AC}$ case. Once again, harmonic functions elsewhere will be heavily dependent on the specific $(L, g)$.

We may also be interested in the cokernel of $\Delta_{g}$. The change of index formula shows that the dimension of the cokernel increases with $\beta$. For example, the index is -1 in the regions G,H. Since $\Delta_{g}$ is injective here, this 


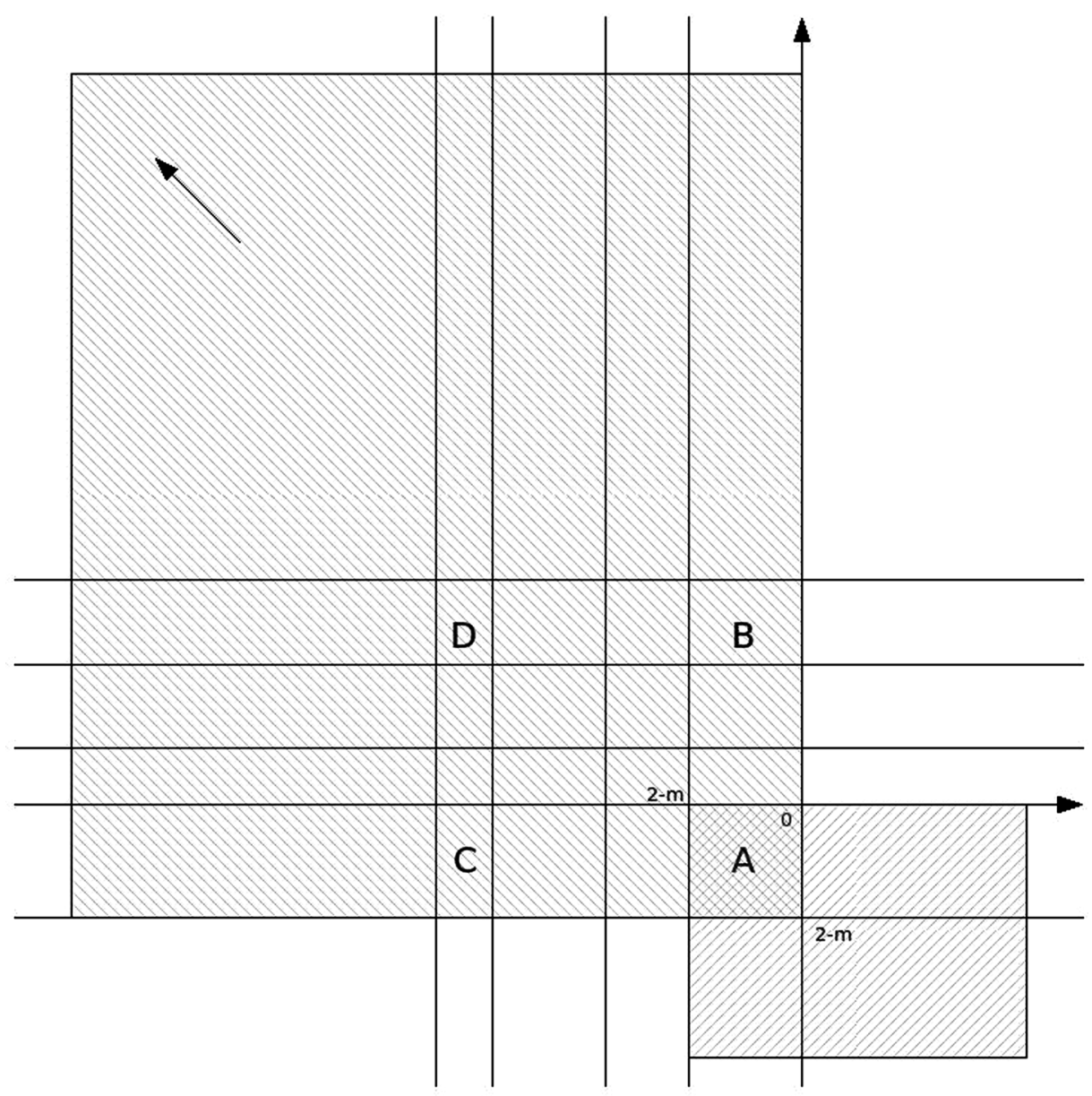

Figure 3: Harmonic functions on CS/AC manifolds.

implies that the cokernel has dimension 1. More generally, the change of index formula allows us to compute the dimension of the cokernel wherever $\Delta_{g}$ is injective. We can also use the ideas of Remark 7.4 to build complements of $\operatorname{Im}\left(\Delta_{g}\right)$ which grow with $\boldsymbol{\beta}$.

$C S / A C$ manifolds. Let $(L, g)$ be a CS/AC manifold with convergence rate $\boldsymbol{\nu}$. Following the same conventions as before, we now turn to figure 3 . Here, the horizontal axis corresponds to the CS end with weight $\mu$ and the vertical axis corresponds to the $\mathrm{AC}$ end with weight $\lambda$.

When $\lambda<0$ and $\mu>2-m$, the maximum principle and integration by parts show that $\Delta_{g}$ is injective. Dually, when $\lambda>2-m$ and $\mu<0, \Delta_{g}$ is surjective. In the region $\mathrm{A}, \Delta_{g}$ is an isomorphism with index zero. Harmonic 
functions in the region B are of the form $r^{\gamma} \sigma+O\left(r^{\gamma+\nu_{2}}\right)$ on the AC end and of the form $O\left(r^{\nu_{1}}\right)$ on the CS end. Harmonic functions in the region $\mathrm{C}$ are of the form $r^{\gamma} \sigma+O\left(r^{\gamma+\nu_{1}}\right)$ on the CS end and of the form $O\left(r^{\nu_{2}}\right)$ on the AC end. Since these functions are linearly independent, their linear combinations give the harmonic functions in the region $\mathrm{D}$.

Example 10.1. $\mathbb{R}^{m}$ with its standard metric can be viewed as a CS/AC manifold, the CS end being a neighbourhood of the origin. In this case all harmonic functions can be written explicitly, so in this case we have exact information on their asymptotics.

\section{Part 3. Conifold connect sums and uniform estimates}

This is the main part of the paper. Our goal is to introduce a certain "parametric connect sum" construction between conifolds; as mentioned in the Introduction, this is the abstract analogue of certain desingularization procedures used in differential geometry, in which an isolated conical singularity is replaced by something smooth or perhaps by a new collection of AC or CS ends. We will show that careful choices of parameters and weights lead to uniform estimates concerning both Sobolev Embedding Theorems and the Laplace operator. These estimates are at the heart of the paper [15]. Readers interested in specific applications of these estimates can thus refer there for details.

\section{Conifold connect sums}

The goal of this section is to define the "parametric connect sum" construction and prove that the scaled and weighted Sobolev constants are independent of the parameter $\boldsymbol{t}$. For simplicity we start with the non-parametric version.

Definition 11.1. Let $(L, g)$ be a conifold, not necessarily connected. Let $S$ denote the union of its ends. A subset $S^{*}$ of $S$ defines a marking on $L$. We can then write $S=S^{*} \amalg S^{* *}$, where $S^{* *}$ is simply the complement of $S^{*}$. We say $S^{*}$ is a $C S$-marking if all ends in $S^{*}$ are CS; it is an $A C$-marking if all ends in $S^{*}$ are AC. We will denote by $d$ the number of ends in $S^{*}$.

If $L$ is weighted via $\boldsymbol{\beta}$ we require that $\beta_{i}=\beta_{j}$, if $S_{i}$ and $S_{j}$ are marked ends belonging to the same connected component of $L$. 
Definition 11.2. Let $\left(L, g, S^{*}\right)$ be a CS-marked conifold. Let $\Sigma^{*}, C^{*}$ denote the links and cones corresponding to $S^{*}$, as in Definition 6.2. Given any end $S_{i} \subseteq S^{*}$ let $\phi_{i}: \Sigma_{i} \times(0, \epsilon] \rightarrow \overline{S_{i}}$ be the diffeomorphism of Definition 6.2.

Let $\left(\hat{L}, \hat{g}, \hat{S}^{*}\right)$ be an AC-marked conifold. Let $\hat{\Sigma}^{*}, \hat{C}^{*}, \hat{\phi}_{i}: \hat{\Sigma}_{i} \times[\hat{R}, \infty) \rightarrow$ $\overline{\hat{S}_{i}}$ denote the corresponding links, cones and diffeomorphisms, as above.

We say that $L$ and $\hat{L}$ are compatible if they satisfy the following assumptions:

1) $C^{*}=\hat{C}^{*}$. Up to relabelling the ends, we may assume that $C_{i}^{*}=\hat{C}_{i}^{*}$.

2) $\hat{R}<\epsilon$. We can then identify appropriate subsets of $S^{*}$ and $\hat{S}^{*}$ via the maps $\hat{\phi}_{i} \circ \phi_{i}^{-1}$.

3) On each marked AC end, the metrics $\hat{\phi}_{i}^{*} \hat{g}$ and $\tilde{g}_{i}$ are scaled-equivalent in the sense of Definition 4.3. Analogously, on each marked CS end, the metrics $\phi_{i}^{*} g$ and $\tilde{g}_{i}$ are scaled-equivalent in the sense of Definition 4.3.

If $L$ is weighted via $\boldsymbol{\beta}$ and $\hat{L}$ is weighted via $\hat{\boldsymbol{\beta}}$ we further require that, on the marked ends, the corresponding constants satisfy $\beta_{i}=\hat{\beta}_{i}$ and that $\hat{\beta}_{i}=\hat{\beta}_{j}$ if $\hat{S}_{i}$ and $\hat{S}_{j}$ are marked ends in the same connected component of $\hat{L}$.

Remark 11.3. The condition $\hat{R}<\epsilon$ may seem rather strong. However, let $\left(L, g, S^{*}\right)$ be CS-marked, $\left(\hat{L}, \hat{g}, \hat{S}^{*}\right)$ be AC-marked and $C^{*}=\hat{C}^{*}$. As seen in Remark 6.3 , by making $\hat{R}$ larger if necessary it is possible to assume that the metrics $\hat{\phi}_{i}^{*} \hat{g}, \tilde{g}_{i}$ on $\Sigma_{i} \times[\hat{R}, \infty)$ are scaled-equivalent in the sense of Definition 4.3. Lemma 6.10 then shows that the metrics $\hat{\phi}_{t, i}^{*}\left(t^{2} \hat{g}\right), \tilde{g}_{i}$ on $\Sigma_{i} \times[t \hat{R}, \infty)$ are also scaled-equivalent, with the same bounds. Analogously, by making $\epsilon$ smaller if necessary, we can assume that the metrics $\phi_{i}^{*} g, \tilde{g}_{i}$ on $\Sigma_{i} \times(0, \epsilon]$ are scaled-equivalent. By first making $\hat{R}$ large and $\epsilon$ small and then rescaling to satisfy the condition $\hat{R}<\epsilon$ we thus obtain compatible conifolds in the sense of Definition 11.2.

Definition 11.4. Let $\left(L, g, S^{*}\right),\left(\hat{L}, \hat{g}, \hat{S}^{*}\right)$ be compatible marked conifolds. We define the connect sum of $L$ and $\hat{L}$ as follows. We set

$$
\hat{L} \# L:=\left(\hat{L} \backslash \hat{S}^{*}\right) \cup\left(\Sigma^{*} \times[\hat{R}, \epsilon]\right) \cup\left(L \backslash S^{*}\right),
$$

where the boundary of $\hat{L} \backslash \hat{S}^{*}$ is identified with $\Sigma^{*} \times\{\hat{R}\}$ via the maps $\hat{\phi}_{i}$ and the boundary of $L \backslash S^{*}$ is identified with $\Sigma^{*} \times\{\epsilon\}$ via the maps $\phi_{i}$. We can endow this manifold with any metric $\hat{g} \# g$ which restricts to $\hat{g}$ on $\hat{L} \backslash \hat{S}^{*}$ 
and to $g$ on $L \backslash S^{*}$. Then $\hat{L} \# L$ is a conifold. Its ends are $\hat{S}^{* *} \amalg S^{* *}$. We call $\Sigma^{*} \times[\hat{R}, \epsilon]$ the neck region of $\hat{L} \# L$.

Given radius functions $\rho$ on $L$ and $\hat{\rho}$ on $\hat{L}$ we can endow $\hat{L} \# L$ with the radius function

$$
\hat{\rho} \# \rho:= \begin{cases}\hat{\rho} & \text { on } \hat{L} \backslash \hat{S}^{*} \\ r & \text { on } \Sigma^{*} \times[\hat{R}, \epsilon] \\ \rho & \text { on } L \backslash S^{*}\end{cases}
$$

If $L, \hat{L}$ are weighted via $\boldsymbol{\beta}, \hat{\boldsymbol{\beta}}$ then $\hat{L} \# L$ is weighted via the function

$$
\hat{\boldsymbol{\beta}} \# \boldsymbol{\beta}:= \begin{cases}\hat{\boldsymbol{\beta}} & \text { on } \hat{L} \backslash \hat{S}^{*} \\ \boldsymbol{\beta}_{\mid S^{*}} & \text { on } \Sigma^{*} \times[\hat{R}, \epsilon] \\ \boldsymbol{\beta} & \text { on } L \backslash S^{*}\end{cases}
$$

Example 11.5. Let $\bar{L}$ be a smooth $m$-dimensional submanifold of $\mathbb{R}^{n}$, endowed with the induced metric. Assume that it is either compact or that it has AC ends: e.g., it could be a collection of $m$-planes in $\mathbb{R}^{n}$. Now assume it has transverse self-intersection points $x_{1}, \ldots, x_{k} \in \mathbb{R}^{n}$. For each $x_{i}$ choose a ball $B\left(x_{i}, \epsilon\right)$ in $\mathbb{R}^{n}$. Then $L:=\bar{L} \backslash\left\{x_{1}, \ldots, x_{k}\right\}$ is a conifold with $s$ CS ends defined by the connected components of $\left(B\left(x_{1}, \epsilon\right) \cup \cdots \cup B\left(x_{k}, \epsilon\right)\right) \cap L$. The corresponding cones are copies of $\mathbb{R}^{m}$. Choose a pair $S_{1}, S_{2}$ of connected components of $B\left(x_{1}, \epsilon\right) \cap L$ and an appropriately rescaled $m$-dimensional hyperboloid $\hat{L} \subseteq \mathbb{R}^{n}$ asymptotic to the corresponding cones $C_{1}, C_{2}$. Then $L$, $\hat{L}$ are compatible and $\hat{L} \# L$ is an abstract Riemannian manifold, which we can think of as a desingularization of $\bar{L}$. Our hypothesis in Definition 11.1 that $L, \hat{L}$ are not necessarily connected allows us to extend this construction to intersection points of distinct submanifolds and to desingularize all points simultaneously.

Since $\hat{L} \# L$ is again a conifold it is clear that all versions of the Sobolev Embedding Theorems continue to hold for it. Note that $\hat{S}^{* *} \cup S^{* *}$ might also be empty: in this case $\hat{L} \# L$ is a smooth compact manifold. We now consider the parametric version of this construction.

Definition 11.6. Let $\left(L, g, S^{*}\right),\left(\hat{L}, \hat{g}, \hat{S}^{*}\right)$ be compatible marked conifolds with $d$ marked ends. Let $(\rho, \boldsymbol{\beta})$, respectively, $(\hat{\rho}, \hat{\boldsymbol{\beta}})$, be corresponding radius functions and weights. Choose parameters $\boldsymbol{t}=\left(t_{1}, \ldots, t_{d}\right)>0$ sufficiently small. We assume that $\boldsymbol{t}$ is compatible with the decomposition of $\hat{L}$ into its connected components: specifically, that $t_{i}=t_{j}$ if $\hat{S}_{i}$ and $\hat{S}_{j}$ belong to the 
same connected component of $\hat{L}$. We then define the parametric connect sum of $L$ and $\hat{L}$ as follows. We set

$$
L_{\boldsymbol{t}}:=\left(\hat{L} \backslash \hat{S}^{*}\right) \cup\left(\cup_{\Sigma_{i} \subseteq \Sigma^{*}} \Sigma_{i} \times\left[t_{i} \hat{R}, \epsilon\right]\right) \cup\left(L \backslash S^{*}\right),
$$

where the components of the boundary of $\hat{L} \backslash \hat{S}^{*}$ are identified with the $\Sigma_{i} \times\left\{t_{i} \hat{R}\right\}$ via maps $\hat{\phi}_{t_{i}, i}$ defined as in Lemma 6.10 and the components of the boundary of $L \backslash S^{*}$ are identified with the $\Sigma_{i} \times\{\epsilon\}$ via the maps $\phi_{i}$. Choose $\tau \in(0,1)$. If the $t_{i}$ are sufficiently small, we find $t_{i} \hat{R}<t_{i}^{\tau}<2 t_{i}^{\tau}<\epsilon$. Choose any metric $g_{\boldsymbol{t}}$ on $L_{\boldsymbol{t}}$ such that, for each $\Sigma_{i} \subseteq \Sigma^{*}$,

$$
g_{\boldsymbol{t}}:= \begin{cases}t_{i}^{2} \hat{g} & \text { on the corresponding component of } \hat{L} \backslash \hat{S}^{*} \\ \hat{\phi}_{t_{i}, i}^{*}\left(t_{i}^{2} \hat{g}\right) & \text { on } \Sigma_{i} \times\left[t_{i} \hat{R}, t_{i}^{\tau}\right] \\ \phi_{i}^{*} g & \text { on } \Sigma_{i} \times\left[2 t_{i}^{\tau}, \epsilon\right] \\ g & \text { on } L \backslash S^{*}\end{cases}
$$

and such that, for all $j \geq 0$ and as $t \rightarrow 0$,

$$
\sup _{\Sigma_{i} \times\left[t_{i}^{\tau}, 2 t_{i}^{\tau}\right]}\left|\widetilde{\nabla}^{j}\left(g_{\boldsymbol{t}}-\tilde{g}_{i}\right)\right|_{r^{-2} \tilde{g}_{i} \otimes \tilde{g}_{i}} \rightarrow 0 .
$$

We endow $L_{\boldsymbol{t}}$ with the radius function

$$
\rho_{\boldsymbol{t}}:= \begin{cases}t_{i} \hat{\rho} & \text { on the corresponding component of } \hat{L} \backslash \hat{S}^{*} \\ r & \text { on } \Sigma_{i} \times\left[t_{i} \hat{R}, \epsilon\right] \\ \rho & \text { on } L \backslash S^{*}\end{cases}
$$

and the weight

$$
\boldsymbol{\beta}_{\boldsymbol{t}}:= \begin{cases}\hat{\boldsymbol{\beta}} & \text { on } \hat{L} \backslash \hat{S}^{*} \\ \beta_{i} & \text { on } \Sigma_{i} \times\left[t_{i} \hat{R}, \epsilon\right] \\ \boldsymbol{\beta} & \text { on } L \backslash S^{*}\end{cases}
$$

We now need to define the weight function $w_{\boldsymbol{t}}$. As in Corollary 6.11 , the simplest case is when $\hat{\boldsymbol{\beta}}$ is constant on each connected component of $\hat{L}$. We then define

$$
w_{\boldsymbol{t}}:=\rho_{t}^{-\boldsymbol{\beta}_{t}}= \begin{cases}\left(t_{i} \hat{\rho}\right)^{-\hat{\beta}_{i}} & \text { on the corresponding component of } \hat{L} \backslash \hat{S}^{*} \\ r^{-\beta_{i}} & \text { on } \Sigma_{i} \times\left[t_{i} \hat{R}, \epsilon\right] \\ \rho^{-\boldsymbol{\beta}} & \text { on } L \backslash S^{*}\end{cases}
$$


For general weights $\hat{\boldsymbol{\beta}}$, we need to modify the weight function. As in Corollary 6.11 , on the $i$ th component of $\hat{L}$ consider the constant "reference" weight $\hat{\beta}_{i}$. We then define

$$
w_{\boldsymbol{t}}:= \begin{cases}\left(t_{i}^{\frac{\hat{\beta}_{i}-\hat{\beta}}{\hat{\beta}}} t_{i} \hat{\rho}\right)^{-\hat{\boldsymbol{\beta}}} & \text { on the corresponding component of } \hat{L} \backslash \hat{S}^{*} \\ r^{-\beta_{i}} & \text { on } \Sigma_{i} \times\left[t_{i} \hat{R}, \epsilon\right] \\ \rho^{-\boldsymbol{\beta}} & \text { on } L \backslash S^{*}\end{cases}
$$

We may equivalently write this as

$$
w_{\boldsymbol{t}}:= \begin{cases}t_{i}^{\hat{\boldsymbol{\beta}}-\hat{\beta}_{i}} \rho_{\boldsymbol{t}}^{-\boldsymbol{\beta}_{t}}, & \text { on } \hat{L} \backslash \hat{S}^{*} \\ \rho_{\boldsymbol{t}}^{-\boldsymbol{\beta}_{t}}, & \text { elsewhere. }\end{cases}
$$

Using this data, we now define weighted Sobolev spaces $W_{k, \boldsymbol{\beta}_{\boldsymbol{t}}}^{p}$ on $L_{\boldsymbol{t}}$ as in Section 5 . We call $\Sigma_{i} \times\left[t_{i} \hat{R}, \epsilon\right]$ the neck regions of $L_{\boldsymbol{t}}$.

Theorem 11.7. Let $\left(L, g, S^{*}\right),\left(\hat{L}, \hat{g}, \hat{S}^{*}\right)$ be compatible weighted marked conifolds. Define $L_{\boldsymbol{t}}, g_{\boldsymbol{t}}, \rho_{\boldsymbol{t}}$ and $\boldsymbol{\beta}_{\boldsymbol{t}}$ as in Definition 11.6. Then all forms of the weighted Sobolev Embedding Theorems hold uniformly in $\boldsymbol{t}$, i.e., the corresponding Sobolev constants are independent of $\boldsymbol{t}$.

Proof. The proof is similar to that of Corollary 6.8. Let us for the moment pretend that the metrics $g, \hat{g}$ are exactly conical on all ends of $L, \hat{L}$. This allows us to assume that the metrics $g_{\boldsymbol{t}}$ are exactly conical on all ends and neck regions of $L_{\boldsymbol{t}}$ so the assumptions of Theorem 5.1 are satisfied in these regions. On $\hat{L} \backslash \hat{S}^{*}$ we are using rescaled metrics, radius functions and weights as in Corollary 6.11. As seen in Remark 5.2, the assumptions of Theorem 5.1 are $\boldsymbol{t}$-independent, so they are verified here. These assumptions are also verified on $L \backslash S^{*}$ and on the neck regions. We conclude that all forms of the weighted Sobolev Embedding Theorems hold for these metrics, with $\boldsymbol{t}$-independent Sobolev constants.

Let us now go back to the metric $g_{\boldsymbol{t}}$. Recall from Lemma 6.10 that we can assume that, on each end of $L_{\boldsymbol{t}}, g_{\boldsymbol{t}}$ is a $\boldsymbol{t}$-uniformly small perturbation of the conical metric. The same is true also on the neck regions. Specifically, on $\Sigma_{i} \times\left[t_{i} \hat{R}, t_{i}^{\tau}\right]$ Lemma 6.10 shows that

$$
\sup \left|\phi_{t, i}^{*}\left(t_{i}^{2} \hat{g}\right)-\tilde{g}_{i}\right| \leq C_{0} \hat{R}^{\hat{\nu}_{i}}
$$

On $\Sigma_{i} \times\left[t_{i}^{\tau}, 2 t_{i}^{\tau}\right]$ our hypotheses imply

$$
\sup \left|g_{\boldsymbol{t}}-\tilde{g}_{i}\right|_{r^{-2}} \tilde{g}_{i} \otimes \tilde{g}_{i} \leq C_{0}
$$


The analogue is true also on $\Sigma_{i} \times\left[2 t_{i}^{\tau}, \epsilon\right]$, using the estimates provided by Definition 6.2.

These perturbations are all $\boldsymbol{t}$-independent, so according to Theorem 5.1 the weighted Sobolev Embedding Theorems hold also for $g_{\boldsymbol{t}}$, with $\boldsymbol{t}$ independent Sobolev constants.

Remark 11.8. Note that Theorem 11.7 actually requires only $\boldsymbol{t}$-uniform $C^{0}$-bounds over the metrics $g_{t}$. In Definition 11.6, we include control over the higher derivatives and the assumption that the quantities in question tend to zero for use in later sections. The same is also true for various other results, e.g., Corollary 6.8.

We conclude with the following result, which serves to highlight certain properties of $g_{\boldsymbol{t}}$ as $\boldsymbol{t} \rightarrow 0$. This is important for Section 12 .

Lemma 11.9. Consider $g_{\boldsymbol{t}}$ as in Definition 11.6. Choose a neck region in $L_{\boldsymbol{t}}$ and $b \in(0, \tau)$ so that $t_{i} \hat{R}<t_{i}^{\tau}<2 t_{i}^{\tau}<t_{i}^{b}<\epsilon$. Then, on $\Sigma_{i} \times\left[t_{i} \hat{R}, t_{i}^{b}\right]$, the metric $g_{\boldsymbol{t}}$ converges to the rescaled metric $t_{i}^{2} \hat{\phi}_{t, i}^{*} \hat{g}$ in the following sense: for all $j \geq 0$ and as $t \rightarrow 0$,

$$
\sup \left|r^{j} \hat{\nabla}^{j}\left(g_{t}-t_{i}^{2} \hat{\phi}_{t, i}^{*} \hat{g}\right)\right|_{t_{i}^{2} \hat{\phi}_{t, i}^{*} \hat{g} \otimes t_{i}^{2} \hat{\phi}_{t, i}^{*} \hat{g}} \rightarrow 0,
$$

where $\hat{\nabla}$ denotes the Levi-Civita connection defined by $\hat{\phi}_{t_{i}, i}^{*} \hat{g}$ on $\Sigma_{i} \times\left[t_{i} \hat{R}, t_{i}^{b}\right]$.

Proof. Consider the map

$$
\delta_{t_{i}}: \Sigma_{i} \times\left[\hat{R}, t_{i}^{b-1}\right] \rightarrow \Sigma_{i} \times\left[t_{i} \hat{R}, t_{i}^{b}\right], \quad(\theta, r) \mapsto\left(\theta, t_{i} r\right) .
$$

We can use this map to pull the estimate back to $\Sigma_{i} \times\left[\hat{R}, t_{i}^{b-1}\right]$. We can then write it as follows: for all $j \geq 0$ and as $t \rightarrow 0$,

$$
\sup \left|\hat{\nabla}^{j}\left(\delta_{t_{i}}^{*}\left(t_{i}^{-2} g_{\boldsymbol{t}}\right)-\hat{\phi}_{i}^{*} \hat{g}\right)\right|_{r^{-2} \hat{\phi}_{i}^{*} \hat{g} \otimes \hat{\phi}_{i}^{*} \hat{g}} \rightarrow 0
$$

where $\hat{\nabla}$ denotes the Levi-Civita connection defined by $\hat{\phi}_{i}^{*} \hat{g}$ on $\Sigma_{i} \times\left[\hat{R}, t^{b-1}\right]$.

We choose to prove this form of the estimate.

On $\Sigma_{i} \times\left[\hat{R}, t_{i}^{\tau-1}\right]$ it follows from Definition 11.6 that $\delta_{t_{i}}^{*}\left(t_{i}^{-2} g_{\boldsymbol{t}}\right)=\hat{\phi}_{i}^{*} \hat{g}$, so the equation is trivially true. 
On $\Sigma_{i} \times\left[t_{i}^{\tau-1}, 2 t_{i}^{\tau-1}\right]$

$$
\begin{aligned}
& \left|\widetilde{\nabla}^{j}\left(\delta_{t_{i}}^{*}\left(t_{i}^{-2} g_{\boldsymbol{t}}\right)-\tilde{g}_{i}\right)\right|_{r^{-2} \tilde{g}_{i} \otimes \tilde{g}_{i}}=\mid \widetilde{\nabla}^{j}\left(\delta_{t_{i}}^{*}\left(t_{i}^{-2} g_{\boldsymbol{t}}\right)\right. \\
& \left.\left.-\delta_{t_{i}}^{*}\left(t_{i}^{-2} \tilde{g}_{i}\right)\right)\left.\right|_{\delta_{t_{i}}^{*}\left(r / t_{i}\right)^{-2} \delta_{t_{i}}^{*}\left(t_{i}-2\right.} \tilde{g}_{i}\right) \otimes \delta_{t_{i}}^{*}\left(t_{i}^{-2} \tilde{g}_{i}\right) \\
& =\delta_{t_{i}}^{*}\left(\left|\widetilde{\nabla}^{j}\left(t_{i}^{-2} g_{\boldsymbol{t}}-t_{i}^{-2} \tilde{g}_{i}\right)\right|_{\left(r / t_{i}\right)^{-2} t_{i}^{-2} \tilde{g}_{i} \otimes t_{i}^{-2} \tilde{g}_{i}}\right) \\
& =\delta_{t_{i}}^{*}\left(\left|\widetilde{\nabla}^{j}\left(g_{\boldsymbol{t}}-\tilde{g}_{i}\right)\right|_{r^{-2}} \tilde{g}_{i} \otimes \tilde{g}_{i}\right) \rightarrow 0,
\end{aligned}
$$

where the last statement follows from Definition 11.6. Furthermore, it follows from Definition 6.2 that

$$
\left|\widetilde{\nabla}^{j}\left(\hat{\phi}_{i}^{*} \hat{g}-\tilde{g}_{i}\right)\right|_{r^{-2}} \tilde{g}_{i} \otimes \tilde{g}_{i} \leq C_{j} t_{i}^{(\tau-1) \hat{\nu}_{i}} \rightarrow 0
$$

using $(\tau-1) \hat{\nu}_{i}>0$. We have thus found that both metrics of interest converge to the same metric $\tilde{g}_{i}$. The conclusion is a simple computation.

On $\Sigma_{i} \times\left[2 t_{i}^{\tau-1}, t_{i}^{b-1}\right]$, as above and using $g_{\boldsymbol{t}}=\phi_{i}^{*} g$,

$$
\left|\widetilde{\nabla}^{j}\left(\delta_{t_{i}}^{*}\left(t_{i}^{-2} g_{\boldsymbol{t}}\right)-\tilde{g}_{i}\right)\right|_{r^{-2} \tilde{g}_{i} \otimes \tilde{g}_{i}}=\delta_{t_{i}}^{*}\left(\left|\widetilde{\nabla}^{j}\left(\phi_{i}^{*} g-\tilde{g}_{i}\right)\right|_{r^{-2} \tilde{g}_{i} \otimes \tilde{g}_{i}}\right) \leq C_{j} t_{i}^{b \nu_{i}} \rightarrow 0
$$

using $b \nu_{i}>0$. Furthermore,

$$
\left|\widetilde{\nabla}^{j}\left(\hat{\phi}_{i}^{*} \hat{g}-\tilde{g}_{i}\right)\right|_{r^{-2} \tilde{g}_{i} \otimes \tilde{g}_{i}} \leq C_{j}\left(2 t_{i}^{\tau-1}\right)^{\hat{\nu}_{i}} \rightarrow 0 .
$$

Again, combining these estimates implies the claim.

\section{The Laplacian on conifold connect sums}

Let $\left(L, g, \rho, S^{*}\right),\left(\hat{L}, \hat{g}, \hat{\rho}, \hat{S}^{*}\right)$ be compatible marked conifolds. As seen in Section 11, we can define their connect sum $(\hat{L} \# L, \hat{g} \# g, \hat{\rho} \# \rho)$. This is a new conifold so we can study the properties of its Laplace operator as in Section 10.

We start with the case in which $\hat{S}^{* *} \cup S^{* *} \neq \emptyset$, i.e., the set of ends is non-empty. This case actually turns out to be easier than the alternative situation, where $\hat{L} \# L$ is smooth and compact, because we can use weights to force injectivity of the Laplacian.

Non-compact conifolds. Assume the set $\hat{S}^{* *} \cup S^{* *}$ of ends of $\hat{L} \# L$ is nonempty. If weights $\boldsymbol{\beta}, \hat{\boldsymbol{\beta}}$ are non-exceptional for $\Delta_{g}, \Delta_{\hat{g}}$ then the weight $\hat{\boldsymbol{\beta}} \# \boldsymbol{\beta}$ 
is non-exceptional for $\Delta_{\hat{g} \# g}$ so

$$
\Delta_{\hat{g} \# g}: W_{k, \hat{\boldsymbol{\beta}} \# \boldsymbol{\beta}}^{p} \rightarrow W_{k-2, \hat{\boldsymbol{\beta}} \# \boldsymbol{\beta}-2}^{p}
$$

is Fredholm. The same holds for the parametric connect sums $\left(L_{\boldsymbol{t}}, g_{\boldsymbol{t}}, \rho_{\boldsymbol{t}}, \boldsymbol{\beta}_{\boldsymbol{t}}\right)$.

We want to study the invertibility of the Laplace operator. The following result is obvious.

Lemma 12.1. Let $\left(\hat{L}, \hat{g}, \hat{\rho}, \hat{\boldsymbol{\beta}}, \hat{S}^{*}\right)$ be a weighted AC-marked conifold. Assume $\hat{\boldsymbol{\beta}}$ satisfies the conditions

$$
\left\{\begin{array}{l}
\hat{\beta}_{i}<0, \text { for all } A C \text { ends } \hat{S}_{i} \in \hat{S} \\
\hat{\beta}_{i}>2-m, \text { for all } C S \text { ends } \hat{S}_{i} \in \hat{S}
\end{array}\right.
$$

so that $\Delta_{\hat{g}}$ is injective.

Let $\left(L, g, \rho, \boldsymbol{\beta}, S^{*}\right)$ be a weighted CS-marked conifold. Assume $\boldsymbol{\beta}$ satisfies the conditions

$$
\left\{\begin{array}{l}
\beta_{i}<0, \text { for all } A C \text { ends } S_{i} \in S \\
\beta_{i}>2-m, \text { for all } C S \text { ends } S_{i} \in S
\end{array}\right.
$$

This is not yet sufficient to conclude that $\Delta_{g}$ is injective because the set of $A C$ ends might be empty. To obtain injectivity we must furthermore assume that each connected component of $L$ has at least one end, e.g., $S^{\prime}$, satisfying the condition

$$
\left\{\begin{array}{l}
\beta^{\prime}<0, \text { if } S^{\prime} \text { is } A C \\
\beta^{\prime}>0, \text { if } S^{\prime} \text { is } C S
\end{array}\right.
$$

Now assume that $L, \hat{L}$ are compatible. Then, for all ends $S_{i} \in S^{*}$, $2-m<\beta_{i}<0$. This implies that $S^{\prime} \in S^{* *}$ so $\hat{L} \# L$ has at least one end. Furthermore, $\hat{\boldsymbol{\beta}} \# \boldsymbol{\beta}$ satisfies the conditions

$$
\left\{\begin{array}{l}
\hat{\boldsymbol{\beta}} \# \boldsymbol{\beta}_{\mid S_{i}}<0, \text { for all } A C \text { ends } S_{i} \in \hat{S}^{* *} \cup S^{* *} \\
\hat{\boldsymbol{\beta}} \# \boldsymbol{\beta}_{\mid S_{i}}>2-m, \text { for all } C S \text { ends } S_{i} \in \hat{S}^{* *} \cup S^{* *}
\end{array}\right.
$$

Together with the condition on $S^{\prime}$, this implies that $\Delta_{\hat{g} \# g}$ is injective. 
If furthermore $\boldsymbol{\beta}, \hat{\boldsymbol{\beta}}$ are non-exceptional for $\Delta_{g}, \Delta_{\hat{g}}$ then

$$
\Delta_{\hat{g} \# g}: W_{k, \hat{\boldsymbol{\beta}} \# \boldsymbol{\beta}}^{p} \rightarrow W_{k-2, \hat{\boldsymbol{\beta}} \# \boldsymbol{\beta}-2}^{p}
$$

is a topological isomorphism onto its image so there exists $C>0$ such that, for all $f \in W_{k, \hat{\boldsymbol{\beta}} \# \boldsymbol{\beta}}^{p}$

$$
\|f\|_{W_{k, \hat{\boldsymbol{\beta}} \# \boldsymbol{\beta}}^{p}} \leq C\|\Delta f\|_{W_{k-2, \hat{\boldsymbol{\beta}} \# \boldsymbol{\beta}-2}^{p}} .
$$

For the constant $C$ in Lemma 12.1 one can choose the norm of the inverse map $\left(\Delta_{\hat{g} \# g}\right)^{-1}$, as in Equation (2.4). The analogous result holds also for parametric connect sums. We now want to show that, in this case, the invertibility constant $C$ can be chosen to be $\boldsymbol{t}$-independent. In other words, there exists a $\boldsymbol{t}$-uniform upper bound on the norms of the inverse maps $\left(\Delta_{g_{t}}\right)^{-1}$.

Theorem 12.2. Let $\left(L, g, \rho, \boldsymbol{\beta}, S^{*}\right),\left(\hat{L}, \hat{g}, \hat{\rho}, \hat{\boldsymbol{\beta}}, \hat{S}^{*}\right)$ be marked compatible conifolds satisfying all the conditions of Lemma 12.1. Define $\left(L_{\boldsymbol{t}}, g_{\boldsymbol{t}}, \rho_{\boldsymbol{t}}, \boldsymbol{\beta}_{\boldsymbol{t}}\right)$ as in Definition 11.6. Then there exists $C>0$ such that, for all $f \in$ $W_{k, \boldsymbol{\beta}_{t}}^{p}\left(L_{\boldsymbol{t}}\right)$,

$$
\|f\|_{W_{k, \beta_{t}}^{p}} \leq C\left\|\Delta_{g_{t}} f\right\|_{W_{k-2, \beta_{t}-2}^{p}} .
$$

Proof. To simplify the notation let us assume that all $t_{i}$ coincide: we can then work with a unique parameter $t$. The general case is analogous.

Let $C_{g}$ denote an invertibility constant for $\Delta_{g}$ on $L$, i.e., for all $f \in$ $W_{k, \boldsymbol{\beta}}^{p}(L)$,

$$
\|f\|_{W_{k, \boldsymbol{\beta}}^{p}} \leq C_{g}\left\|\Delta_{g} f\right\|_{W_{k-2, \boldsymbol{\beta}-2}^{p}} .
$$

Let $C_{\hat{g}}$ denote an analogous constant for $\Delta_{\hat{g}}$ on $\hat{L}$.

Choose constants $a, b$ satisfying $0<b<a<\tau$ and a smooth decreasing function $\eta: \mathbb{R} \rightarrow[0,1]$ such that $\eta(s)=1$ for $s \leq b$ and $\eta(s)=0$ for $s \geq a$. Then the function $\eta_{t}(r):=\eta(\log r / \log t):(0, \infty) \rightarrow[0,1]$ has the following properties:

(1) $\eta_{t}$ is smooth increasing, $\eta_{t}(r)=0$ for $r \leq t^{a}, \eta_{t}(r)=1$ for $r \geq t^{b}$.

(2) For all $k \geq 1$ there exists $C_{k}>0$ such that

$$
\left|r^{k} \frac{\partial^{k} \eta_{t}}{(\partial r)^{k}}(r)\right| \leq \frac{C_{k}}{|\log t|} \rightarrow 0 \quad \text { as } t \rightarrow 0
$$

We set $\eta_{t}^{\prime}(r):=\frac{\partial \eta_{t}}{\partial r}(r), \eta_{t}^{\prime \prime}(r):=\frac{\partial^{2} \eta_{t}}{(\partial r)^{2}}(r)$. 
Using the diffeomorphisms $\hat{\phi}_{t, i}$ and $\phi_{i}$ we now extend $\eta_{t}$ to a smooth function on $L_{\boldsymbol{t}}$ by setting $\eta_{t} \equiv 0$ on $\left(\hat{L} \backslash \hat{S}^{*}\right) \cup\left(\Sigma^{*} \times\left[t \hat{R}, t^{a}\right]\right)$ and $\eta_{t} \equiv 1$ on $\left(L \backslash S^{*}\right) \cup$ $\left(\Sigma^{*} \times\left[t^{b}, \epsilon\right]\right)$.

For any $f \in W_{k, \boldsymbol{\beta}_{t}}^{p}$,

$$
\|f\|_{W_{k, \boldsymbol{\beta}_{t}}^{p}} \leq\left\|\eta_{t} f\right\|_{W_{k, \boldsymbol{\beta}_{t}}^{p}}+\left\|\left(1-\eta_{t}\right) f\right\|_{W_{k, \boldsymbol{\beta}_{t}}^{p}} .
$$

Note that $\eta_{t} f$ has support in $\left(\Sigma^{*} \times\left[t^{a}, \epsilon\right]\right) \cup\left(L \backslash S^{*}\right)$, where, up to identifications via the diffeomorphisms $\phi_{i},\left(g_{\boldsymbol{t}}, \rho_{\boldsymbol{t}}\right)=(g, \rho), \boldsymbol{\beta}_{\boldsymbol{t}}=\boldsymbol{\beta}$. Thus

$$
\begin{aligned}
\left\|\eta_{t} f\right\|_{W_{k, \beta_{t}}^{p}\left(g_{t}\right)}= & \left\|\eta_{t} f\right\|_{W_{k, \boldsymbol{\beta}}^{p}(g)} \\
\leq & C_{g}\left\|\Delta_{g}\left(\eta_{t} f\right)\right\|_{W_{k-2, \boldsymbol{\beta}-2}^{p}(g)} \\
= & C_{g}\left\|\Delta_{g_{t}}\left(\eta_{t} f\right)\right\|_{W_{k-2, \boldsymbol{\beta}_{t}-2}^{p}\left(g_{t}\right)} \\
\leq & C_{g}\left(\left\|\eta_{t} \Delta_{g_{t}} f\right\|_{W_{k-2, \beta_{t}-2}^{p}}+\left\|\eta_{t}^{\prime} \nabla f\right\|_{W_{k-2, \boldsymbol{\beta}_{t}-2}^{p}}\right. \\
& \left.+\left\|\eta_{t}^{\prime \prime} f\right\|_{W_{k-2, \boldsymbol{\beta}_{t}-2}^{p}}\right)
\end{aligned}
$$

where we drop unnecessary constants. Applying the Leibniz rule to expressions of the form $\nabla^{j}\left(\eta_{t} \Delta_{g_{t}} f\right)$ we find (again up to constants)

$$
\begin{aligned}
\left\|\eta_{t} \Delta_{g_{t}} f\right\|_{W_{k-2, \boldsymbol{\beta}_{t}-2}^{p}}^{p} & \leq \sum_{j=0}^{k-2} \sum_{l=0}^{j} \int\left|\rho^{l} \nabla^{l} \eta_{t}\right|_{g_{t}}^{p}\left|\rho^{2-\boldsymbol{\beta}_{t}+j-l} \nabla^{j-l} \Delta_{g_{t}} f\right|_{g_{t}}^{p} \rho^{-m_{\operatorname{vol}_{g_{t}}}} \\
& \leq\left(1+\left(\frac{C}{|\log t|}\right)^{p}\right)\left\|\Delta_{g_{t}} f\right\|_{W_{k-2, \boldsymbol{\beta}_{t}-2}^{p}}^{p} .
\end{aligned}
$$

We conclude that

$$
\left\|\eta_{t} \Delta_{g_{t}} f\right\|_{W_{k-2, \boldsymbol{\beta}_{t}-2}^{p}} \leq\left\|\Delta_{g_{t}} f\right\|_{W_{k-2, \boldsymbol{\beta}_{t}-2}^{p}}+\frac{C}{|\log t|}\|f\|_{W_{k, \boldsymbol{\beta}_{t}}^{p}} .
$$

Analogously,

$$
\begin{aligned}
\left\|\eta_{t}^{\prime} \nabla f\right\|_{W_{k-2, \boldsymbol{\beta}_{t}-2}}^{p} & \leq \sum_{j=0}^{k-2} \sum_{l=0}^{j} \int\left|\rho^{1+l} \nabla^{l} \eta_{t}^{\prime}\right|_{g_{t}}^{p}\left|\rho^{1-\boldsymbol{\beta}_{t}+j-l} \nabla^{j-l} \nabla f\right|_{g_{t}}^{p} \rho^{-m} \operatorname{vol}_{g_{t}} \\
& \leq\left(\frac{C}{|\log t|}\right)^{p}\|f\|_{W_{k, \boldsymbol{\beta}_{t}}^{p}}^{p} .
\end{aligned}
$$

Similar calculations apply to $\left\|\eta_{t}^{\prime \prime} f\right\|$, ultimately showing that

$$
\left\|\eta_{t}^{\prime} \nabla f\right\|_{W_{k-2, \beta_{t}-2}^{p}} \leq \frac{C}{|\log t|}\|f\|_{W_{k, \beta_{t}}^{p}}, \quad\left\|\eta_{t}^{\prime \prime} f\right\|_{W_{k-2, \beta_{t}-2}^{p}} \leq \frac{C}{|\log t|}\|f\|_{W_{k, \beta_{t}}^{p}} .
$$


The function $\left(1-\eta_{t}\right) f$ has support in $\left(\hat{L} \backslash \hat{S}^{*}\right) \cup\left(\Sigma^{*} \times\left[t \hat{R}, t^{b}\right]\right)$. On this space Definition 11.6 shows that $\boldsymbol{\beta}_{\boldsymbol{t}}=\hat{\boldsymbol{\beta}}$. Furthermore, on the $i$ th component $\Sigma_{i} \times\left[t \hat{R}, t^{b}\right]$ and up to identifications via the diffeomorphisms $\hat{\phi}_{t, i}$, Lemma 11.9 shows that $g_{\boldsymbol{t}}$ is scaled-equivalent to $t^{2} \hat{g}$ and $\rho_{\boldsymbol{t}}=t \hat{\rho}$.

Using Corollary 6.11 we thus find

$$
\begin{aligned}
\left\|\left(1-\eta_{t}\right) f\right\|_{W_{k, \boldsymbol{\beta}_{t}}^{p}\left(g_{t}, \rho_{t}\right)} & \simeq\left\|\left(1-\eta_{t}\right) f\right\|_{W_{k, \hat{\boldsymbol{\beta}}}^{p}}\left(t^{2} \hat{g}, t \hat{\rho}\right) \\
& =t^{-\beta_{i}}\left\|\left(1-\eta_{t}\right) f\right\|_{W_{k, \hat{\boldsymbol{\beta}}}^{p}(\hat{g}, \hat{\rho})} \\
& \leq t^{-\beta_{i}} C_{\hat{g}}\left\|\Delta_{\hat{g}}\left(\left(1-\eta_{t}\right) f\right)\right\|_{W_{k-2, \hat{\boldsymbol{\beta}}-2}^{p}(\hat{g}, \hat{\rho})} \\
& =t^{2-\beta_{i}} C_{\hat{g}}\left\|\Delta_{t^{2} \hat{g}}\left(\left(1-\eta_{t}\right) f\right)\right\|_{W_{k-2, \hat{\boldsymbol{\beta}}-2}^{p}}(\hat{g}, \hat{\rho}) \\
& =C_{\hat{g}}\left\|\Delta_{t^{2} \hat{g}}\left(\left(1-\eta_{t}\right) f\right)\right\|_{W_{k-2, \hat{\boldsymbol{\beta}}-2}^{p}\left(t^{2} \hat{g}, t \hat{\rho}\right)} \\
& \simeq C_{\hat{g}}\left\|\Delta_{g_{t}}\left(\left(1-\eta_{t}\right) f\right)\right\|_{W_{k-2, \boldsymbol{\beta}_{t-2}}^{p}\left(g_{t}, \rho_{t}\right)},
\end{aligned}
$$

where $\simeq$ replaces multiplicative constants. We now continue as above. Combining the above results leads to an inequality of the form

$$
\|f\|_{W_{k, \beta_{t}}^{p}} \leq\left(C_{g}+C_{\hat{g}}\right)\left(\left\|\Delta_{g_{t}} f\right\|_{W_{k-2, \boldsymbol{\beta}_{t}-2}^{p}}+\frac{C}{|\log t|}\|f\|_{W_{k, \boldsymbol{\beta}_{t}}^{p}}\right) .
$$

For $t$ sufficiently small we can absorb the second term on the right-hand side into the left-hand side, proving the claim.

Smooth compact manifolds. Assume the set $\hat{S}^{* *} \cup S^{* *}$ is empty, so that $\hat{L} \# L$ is smooth and compact. In this case the Laplace operator, acting on functions, always has kernel: the space of constants $\mathbb{R}$. We can thus achieve injectivity only by restricting ourselves to a subspace transverse to constants. Furthermore, if we want the invertibility constant to be independent of $\boldsymbol{t}$ we must allow the subspace to depend on $\boldsymbol{t}$, as follows.

Theorem 12.3. Let $\left(L, g, \rho, S^{*}\right),\left(\hat{L}, \hat{g}, \hat{\rho}, \hat{S}^{*}\right)$ be marked compatible conifolds such that the parametric connect sums $\left(L_{\boldsymbol{t}}, g_{\boldsymbol{t}}, \rho_{\boldsymbol{t}}\right)$ are smooth and compact. Choose constant weights $\boldsymbol{\beta}=\hat{\boldsymbol{\beta}} \in(2-m, 0)$ and define $\boldsymbol{\beta}_{\boldsymbol{t}}$ as usual.

(1) Assume $L$ has only one connected component. Then there exists a constant $C>0$ and, for each $\boldsymbol{t}$ sufficiently small, a subspace $E_{\boldsymbol{t}} \subset$ $W_{k, \boldsymbol{t}}^{p}\left(L_{\boldsymbol{t}}\right)$ such that

$$
W_{k, \boldsymbol{t}}^{p}\left(L_{\boldsymbol{t}}\right)=E_{\boldsymbol{t}} \oplus \mathbb{R}
$$


and, for all $f \in E_{\boldsymbol{t}}$,

$$
\|f\|_{W_{k, \boldsymbol{\beta}_{t}}^{p}} \leq C\left\|\Delta_{g_{t}} f\right\|_{W_{k-2, \boldsymbol{\beta}_{t}-2}^{p}} .
$$

Furthermore, the image of the restricted operator $\Delta_{g_{t} \mid E_{t}}$ coincides with the image of the full operator $\Delta_{g_{t}}$.

(2) Assume $L$ has $k>1$ connected components. Then there exists a constant $C>0$ and, for each $\boldsymbol{t}$ sufficiently small, a codimension $k$ subspace $E_{\boldsymbol{t}} \subset W_{k, \boldsymbol{t}}^{p}\left(L_{\boldsymbol{t}}\right)$ transverse to constants such that, for all $f \in E_{\boldsymbol{t}}$,

$$
\|f\|_{W_{k, \beta_{t}}^{p}} \leq C\left\|\Delta_{g_{t}} f\right\|_{W_{k-2, \beta_{t}-2}^{p}} .
$$

Proof. Assume $L$ has one connected component. Choose any closed subspace $E \subset W_{k, \boldsymbol{\beta}}^{p}(L)$ such that

$$
W_{k, \boldsymbol{\beta}}^{p}(L)=E \oplus \mathbb{R}
$$

Define $\eta_{\boldsymbol{t}}$ as in the proof of Theorem 12.2. Extending it to zero on the CS ends of $L$, we can think of it as an element of $W_{k, \boldsymbol{\beta}}^{p}(L)$. One can check that $\eta_{\boldsymbol{t}} \rightarrow 1$ in the $W_{k, \boldsymbol{\beta}}^{p}$ norm as $\boldsymbol{t} \rightarrow 0$ so, for small $\boldsymbol{t}, \eta_{\boldsymbol{t}} \notin E$. The multiplication map

$$
P_{\boldsymbol{t}}: W_{k, \boldsymbol{\beta}_{\boldsymbol{t}}}^{p}\left(L_{\boldsymbol{t}}\right) \rightarrow W_{k, \boldsymbol{\beta}}^{p}(L), \quad f \mapsto \eta_{\boldsymbol{t}} f,
$$

is linear and uniformly continuous with respect to the parameter $\boldsymbol{t}$, so $E_{\boldsymbol{t}}:=$ $P_{\boldsymbol{t}}^{-1}(E)$ is linear and closed. Since $\eta_{\boldsymbol{t}}$ does not belong to $E$, constants do not belong to $E_{t}$. To confirm that $E_{t}$ has codimension 1, choose any linear function $Q: W_{k, \boldsymbol{\beta}}^{p}(L) \rightarrow \mathbb{R}$ such that $E=\operatorname{Ker}(Q)$. Then $E_{\boldsymbol{t}}=\operatorname{Ker}\left(Q \circ P_{\boldsymbol{t}}\right)$, so it is defined by one linear condition. This proves Decomposition 12.1.

Consider $\Delta_{g_{t}}$ restricted to $E_{\boldsymbol{t}}$. It is clearly injective. One can check that it is uniformly injective exactly as in Theorem 12.2.

Now assume $L$ has multiple components $L_{1}, \ldots, L_{k}$. For each $L_{i}$, choose a closed subspace $E_{i} \subset W_{k, \boldsymbol{\beta}}^{p}\left(L_{i}\right)$ as above. The multiplication map

$$
W_{k, \boldsymbol{\beta}_{t}}^{p}\left(L_{\boldsymbol{t}}\right) \rightarrow \bigoplus W_{k, \boldsymbol{\beta}_{t}}^{p}\left(L_{i}\right), \quad f \mapsto \eta_{\boldsymbol{t}} f
$$

is again linear and uniformly continuous, so we can define $E_{\boldsymbol{t}}$ as the inverse of $E_{1} \oplus \ldots, \oplus E_{k}$. One can again check that it has codimension $k$ and that, restricted to this space, $\Delta_{g_{t}}$ is uniformly injective.

Remark 12.4. Note that, even though $L_{\boldsymbol{t}}$ is smooth and compact, the proof of Theorem 12.3 requires the use of radius functions and weights on the necks. 


\section{Further Sobolev-type inequalities on conifold connect sums}

Given a conifold $(L, g)$, we can also apply the theory of Section 9 to the elliptic operator

$$
D_{g}=d \oplus d_{g}^{*}: W_{k, \boldsymbol{\beta}}^{p}\left(\Lambda^{\text {even }}\right) \rightarrow W_{k-1, \boldsymbol{\beta}-1}^{p}\left(\Lambda^{\text {odd }}\right),
$$

defined from the bundle of all even-dimensional forms on $L$ to the bundle of all odd-dimensional forms. As for the Laplacian, it is possible to define and study the exceptional weights for this operator. For any non-exceptional weight $\boldsymbol{\beta}$, the operator $D_{g}$ of Equation (13.1) is Fredholm. This implies that

$$
D_{g}: W_{k, \boldsymbol{\beta}}^{p}\left(\Lambda^{\mathrm{even}}\right) / \operatorname{Ker}\left(D_{g}\right) \rightarrow W_{k-1, \boldsymbol{\beta}-1}^{p}\left(\Lambda^{\text {odd }}\right)
$$

is a topological isomorphism onto its image. Note that $W_{k, \boldsymbol{\beta}}^{p}(L) / \operatorname{Ker}\left(D_{g}\right)$ is closed in $W_{k, \boldsymbol{\beta}}^{p}\left(\Lambda^{\text {even }}\right) / \operatorname{Ker}\left(D_{g}\right)$. It follows that $d\left(W_{k, \boldsymbol{\beta}}^{p}(L)\right)=D_{g}\left(W_{k, \boldsymbol{\beta}}^{p}(L)\right)=$ $D_{g}\left(W_{k, \boldsymbol{\beta}}^{p}(L) / \operatorname{Ker}\left(D_{g}\right)\right)$ is closed in $\operatorname{Im}\left(D_{g}\right)$, thus in $W_{k-1, \boldsymbol{\beta}-1}^{p}\left(\Lambda^{\text {odd }}\right)$. We can conclude that the restricted operator

$$
d: W_{k, \boldsymbol{\beta}}^{p}(L) \rightarrow W_{k-1, \boldsymbol{\beta}-1}^{p}\left(\Lambda^{1}\right)
$$

has closed image. Note that $\operatorname{Ker}(d)$ can only contain constants. If the choice of weights is such that constants do not belong to the space $W_{k, \boldsymbol{\beta}}^{p}(L)$, the operator $d$ of Equation (13.2) is a topological isomorphism onto its image and can be inverted. In particular there exists $C>0$ such that, for any $f \in W_{k, \boldsymbol{\beta}}^{p}(L)$,

$$
\|f\|_{W_{k, \boldsymbol{\beta}}^{p}} \leq C\|d f\|_{W_{k-1, \boldsymbol{\beta}-1}^{p}} .
$$

We now want to show that, on conifolds obtained as parametric connect sums, such $C$ can chosen independently of $\boldsymbol{t}$. For brevity, we restrict our attention to the non-compact case.

Theorem 13.1. Let $\left(\hat{L}, \hat{g}, \hat{\rho}, \hat{\boldsymbol{\beta}}, \hat{S}^{*}\right)$ be a weighted AC-marked conifold. Assume that $\hat{\boldsymbol{\beta}}$ is non-exceptional for the operator

$$
D_{\hat{g}}: W_{k, \hat{\boldsymbol{\beta}}}^{p}\left(\Lambda^{\text {even }}\right) \rightarrow W_{k-1, \hat{\boldsymbol{\beta}}-1}^{p}\left(\Lambda^{\text {odd }}\right)
$$

defined on the manifold $\hat{L}$ and that $\hat{\beta}_{i}<0$ for all ends $\hat{S}_{i} \in \hat{S}^{*}$. 
Let $\left(L, g, \rho, \boldsymbol{\beta}, S^{*}\right)$ be a weighted CS-marked conifold. Assume $\boldsymbol{\beta}$ is nonexceptional for the operator

$$
D_{g}: W_{k, \boldsymbol{\beta}}^{p}\left(\Lambda^{\text {even }}\right) \rightarrow W_{k-1, \boldsymbol{\beta}-1}^{p}\left(\Lambda^{\text {odd }}\right)
$$

defined on the manifold $L$ and that each connected component of $L$ has at least one end, e.g., $S^{\prime}$, satisfying the condition

$$
\left\{\begin{array}{l}
\beta^{\prime}<0, \text { if } S^{\prime} \text { is } A C \\
\beta^{\prime}>0, \text { if } S^{\prime} \text { is } C S .
\end{array}\right.
$$

Now assume that $L, \hat{L}$ are compatible. Then, for all ends $S_{i} \in S^{*}, \beta_{i}=\hat{\beta}_{i}<$ 0. This implies that $S^{\prime} \in S^{* *}$ so each connect sum $L_{\boldsymbol{t}}$ has at least one end.

There exists $C>0$ such that, for all $f \in W_{k, \boldsymbol{\beta}_{t}}^{p}\left(L_{\boldsymbol{t}}\right)$,

$$
\|f\|_{W_{k, \boldsymbol{\beta}_{t}}^{p}} \leq C\|d f\|_{W_{k-1, \beta_{t}-1}^{p}} .
$$

Proof. As seen above, the assumptions prove that the operator $d$ is a topological isomorphism (onto its image) between Sobolev spaces on both manifolds $L, \hat{L}$. This means that there exist constants $C_{g}, C_{\hat{g}}$ satisfying the analogue of Equation (13.3) on both manifolds separately. We can use $C_{g}, C_{\hat{g}}$ to build $C$ satisfying Equation (13.3) on $L_{\boldsymbol{t}}$ using the same ideas introduced in the proof of Theorem 12.2. There is only one difference, as follows. In the proof of Theorem 12.2 we use the equality

$$
t^{-\beta_{i}} C_{\hat{g}}\left\|\Delta_{\hat{g}}\left(\left(1-\eta_{t}\right) f\right)\right\|_{W_{k-2, \hat{\boldsymbol{\beta}}-2}^{p}(\hat{g}, \hat{\rho})}=t^{2-\beta_{i}} C_{\hat{g}}\left\|\Delta_{t^{2} \hat{g}}\left(\left(1-\eta_{t}\right) f\right)\right\|_{W_{k-2, \hat{\boldsymbol{\beta}}-2}^{p}(\hat{g}, \hat{\rho})} \cdot
$$

The factor $t^{2-\beta_{i}}$ is then cancelled by rescaling. In particular, the above equality uses the fact that the Laplacian depends on the metric and rescales in a specific way.

In the case at hand the operator $d$ does not depend on the metric. However, note that it takes functions into 1-forms: it is this property that allows us to conclude. Specifically, setting $\alpha_{t}=d\left(\left(1-\eta_{t}\right) f\right)$ and assuming $\hat{\boldsymbol{\beta}}$ is constant to simplify the notation, we find:

$$
\begin{aligned}
\left\|\alpha_{t}\right\|_{W_{k-1, \hat{\boldsymbol{\beta}}-1}^{p}(\hat{g}, \hat{\rho})}^{p} & =\sum_{j} \int_{\hat{L}}\left|\hat{\rho}^{1-\hat{\boldsymbol{\beta}}+j} \nabla^{j} \alpha_{t}\right|_{\hat{g} \otimes \hat{g}}^{p} \hat{\rho}^{-m} \operatorname{vol}_{\hat{g}} \\
& =t^{p \hat{\boldsymbol{\beta}}} \sum_{j} \int_{\hat{L}}\left|(t \hat{\rho})^{1-\hat{\boldsymbol{\beta}}+j} \nabla^{j} \alpha_{t}\right|_{t^{2} \hat{g} \otimes t^{2} \hat{g}}^{p}(t \hat{\rho})^{-m} \operatorname{vol}_{t^{2} \hat{g}} \\
& =t^{p \hat{\boldsymbol{\beta}}}\left\|\alpha_{t}\right\|_{W_{k-1, \hat{\boldsymbol{\beta}}-1}^{p}\left(t^{2} \hat{g}, t \hat{\rho}\right)^{p}}^{p}
\end{aligned}
$$

The proof can now continue as for Theorem 12.2. 
Combining Theorems 11.7 and 13.1, we obtain the following improvement of the weighted Sobolev Embedding Theorems, Part 1, for parametric connect sums.

Corollary 13.2. Let $\left(L, g, \rho, \boldsymbol{\beta}, S^{*}\right),\left(\hat{L}, \hat{g}, \hat{\rho}, \hat{\boldsymbol{\beta}}, \hat{S}^{*}\right)$ be marked compatible conifolds as in Theorem 13.1. Define $L_{\boldsymbol{t}}$ as in Definition 11.6. Then there exists $C>0$ such that, for all $1 \leq p<m$, $\boldsymbol{t}$ and $f \in W_{1, \boldsymbol{\beta}_{\boldsymbol{t}}}^{p}\left(L_{\boldsymbol{t}}\right)$,

$$
\|f\|_{L_{\boldsymbol{\beta}_{\boldsymbol{t}}}^{p^{*}}} \leq C\|d f\|_{L_{\boldsymbol{\beta}_{\boldsymbol{t}}-1}^{p}} .
$$

Remark 13.3. Following standard terminology in the literature we can refer to Equation (13.3) as a "uniform weighted Poincaré inequality" and to Corollary 13.2 as a "uniform weighted Gagliardo-Nirenberg-Sobolev inequality". Alternatively, following [4] Chapter 8, the latter is a "uniform weighted Euclidean-type Sobolev inequality".

\section{Acknowledgments}

I would like to thank D. Joyce for many useful suggestions and discussions concerning the material of this paper. I also thank M. Haskins and J. Lotay for several conversations. Part of this work was carried out while I was a Marie Curie EIF Fellow at the University of Oxford. It has also been supported by a Marie Curie ERG grant at the Scuola Normale Superiore in Pisa.

\section{References}

[1] R. A. Adams, Sobolev spaces, Pure and Applied Math. Vol. 65, Academic Press, New York-London, 1975.

[2] C. Arezzo and Frank Pacard, Complete, embedded, minimal ndimensional submanifolds in $\mathbb{C}^{n}$, Comm. Pure Appl. Math. 56 (3) (2003), 283-327.

[3] A. Butscher, Regularizing a singular special Lagrangian variety, Comm. Anal. Geom. 12 (4) (2004), 733-791.

[4] E. Hebey, Nonlinear analysis on manifolds: Sobolev spaces and inequalities, Courant Lecture Notes in Math. vol. 5, New York University Courant Institute of Mathematical Sciences, New York, 1999.

[5] D. Joyce, Special Lagrangian submanifolds with isolated conical singularities. I. Regularity, Ann. Global Anal. Geom. 25 (3)(2004), 201-251. 
[6] _ Special Lagrangian submanifolds with isolated conical singularities. III. Desingularization, the unobstructed case, Ann. Global Anal. Geom. 26 (1) (2004), 1-58.

[7] _ Special Lagrangian submanifolds with isolated conical singularities. IV. Desingularization, obstructions and families, Ann. Global Anal. Geom. 26 (2) (2004), 117-174.

[8] D. Joyce and S. Salur, Deformations of asymptotically cylindrical coassociative submanifolds with fixed boundary, Geom. Topol. 9 (2005), 1115-1146 (electronic).

[9] Y.-I. Lee, Embedded special Lagrangian submanifolds in Calabi-- Yau manifolds, Comm. Anal. Geom. 11 (3) (2003), 391-423.

[10] R. Lockhart, Fredholm, Hodge and Liouville theorems on noncompact manifolds, Trans. Amer. Math. Soc. 301 (1) (1987), 1-35.

[11] R. Lockhart and R. C. McOwen, Elliptic differential operators on noncompact manifolds, Ann. Scuola Norm. Sup. Pisa Cl. Sci. (4) 12 (3) (1985), 409-447.

[12] J. D. Lotay, Desingularization of coassociative 4-folds with conical singularities, Geom. Funct. Anal. 18 (6)(2009), 2055-2100.

[13] R. B. Melrose, The Atiyah-Patodi-Singer index theorem, Research Notes in Math., vol. 4, A K Peters Ltd., Wellesley, MA, 1993.

[14] T. Pacini, Special Lagrangian conifolds, I: Moduli spaces, Proc. LMS, to appear, doi:10.1112/plms/pds093.

[15] _ Special Lagrangian conifolds, II: Gluing constructions in $\mathbb{C}^{m}$, Proc. LMS, to appear, doi:10.1112/plms/pds092.

Scuola Normale Superiore, Piazza dei Cavalieri 7, 56126 Pisa, Italy

E-mail address: tommaso.pacini@sns.it

RECEIVED MARCH 12, 2012 